\title{
TITLE: Molecular Accessibility in Oxidized and Dried Coals
}

\author{
FINAL REPORT
}

REPORT PERIOD: 9/1/93 - 8/31/97

PRINCIPAL INVESTIGATOR: Lowell D. Kispert, Department of Chemistry The University of Alabama Tuscaloosa, AL 35487-0336

ISSUED：September 1997

REVISED: July 1999

DOE AWARD NO.: DE-FG22-93PC93202--16

SUBMITTING ORGANIZATION:

Chemistry Department, Box 870336

University of Alabama

Tuscaloosa, AL 35487 


\section{DISCLAIMER}

This report was prepared as an account of work sponsored by an agency of the United States Government. Neither the United States Government nor any agency thereof, nor any of their employees, makes any warranty, express or implied, or assumes any legal liability or responsibility for the accuracy, completeness, or usefulness of any information, apparatus, product, or process disclosed, or represents that its use would not infringe privately owned rights. Reference herein to any specific commercial product, process, or service by trade name, trademark, manufacturer, or otherwise does not necessarily constitute or imply its endorsement, recommendation, or favoring by the United States Government or any agency thereof. The views and opinions of authors expressed herein do not necessarily state or reflect those of the United States Government or any agency thereof. 


\begin{abstract}
Changes in physical and chemical structure of the micropore system in eight solvent swelled Argonne Premium Coal Sample (APCS) coals upon weathering were studied using the EPR spin probe method. Spin probes, which are allowed to diffuse into the coal structure during swelling, are trapped when the swelling solvent is removed. Excess spin probes are removed from the coal surface and larger pores so that only the presence of spin probes trapped in pores which closely approximate the size of the spin probe are detected. Detailed explanations and illustrations of the experimental procedure used are given.

Careful examination of the weathering process on coal as a function of rank was accomplished using the EPR spin probe method. The retention of spin probes in eight APCS coals provided valuable insight into both the loss of water and the oxidation which occur during the weathering process. The results could be explained in terms of the autoxidation process observed in other polymeric systems. It was shown that initial oxidation of coal can result in increased cross-linking in the coal structure. As the oxidation process continued, both the covalent and hydrogen bonded character of the coal were significantly altered. The retention character of some coals during oxidation was shown to change by as much as three orders of magnitude. Experiments were performed to study the effects of short term oxidation and dehydration on coal structure by exposing the coal samples to argon or oxygen for time periods up to five minutes. The results indicate that the structure of coal is extremely sensitive to environmental changes and exhibits significant changes in as little as 30 seconds. Exposure of Illinois \#6 coal to argon or oxygen for 30 seconds caused a decrease in the retention of polar spin probes by as much as an order of magnitude.
\end{abstract}

The studies presented here suggest that the structure of coal is dynamic in nature, and has an intimate relationship with the nature of its environment. This method has been shown to be very sensitive to structural changes brought about in coal by oxidation and dehydration, and can be used to follow changes in coal during the swelling process. Additional ideas for future studies using the EPR spin probe method are also discussed. 


\section{TABLE OF CONTENTS}

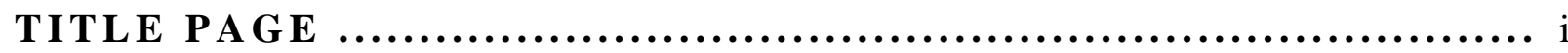

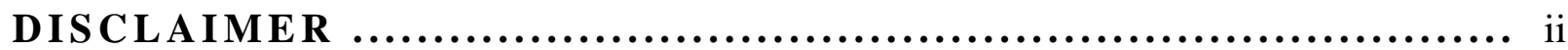

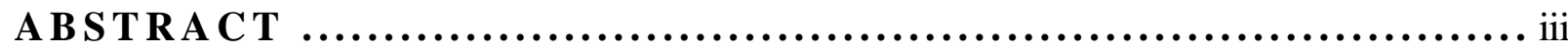

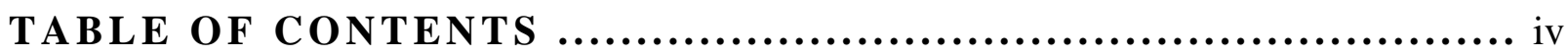

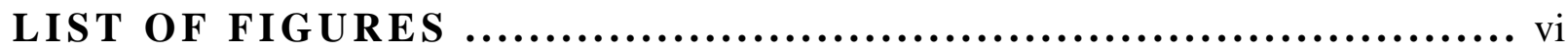

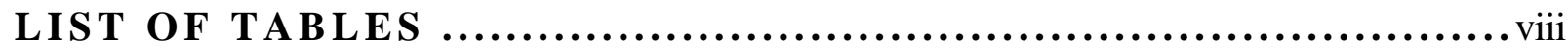

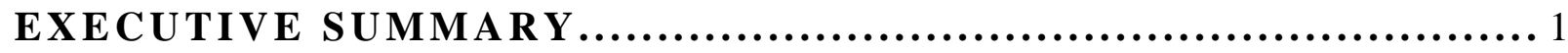

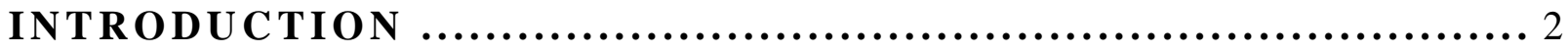

The Argonne Premium Coal Sample Program …........................................ 2

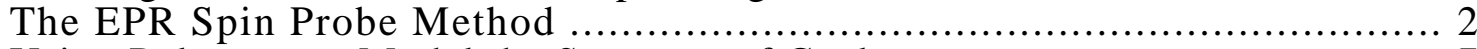

Using Polymers to Model the Structure of Coal …….................................. 7

The Weathering Process and Coal................................................... 8

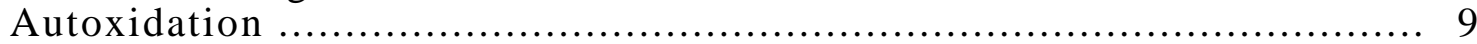

Methods for Studying the Structure of Coal ……................................. 13

SANS, SAXS and Light Scattering .................................................. 13

SEM and Imaging Techniques ................................................... 14

NMR Spin Lattice Relaxation and Proton NMR ….................................. 14

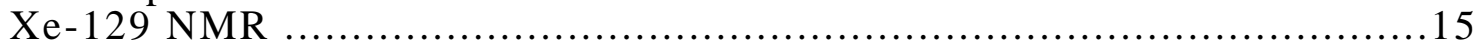

EPR Spin Probe Method …..................................................... 15

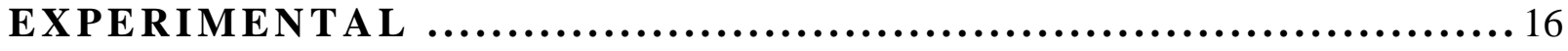

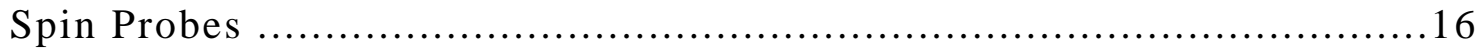

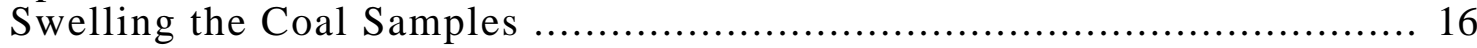

Determining Spin Concentrations ……............................................ 18

Binary Swelling Solvent Systems …............................................. 19

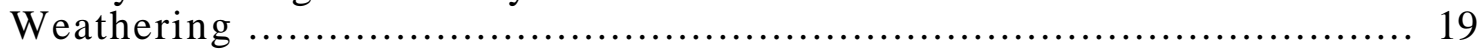

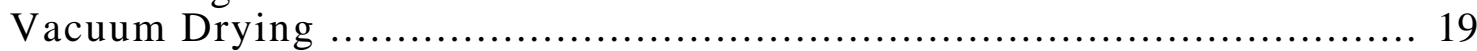

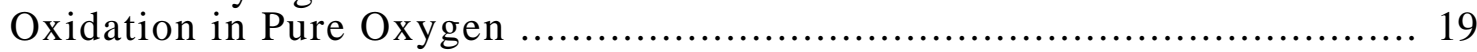

Short Term Oxidation and Dehydration ……....................................... 20

High Pressure Swelling ....................................................... 21

Surface Area Determination and Errors ……..................................... 22

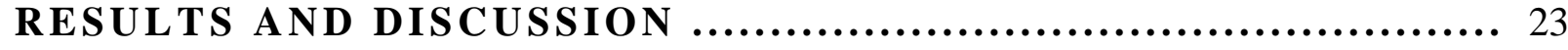

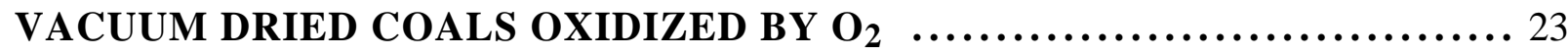

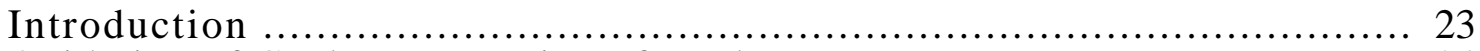

Oxidation of Coal as a Function of Rank ......................................... 24

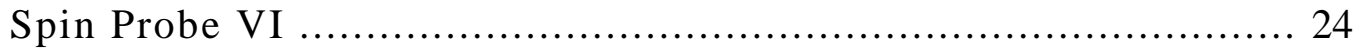

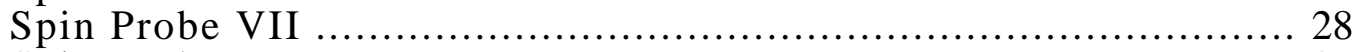

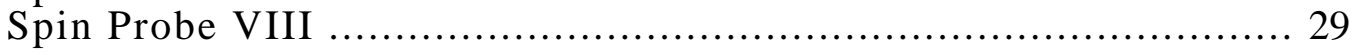


Oxidation Of Individual Vacuum Dried Coals

Beulah Zap Lignite (74.1\% carbon dmmf) ……............................... 31

Wyodak Anderson Caol \& 76.0\% carbon dmmf) ................................ 33

Illinois \#6 Coal (80.7\% carbon dmmf) ........................................ 36

Blind Canyon Coal (81.3\% carbon dmmf) ……............................ 38

Lewiston-Stockton Coal (85.0\% carbon dmmf) ................................. 40

Pittsburgh \#8 Coal (85.5\% carbon dmmf) ...................................... 43

Upper Freport Coal (88.1\% carbon dmmf) .................................... 45

Poccahontas \#3 Coal (91.8\% carbon dmmf) ................................... 47

Summary of the Oxidation Process in Vacuum Dried Coals .............................. 49

Spin Probe VIII ....................................................... 50

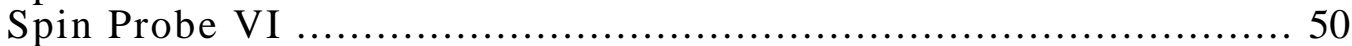

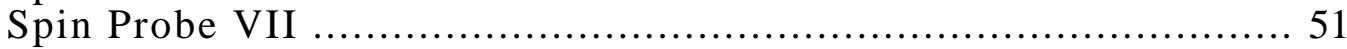

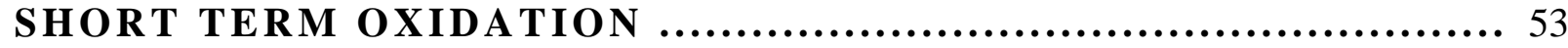

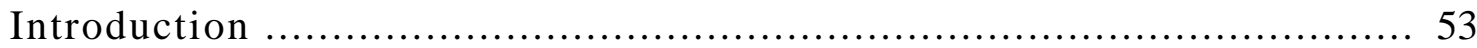

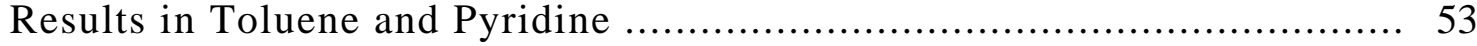

BINARY SWELLING SOLVENT SYSTEMS ............................ 57

Introduction ...................................................................... 57

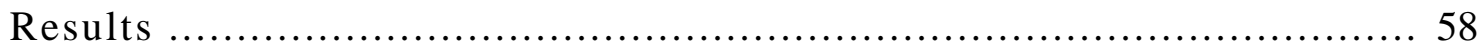

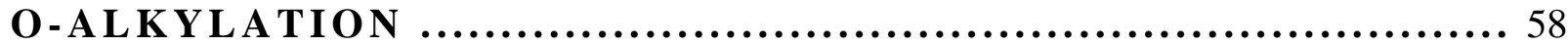

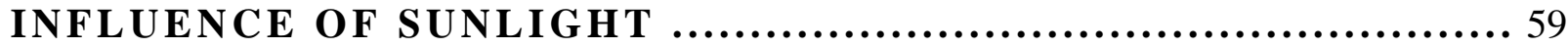

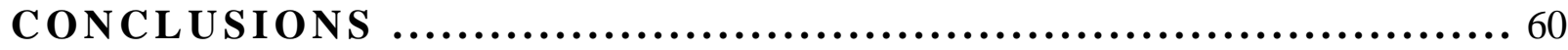

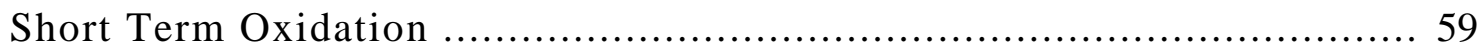

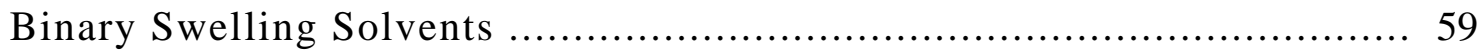

RECOMMENDATIONS： FUTURE WORK ............................. 61

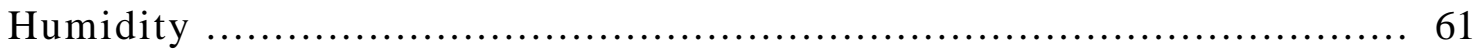

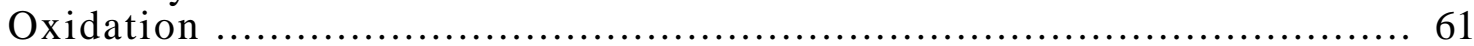

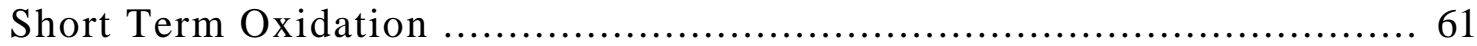

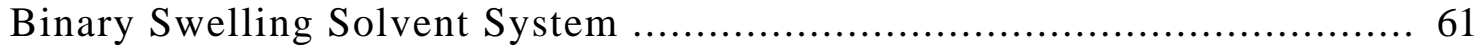

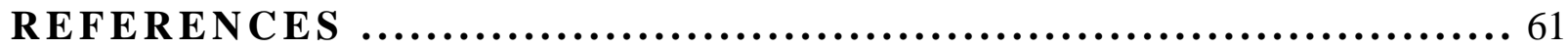

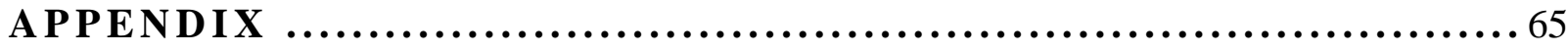




\section{LIST OF FIGURES}

Figure 1. The general structure of a nitroxyl free radical.

Figure 2. A systematic of the coal structure during swelling and the diffusion of the spin probes during this process.

Figure 3. Illustration of the coal structure after the swelling solvent has been removed.

Figure 4. A diagram of the structure of the coal after the remaining spin probes have been washed off with cyclohexane.

Figure 5. A cartoon of the cross-link structure in coal.

Figure 6. The hydrogen bond interactions of spn probes I, VI and VII with the hydroxyl groups in coal structure.

Figure 7. An illustration of a coal microchannel with radical sites.

Figure 8. The formation of hydroperoxide radicals in the coal structure.

Figure 9. The formation of peroxide cross-links and the relocation of radical sites from hydroperoxide radicals.

Figure 10. The formation of ether linkages in coal from peroxide cross-links and the formation of other covalent cross-links by combination of radical sites.

Figure 11. Spin Probes I, VI, VII, VII and XIII.

Figure 12. The basic swelling procedure for the inclusion of spin probes in APCS coals swelled under ambient pressure and temperature

Figure 13. The procedure for preparing the Illinois \#6 coal for short term oxidation and dehydration.

Figure 14. The procedure used for high pressure swelling studies.

Figure 15. The retention of spin probe VI in $\mathrm{O}_{2}$ oxidized coals swelled in toluene, expressed as a function of both rank (percent carbon) and exposure period (days of exposure to oxygen) up to an exposure period of 64 days.

Figure 16. The retention of spin probe VI in $\mathrm{O}_{2}$ oxidized coals swelled in toluene, expressed as a function of both rank (percent carbon) and exposure period (days of exposure to oxygen) for up to 36 days

Figure 17. The retention of spin probe VI in vacuum dried coals weathered in air for up to 35 days, expressed as a function of weathering period in days of exposure, and coal rank as percent carbon. 
Figure 18. The retention of spin probe VI in fresh coals weathered in air for up to 36 days, expressed as a function of weathering period in days of exposure, and coal rank as percent carbon.

Figure 19. The retention of spin probe VI in fresh coals weathered in air for up to 64 days, expressed as a function of weathering period in days of exposure, and coal rank as percent carbon.

Figure 20. Retention of spin probe VII in vacuum dried APCS coals, oxidized in pure oxygen and swelled in toluene expressed in spins per gram versus days of exposure to oxygen and \% carbon (dmmf).

Figure 21. Retention of spin probe VIII in vacuum dried APCS coals, oxidized in pure oxygen and swelled in toluene, expressed in spins per gram versus days of exposure to oxygen and \% carbon (dmmf).

Figure 22. A comparison of the retention of spin probe VI in coals weathered in air (on the left) and coals oxidized in $\mathrm{O}_{2}$ (on the right). Toluene was used as the swelling solvent.

Figure 23. The retention of spin probes VI (O), VII $(\square)$ and VII $(\Delta)$, - in vacuum dried Beulah Zap lignite swelled in toluene (open symbols) and pyridine (closed symbols) as a function of oxidation period expressed in days.

Figure 24. The retention of spin probes VI (O), VII $(\square)$ and VIII $(\Delta)$ in vacuum dried Wyodak Anderson coal swelled in toluene (open symbols) and pyridine (closed symbols) as a function of oxidation period expressed in days.

Figure 25. The retention of spin probes VI (O), VII $(\square)$ and VIII $(\Delta)$ in vaccum dried Illinois \#6 coal swelled in toluene (open symbols) and pyridine (closed symbols) as a function of oxidation.

Figure 26. The retention of spin probes VI (O), VII ( $\square)$ and VIII $(\Delta)$ in vaccum dried Blind Canyon coal swelled in toluene (open symbols) and pyridine (closed symbols) as a function of oxidation period expressed in days.

Figure 27. The retention of spin probes VI (O), VII $(\square)$ and VIII $(\Delta)$ in vaccum dried Lewiston-Stockton coal swelled in toluene (open symbols) and pyridine (closed symbols) as a function of oxidation period expressed in days.

Figure 28. The retention of spin probes VI in vaccum dried Pittsburgh \#8 coal swelled in toluene as a function of oxidation period expressed in days

Figure 29. The retention of spin probes VI and VIII in vacuum dried Upper Freeport coal swelled in toluene (open symbols) and pyridne (closed symbols) as a function of oxidation period expresssed in days. 
Figure 30. The retention of spin probes VI in vacuum dried Poccahontas \#3 coal swelled in toluene (open symbols) and pyridine (closed symbols) as a function of oxidation period expressed in days.

Figure 31. The retention of spin probe VI in toluene swelled Illinois \#6 coal exposed to both argon argon $(\square)$ and oxygen $(\mathrm{O})$.

Figure 32. The retention of spin probe VII in toluene swelled Illinois \#6 coal exposed to both argon argon ( $\square)$ and oxygen $(\mathrm{O})$.

Figure 33. The retention of spin probe VIII in toluene swelled Illinois \#6 coal exposed to both argon argon $(\square)$ and oxygen $(\mathrm{O})$.

Figure 34. The retention of spin probe VI in pyridine swelled Illinois \#6 coal exposed to both argon $(\square)$ and oxygen $(\mathrm{O})$.

Figure 35. The retention of spin probe VII in pyridine swelled Ilinois \#6 coal exposed to both argon $(\square)$ and oxygen $(\mathrm{O})$.

Figure 36. The retention of spin probe VIII in pyridine swelled Illinois \#6 coal exposed to both argon ( $\square)$ and oxygen (O)

\section{LIST OF TABLES}

Table 1. The chemical character of the APCS coals arranged in order of increasing rank.

Table 2. The experimental operating conditions of the EPR spectrometer.

Table 3. The maximum error in the precision of spin probe concentration measurements. 


\section{EXECUTIVE SUMMARY}

Changes in physical and chemical structure of the micropore system in eight solvent swelled Argonne Premium Coal Sample (APCS) coals upon weathering were studied using the EPR spin probe method. The retention of spin probes in eight APCS coals provided valuable insight into both the loss of water and the oxidation which occur during the weathering process. The results could be explained in terms of the autoxidation process observed in polymeric systems. It was shown that initial oxidation of coal can result in increased cross-linking in the coal structure. Experiments performed to study the effects of short term oxidation and dehydration on coal structure indicated that the structure of coal is extremely sensitive to environmental changes and exhibits significant changes in as little as 30 seconds. It was shown that the addition of pyridine in amounts as small as $100 \mathrm{ppm}$ to toluene in binary swelling solvent experiments could significantly alter the retention of spin probes in the coal structure by as much as an order of magnitude.

The presented studies suggested that the structure of coal is dynamic in nature, and has an intimate relationship with the nature of its environment. This method has been shown to be very sensitive to structural changes brought about in coal. Additional ideas for future studies using the EPR spin probe method are also discussed. 


\section{INTRODUCTION}

\section{The Argonne Premium Coal Sample Program}

Because coal samples ${ }^{1}$ can vary greatly in chemical and physical properties from seam to seam, and even within the same seam, results obtained from different labs around the country cannot be reasonably compared to one another. Only very limited conclusions about the general coal structure can be reached. Any researcher studying the structural properties of a coal must first characterize the coal sample being used. The need for a more standardized coal sample program was addressed at the Coal Sample Bank Workshop held March 27 and 28, 1981 in Atlanta, Georgia. The goal was to establish a bank of premium coal samples for the coal research community which would be "chemically and physically as identical as possible." It was decided to limit the number of coals to be studied to eight coals of various ranks from around the country. Each coal was mined, transported, processed into particle and sample sizes, mixed thoroughly, packaged in humid nitrogen environments and carefully characterized by various techniques. The eight coals chosen for the Argonne Premium Coal Sample program ${ }^{2}$ are described in Table 1.

Table 1. The chemical character of the APCS coals arranged in order of increasing rank.

$\begin{array}{lllllllr}\text { Coal } & \text { State } & \text { Rank } & \text { C } & \text { H } & \text { O } & \text { S } & \text { Ash } \\ & & & & & & & \\ \text { Beulah-Zap } & \text { ND } & \text { Lignite } & 74.1 & 4.9 & 19.1 & 0.8 & 9.7 \\ \text { Wyodak-Anderson } & \text { WY } & \text { Subbituminous } & 76 & 5.4 & 16.9 & 0.63 & 8.8 \\ \text { Illinois \#6 } & \text { IL } & \text { High Vol. Bituminous } & 80.7 & 5.2 & 10.1 & 4.83 & 15.5 \\ \text { Blind Canyon } & \text { UT } & \text { High Vol. Bituminous } & 81.3 & 5.8 & 10.9 & 0.62 & 4.7 \\ \text { Pittsburgh \#8 } & \text { PA } & \text { High Vol. Bituminous } & 85 & 5.4 & 6.9 & 2.19 & 9.3 \\ \text { Lewiston-Stockton } & \text { WV } & \text { High Vol. Bituminous } & 85.5 & 5.4 & 6.7 & 0.71 & 19.8 \\ \text { Upper Freeport } & \text { PA } & \text { Med. Vol. Bituminous } & 88.1 & 4.8 & 4.7 & 2.32 & 13.2 \\ \text { Pocahontas \#3 } & \text { VA } & \text { Low Vol. Bituminous } & 91.8 & 4.5 & 1.7 & 0.66 & 4.8\end{array}$

Although coals are generally characterized by the carbon, hydrogen and oxygen content, it can be seen from the previous data that coals similar in rank can have strongly different inorganic character. This is an important point because coal pairs like Illinois \#6 and Blind Canyon or Pittsburgh \#8 and Lewiston-Stockton are very similar in terms of their carbon, hydrogen and oxygen content. However, the percentage of ash and sulfur in these coals vary greatly. Likewise, the chemical character of these coals can be as equally varied, especially as determined by the EPR spin probe technique used in this study.

\section{The EPR Spin Probe Method}

The molecular accessibility of catalysts in a coal structure is an important consideration in conversion of coal to chemicals. It is the goal of this research to study the process of molecular intercalation in a coal structure during solvent swelling. This is accomplished by using molecular probes which are soluble in a swelling solvent and which can be monitored spectroscopically. Nitroxyl stable free radicals are used as probes so that Electron Paramagnetic Resonance spectroscopy (EPR) can be used to me asure the number of probes trapped in the coal structure. These spin probes can be substituted with various functionalities so that chemical characteristics of the pores in the structure of coal can be studied. By swelling the coal in a spin probe swelling solvent solution, changes in the coal structure can be monitored during a chemical process like oxidation or weathering. 
This technique has been very useful in studying molecular accessibility in coal samples. ${ }^{3-9}$ Use of spin probes has been shown to be advantageous when the spin probes contain polar substituents.4,5 Lignite and subbituminous coal contain carboxyl and hydroxyl groups. Nitrogen functionalities make only minor contributions in coal due to the low nitrogen content in coal. ${ }^{9}$ A spin probe with an amino substituent is a strong hydrogen bond acceptor, capable of only weak hydrogen bond donation. A spin probe with a carboxyl group is a strong hydrogen bond donor, and is only capable of acting as an acceptor through the carbonyl group. Thus these spin probes can interact and detect the presence of hydroxyl and carbonyl groups present in the coal matrix. The effect of size incorporation in the coal matrix can be determined by comparing the retention of a spin probe without a substituent (substituted only with a proton) to one similar in size containing a substituent. Solvents like pyridine are capable of hydrogen bonding with coal. Pyridine has been shown to break up the hydrogen bonding cross-links in the coal structure. ${ }^{10}$ It is possible to displace hydrogen bonded spin probes with pyridine.

The size or molecular volume of the guest molecule will closely approximate the size of the pore in which it is trapped. For the studies included here, the guest molecules are generally limited to sizes which study the micropore system (pores with a diameter of less than 20 angstroms).

The use of stable free radicals as guest molecules was first developed by Silbernagel et. al. for studying the porosity of membranes by EPR methods. ${ }^{11}$ These radicals, whose general structure is given below in Figure 1, have a nitrogen singly bound to oxygen and have four methyl groups on the alpha ring positions to sterically stabilize the radical. The unpaired electron density resides $40 \%$ on nitrogen and $60 \%$ on oxygen. The nitroxyl group (NO) has little effect on the reactivity of any molecule to which it is attached. Consequently, nitroxides are very stable compounds, even at room temperature. The R group represents various functionalities which may be attached to the nitroxyl. ${ }^{1}$

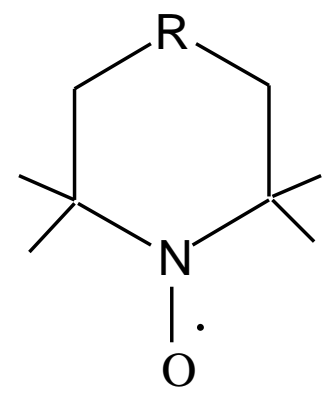

Figure 1. The general structure of a nitroxyl free radical.

Silbernagel's method has been further developed and considerably expanded in our laboratory. The use of the EPR spin probes has proved to be a very valuable technique in gathering information about molecular inclusion and intercalation in coals, coal micropore structure and surface chemistry..$^{3-5,11-15}$ Our results show that swelling is a function of solvent polarity. Coal contains spherical shaped pores which disappear upon swelling. With polar solvents like pyridine, the pores elongate in a direction parallel to the bedding plane. Pore elongation takes place by gradual disruption of hydrogen bonds perpendicular to the bedding plane, resulting in an unzipping of the coal macromolecular structure. Small increases in temperature did not greatly affect the swelling ability of toluene, unlike polar solvents such as pyridine and nitrobenzene, which are strong swelling solvents at higher temperatures. Coal swelling decreases with rank, due to both decrease in porosity and in 
the hydrogen bond cross-link density. This information provided insight into the hydrogen bond cross-linking in coal and the effects of swelling solvents on these cross-links.

To accomplish the intercalation of the EPR spin probes in the coal structure, these probe molecules are dissolved in the swelling solvent. The coal is then covered with the solution and agitated for several hours. The guest molecules can then diffuse into the opened coal structure as shown in Figure 2.

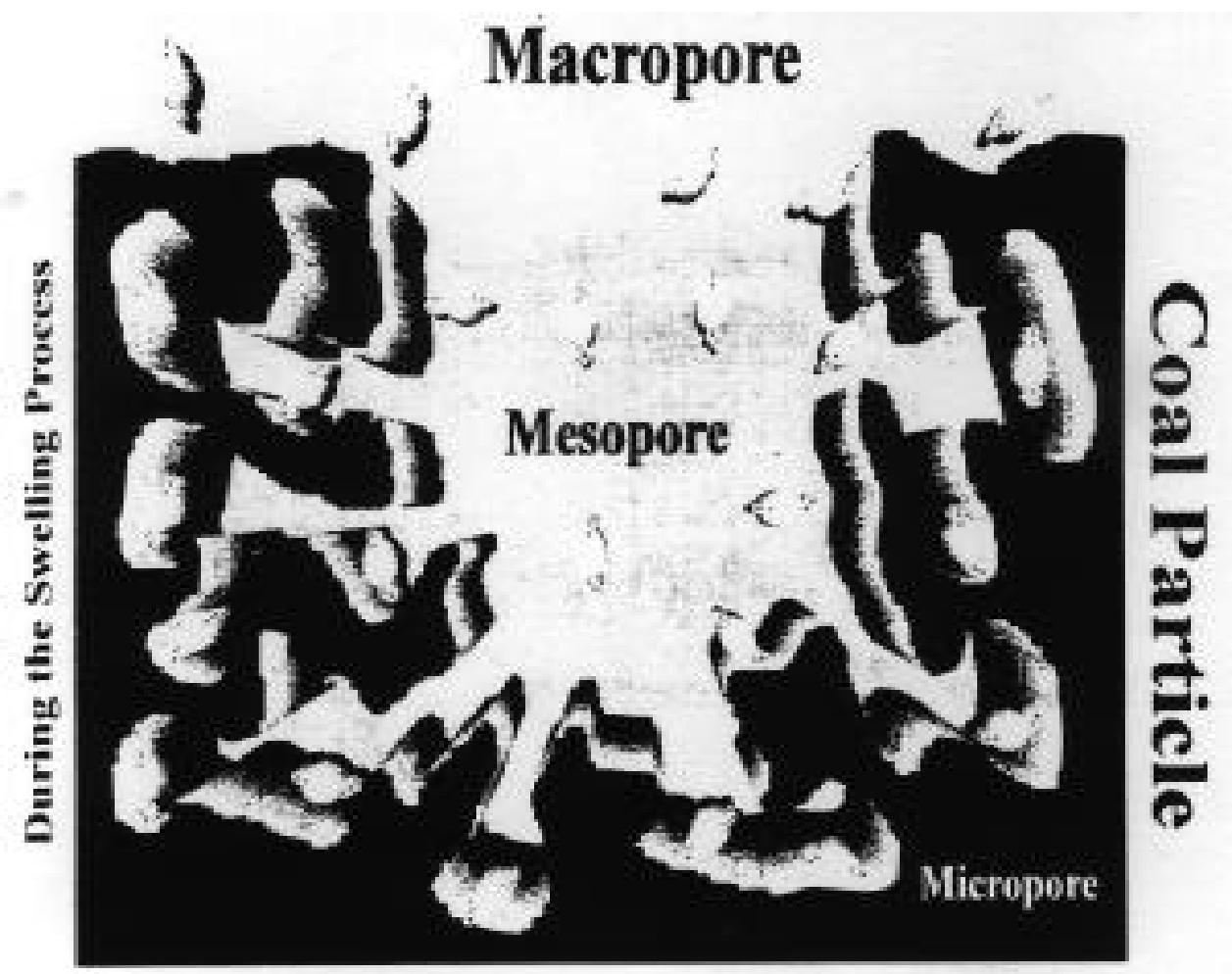

Figure 2. A schematic of the coal structure during swelling and the diffusion of the spin probes during this process.

After the spin probes have had a chance to diffuse into the coal structure, the swelling solvent is removed and the coal structure is allowed to re-collapse to its original state. At this point, some of the spin probes will be trapped in pores which are close to their own size, some will be in pores larger than their molecular size, and some will be simply lying on the surface of the coal in the mesopores or macropores as shown in Figure 3. 


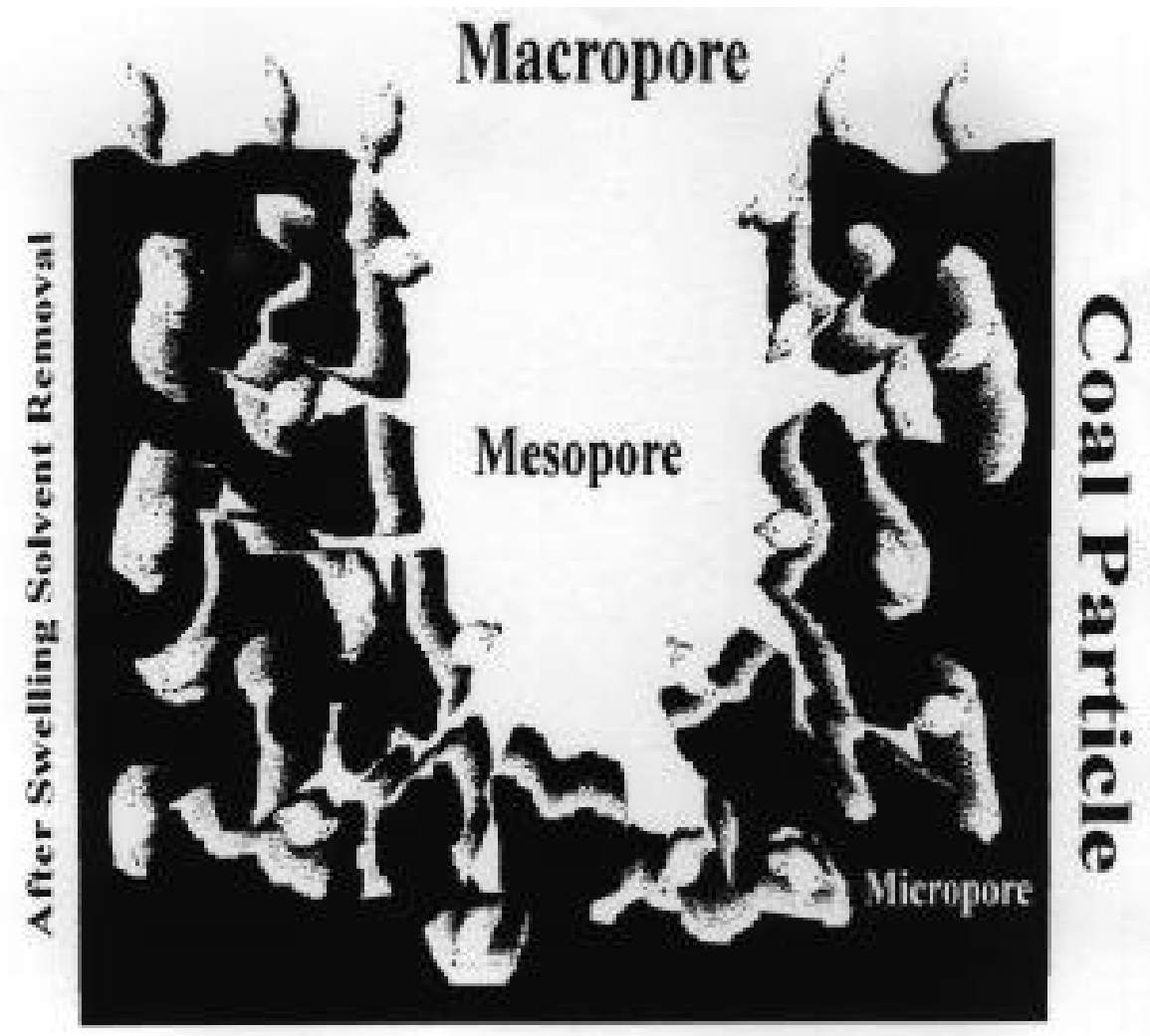

Figure 3. Illustration of the coal structure after the swelling solvent has been removed.

The next step is to remove the spin probes which are not trapped in pores or accessible regions which closely approximate their own volume. Since the spin probes are extremely soluble in cyclohexane which does not swell the coal in any way, the coal samples are washed with cyclohexane and vacuum dried to remove any of the solvent. This results in the intercalation of only the spin probes which are trapped in pores which restrict rotational or translational motion of the probe as shown in Figure 4. 


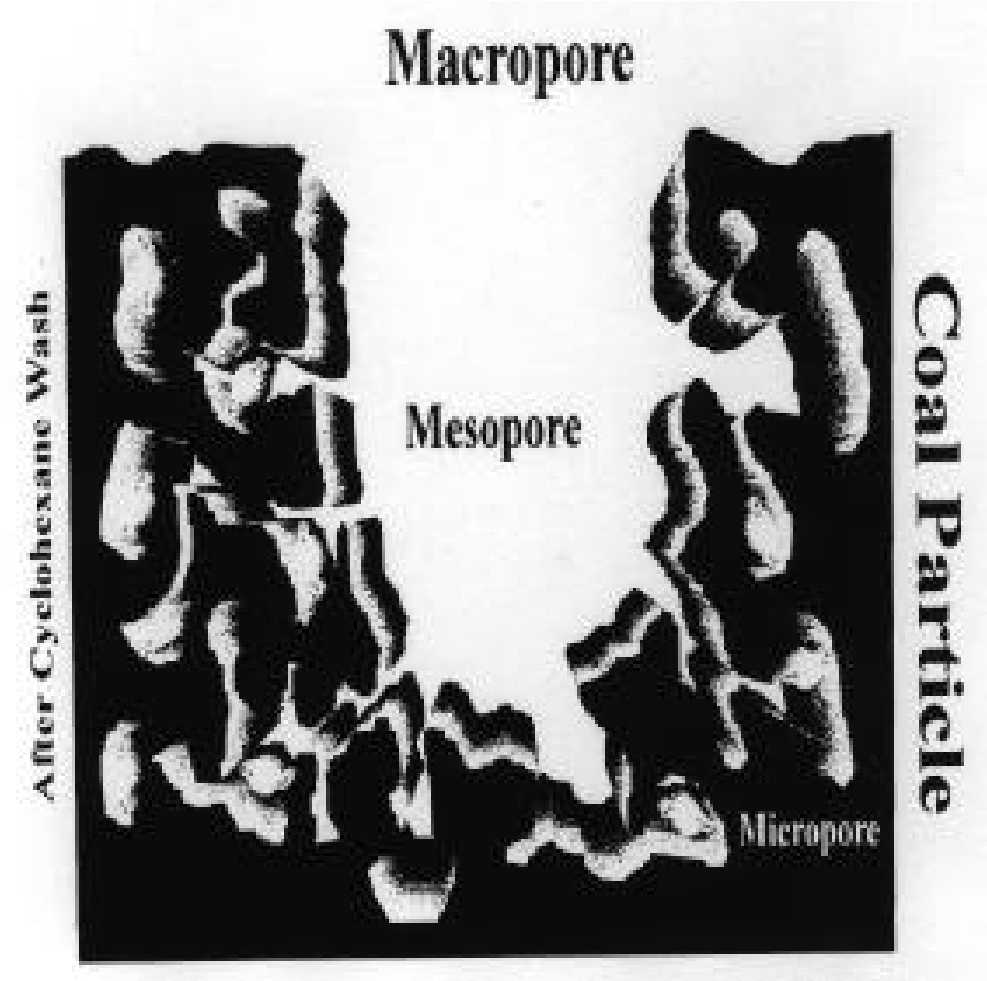

Figure 4. A diagram of the structure of the coal after the remaining spin probes have been washed off with cyclohexane.

During the swelling process, there are many types of interactions which are disrupted. Solvents which are strongly polar are capable of disrupting the hydrogen bonded cross-linking in coal. Additionally, the oxidation process during weathering may affect the covalently cross-linked character of the coal structure. In the following chapters it will become apparent that the EPR spin probe technique is very sensitive to changes in both the hydrogen bonded and covalently cross-linked structure in coal. In order to better explain some of the results obtained in the following studies, a cartoon is used to illustrate the proposed cross-link interactions in coal. This is shown in Figure 5.

In Figure 5, the line indicated by A represents a cross-link which isolates an individual micropore. Thus while the surrounding pores are accessible to the spin probes, in a solvent where this interaction is not disrupted, like toluene, the spin probes would not have access to this pore. However, if $A$ represented a hydrogen bond interaction, a more polar spin probe would disrupt $A$ and allow the spin probes access to this type of pore. The line indicated by $C$ represents a cross-link which blocks off a channel which connects some of the micropores. The spin probes would therefore only have access to the pores in front of this cross-link. The line shown by $B$ represents cross-linking between bedding planes in the coal structure. These cross-links limit access of the spin probes to the micropores to the channels connecting them. If they are disrupted, spin probes may have greater access to the micropore system, but may also lose the steric support provided by the bedding plane in trapping the probes. It may also be possible, given this view of the coal structure, that the bedding plane actually provides one side of the micropore, and its disruption could cause reformation of the micropore to a cup which could not effectively trap the spin probe, similar to removing one or more sides from a calixarene 


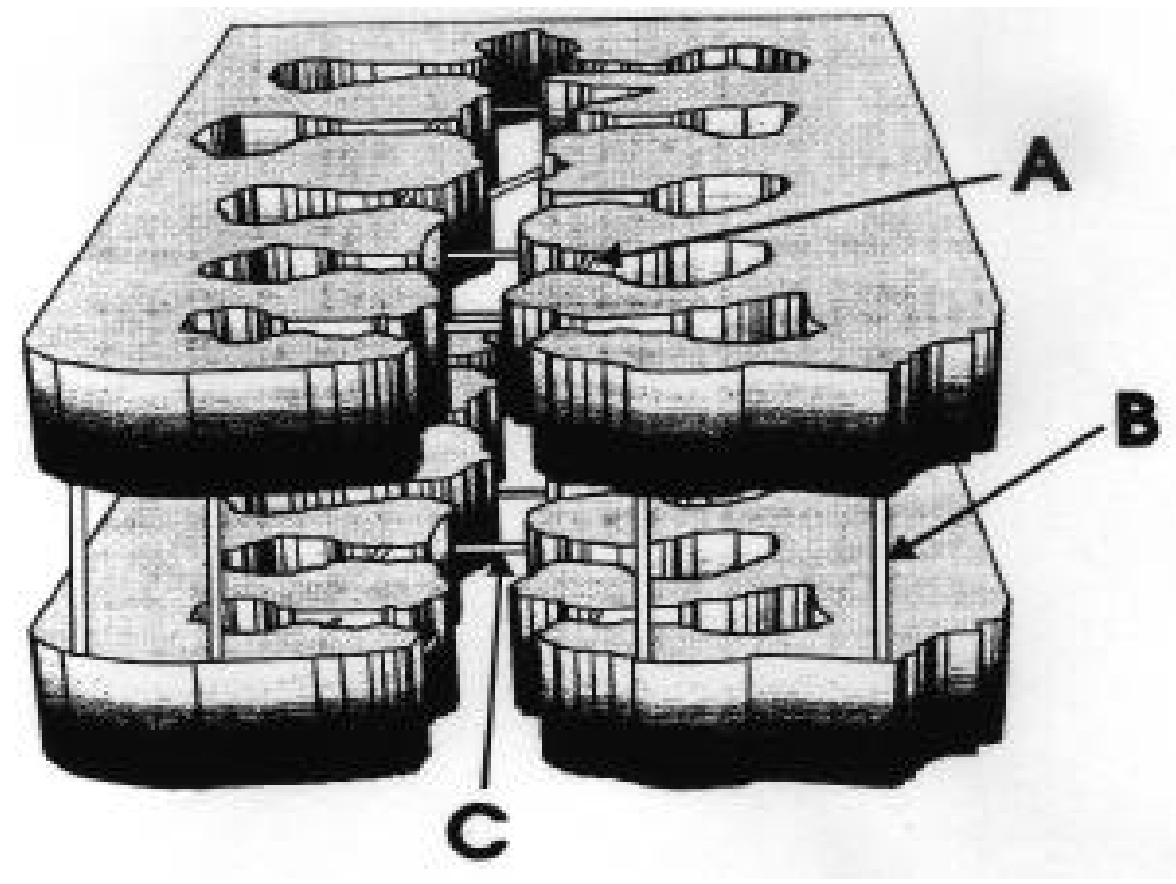

Figure 5. A cartoon of the cross-link structure in coal.

\section{Using Polymers to Model the Structure of Coal}

Although considerable evidence exists that coal is highly cross-linked, the nature and extent of covalent cross-links in coal is very uncertain. Cross-links can be of two types, covalent bridges and non-covalent interactions (i.e. charge-transfer, van der Waals, and hydrogen bond associations). Coal becomes more aromatic as coalification progresses (i.e., as rank increases). ${ }^{16}$ and becomes more closely packed up to the low volatile bituminous range ( $88 \%$ carbon, $\mathrm{dmmf}) .{ }^{17}$ This suggests that coal becomes more crosslinked as rank increases. However, the cross-links are not hydrogen bonds at high ranks, but rather van der Waals or covalent bonds. Nishioka and Larson, in a follow-up to the work of Larson and Mohammadi ${ }^{18}$ have shown that London dispersion forces increase in importance as rank increase ${ }^{19}$ due to an increase in the concentration of aromatic units. Covalent bridges are believed to consist predominately of ether linkage ${ }^{20}$ and methylene bridges. ${ }^{21,22}$ Ignasiak and Gawlak estimates that roughly half of all covalent cross-links might be ether linkages. ${ }^{23}$

Swelling studies have shown that coal behaves like a glassy polymer below its glass transition temperature $(\mathrm{Tg}) .^{24}$ Whether coal actually has a glass transition temperature is still open to question. ${ }^{25}$ Polymers have also been used to model solvent diffusion in coal. $^{26,27}$ These studies have been used to corroborate the assertion that coals behave like a glassy solid. More importantly, there is considerable evidence to show that coal is crosslinked well as by hydrogen bonding. If coal were entirely cross-linked by hydrogen bonding, coal in theory would be completely solubilized by repeated extraction with a 
strong base ${ }^{28,29}$ However, coal solubility and swellability decreases with rank. ${ }^{30}$ Further, phenolic oxygen is primarily responsible for hydrogen bonding in coals above the subbituminous rank, the hydroxyl content decreases with rank. This is because the oxygen substituents on the side rings have been converted to ether/furan (covalent) linkages. Since covalent bridges are not affected by solvents in any known way, ${ }^{24}$ it is not surprising that coal can only be partially solubilized. The polystyrene-divinylbenzene copolymers used in this study, however, do not exhibit any hydrogen bonding or similar polar interactions, thereby eliminating some of the more prominent interactions in coal. In addition to this, polymers have specific extents of covalent cross-linking. So, since these copolymers can be highly cross-linked with covalent interactions without the presence of hydrogen bonding, they are valuable models for studying the molecular accessibility as a function of covalently cross-linked character in coal.

Hydrogen bonded cross-linking plays an important role in coal structure. However, the contribution of covalent cross-linking in the coal structure is not well studied. Difficulties in studying the covalently cross-linked areas in coals arise from the interference caused by hydrogen bond interactions. Model polymers can be designed with no hydrogen bond interactions and well defined covalent cross-linking. Because of these limited similarities and significant differences between coal and polymers, considerable insight into molecular accessibility of coals during swelling can be gained by studying the swelling of polymers.

\section{The Weathering Process and Coal}

During weathering, coals are subject to both a loss of water and to oxidation. Both of these have been shown to have a strong effect on coal conversion. ${ }^{31,32}$ Lignites and subbituminous coals can contain over $30 \%$ water by weight, so drying is considered to be economically important in the conversion of low-rank coals. The transportation cost alone can be significant. However, exposure to air during the weathering process is an important consideration in all coal ranks. Changes in the physical and chemical structure of fresh Argonne Premium Coal Samples (APCS) during weathering have been previously followed using an EPR spin probe technique. ${ }^{4}$ This was possible by swelling the coal in a solution of spin probes containing a substituent capable of probing the chemical changes in coal. The substituents used in this study contained functional groups capable of being a hydrogen bond donor $(-\mathrm{COOH})$ or a hydrogen bond acceptor $\left(-\mathrm{NH}_{2}\right)$. It has been shown that the hydrogen bond donating ability increases in the series $-\mathrm{H}<<-\mathrm{OH}<-\mathrm{COOH}<-$ $\mathrm{NH}_{2}$. This feature is used to deduce the effect of oxidation on coal. To more clearly illustrate these polar interactions, a diagram of the hydrogen bond interactions exhibited between the coal structure and the polar spin probes is shown in Figure 6. In this figure, the hydrogen bond donor or acceptor character of each polar spin probe is shown interacting with the hydroxyl group (both a hydrogen bond donor and acceptor) of the coal structure. The thicker lines between the probe and the coal indicate stronger interactions and the thinner lines indicate weaker interactions. It is the goal of this research to use the EPR spin probe technique to investigate the contribution of oxidation on the swelling properties of coal during the weathering process. 


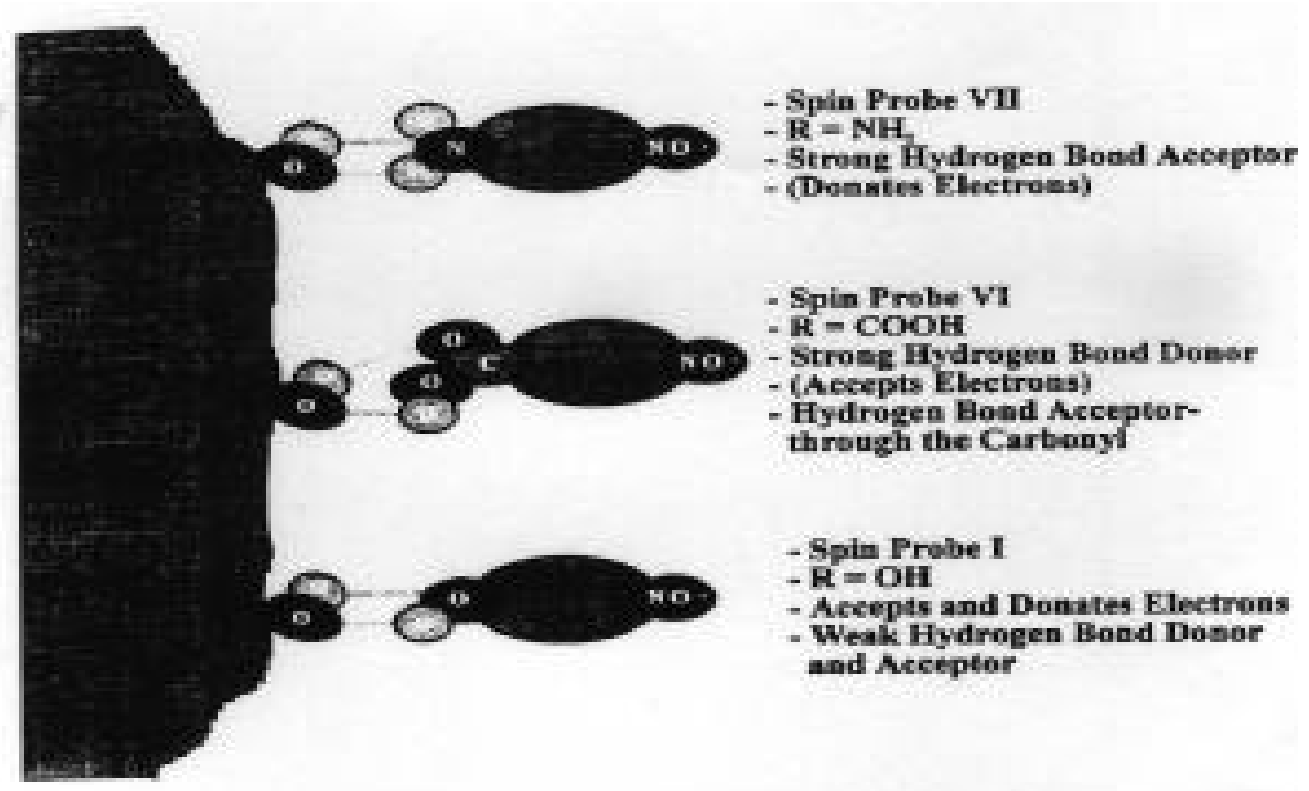

Figure 6. The hydrogen bond interactions of spin probes I, VI and VII with the hydroxyl groups in coal structure.

\section{Autoxidation}

To study the effect of oxidation during the weathering process, it is instructive to examine oxidation of other macromolecular systems. In this case, the oxidation of polymers has been extensively studied and can provide insight into the oxidation process in coal. The oxidative degradation of polymers proceeds by a method called hydroperoxide autoxidation $^{33}$ and is shown in the following steps.

Initiation:

$\mathbf{R}-\rightarrow \mathbf{R}^{\bullet}$

Propagation:

$\mathbf{R}^{\bullet}+\mathrm{O}_{2-->} \mathbf{R O O}^{\bullet}$

$\mathrm{ROO}^{\bullet}+\mathrm{HR}_{-}->\mathrm{ROOH}+\mathrm{R}^{\bullet}$

(3) (limited in coal by steric considerations)

Termination:

$\mathbf{R}^{\bullet}+\mathbf{R}^{\bullet}-\rightarrow \mathbf{R}-\mathbf{R}$ 
$\mathbf{R}^{\bullet}+\mathrm{ROO}^{\bullet}-\rightarrow$ R-O-O-R

$\mathrm{ROO}^{\circ}+\mathrm{ROO}^{-}-\mathrm{R}-\mathrm{O}-\mathrm{O}-\mathrm{R}+\mathrm{O}_{2}$

Functional Rearrangement:

R-O-O-R --> R-O-R+O

$\mathrm{ROOH} \rightarrow \mathrm{O}=\mathrm{R}-\mathrm{OH}$

HROORH $\rightarrow$ 2R-OH

R-O-O-R --> O-R-R=O

The new radicals formed in the propagation step (3) can then react with additional oxygen molecules. The hydroperoxide can also do further damage. If $\mathrm{R}^{\bullet}$ is an alkyl radical, the reaction proceeds relatively quickly. ${ }^{34}$ If $\mathrm{R}^{\bullet}$ is a quinone radical, it will not further react with oxygen to any significant extent. ${ }^{35}$ This step inhibits autoxidation. The reaction also takes place very slowly in aromatic systems or in any other system where the radical is delocalized. This is the case in coal.

It can be seen by examining these steps, that exposure of coal to oxygen could result in new radical locations in the coal structure. Also, the newly created peroxide radicals could bridge gaps across micropore cavities, allowing radical-radical interaction not previously available in the coal structure. In either case, the termination steps shown on the previous page (4), (5), and (6) are more likely to occur in coals during the oxidation process. All of these steps result in additional covalent cross-linking, and indeed this process has been used in forming polymeric substances as early as 1942.36 As indicated by previous studies, increased covalent cross-linking in coals would result in a decrease in molecular accessibility of spin probes to the coal structure. ${ }^{14}$

If termination of radicals does not occur, the formation of hydroperoxides prevails. Hydroperoxides are not very stable, and their decomposition results in the formation of alcohols (9), aldehydes (7), ketones (10) and acids (8) ${ }^{35}$ These reaction products are then subject to further oxidation and polymerization. The formation of these oxygenated functional groups in coal results in additional hydrogen bonding sites so that a larger amount of polar spin probes can be retained. This presents two processes which can be monitored by following the molecular accessibility of spin probes in coal.

An illustration of these processes is shown in the following figures. Coal contains enough free radicals initially so that the initiation step caused normally by incident radiation is not necessary for autoxidation to occur. An illustration of an average microchannel in the coal structure is shown in Figure 7 where radials are represented by $\mathrm{R}^{\circ}$. 


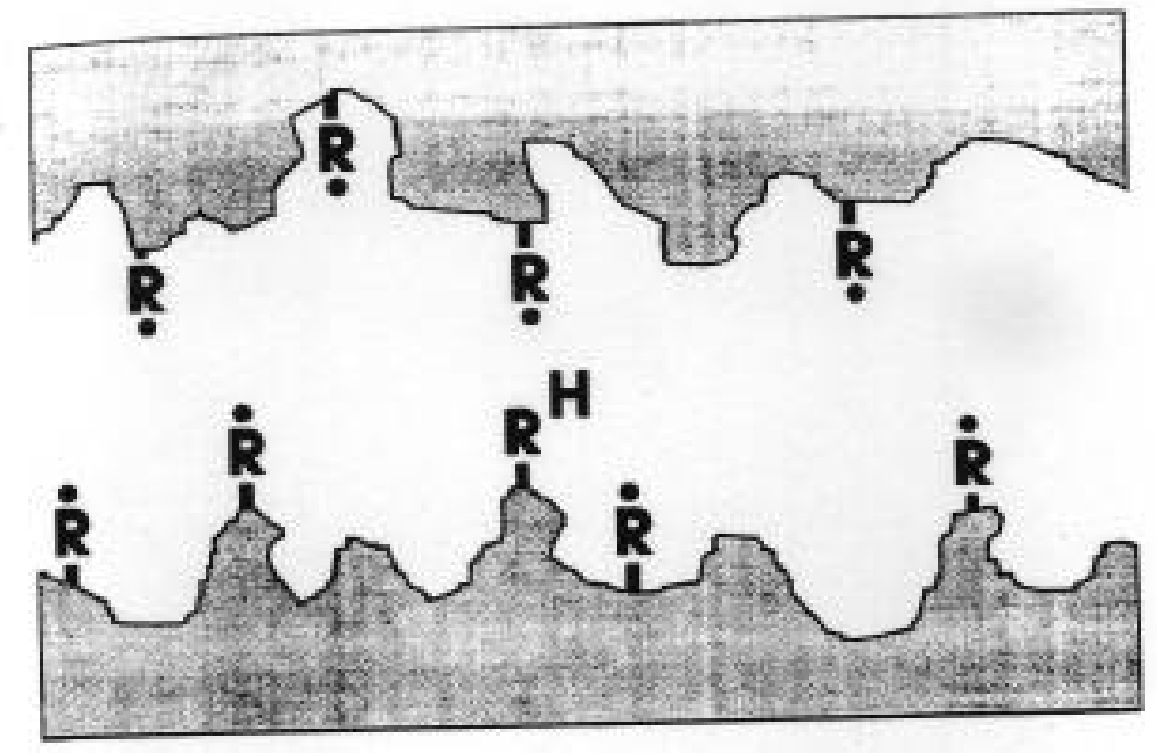

Figure 7. An illustration of a coal microchannel with radical sites.

Upon oxidation, the initial chemisorption of oxygen results in the formation of hydroperoxide radicals as shown in Figure 8.

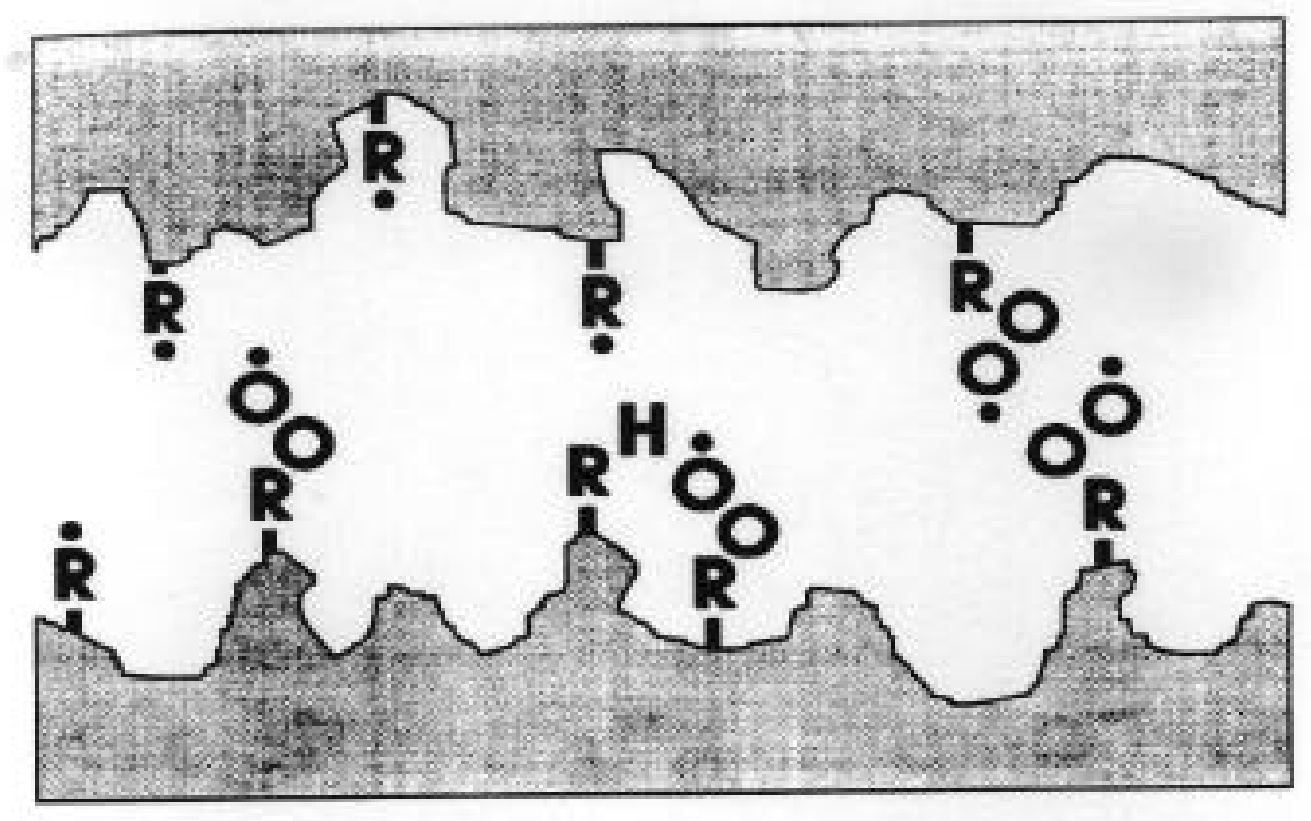

Figure 8. The formation of hydroperoxide radicals in the coal structure.

Once the hydroperoxide radicals have been formed, cross-linking can occur by the combination of the new hydoperoxide radical with existing radicals in the coal structure as shown in area $A$ in Figure 9. Likewise, two hydroperoxide radicals can combine with the release of oxygen to form a peroxide linkage as shown in area $C$ in Figure 9. The relocation of radicals is demonstrated by area $B$, where a hydrogen is removed from a 
neighboring group in the formation of a hydroperoxide. This relocated radical is now closer in proximity to other radicals in the coal structure.

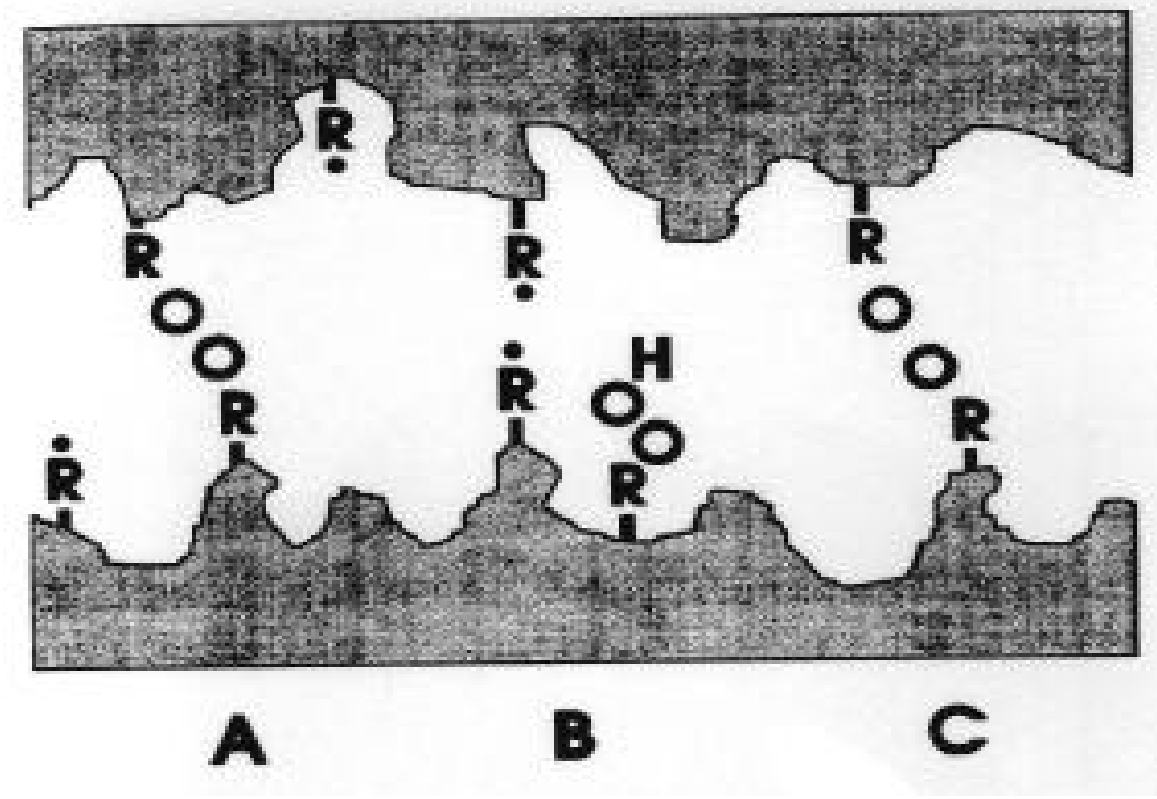

Figure 9. The formation of peroxide cross-links and the relocation of radical sites from hydroperoxide radicals.

The peroxide cross-links can then reform into ester linkages as shown by $A$ in Figure 10. Also, other covalent cross-links can be formed by the combination of relocated radical sites by other proximate radical sites as shown by $B$ in Figure 10. In the end, three different types of covalent cross-links have been formed. Further oxidation could cause additional covalent cross-linking and hydrogen bonded cross-linking by the increase in polar substituents in the coal structure. 


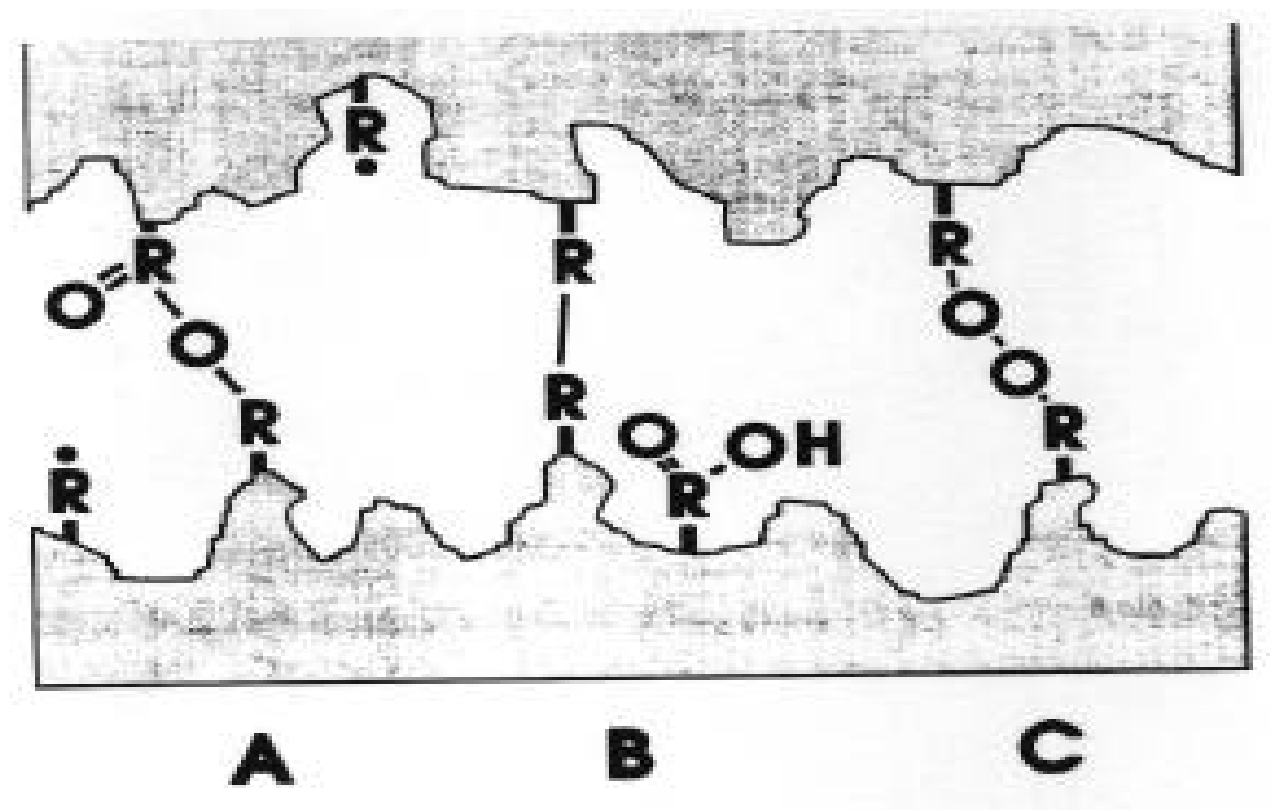

Figure 10. The formation of ether linkages in coal from peroxide cross-links and the formation of other covalent cross-links by combination of radical sites.

\section{Methods for Studying the Structure of Coal}

The porous structure of coal has been of concern for many decades. The use of catalysis in the conversion of coal is generally related to the surface area available to the catalyst. Since most of the surface area of coal lies in the micropore structure of the coal, the determination of this structure and its effect on catalyst accessibility are important in coal conversion. 37,38 The most popular and versatile method for determination of the porous structure of coal is the gas adsorption method. Although this method provides more information than $\mathrm{Hg}$ porosimetry or other physical methods, it does have some severe limitations. ${ }^{39}$ Several studies have given contradictory data, and it has been shown that micropore volumes can vary by more than an order of magnitude when different gases are used ${ }^{40}$ Adsorption techniques have yielded interesting results. Larsen has published a great deal of work in this area. ${ }^{40}$ The results of nitrogen adsorption showed surface areas of about $10 \mathrm{~m}^{2}$ at $77 \mathrm{~K}$ while carbon dioxide showed surface areas of about $215 \mathrm{~m}^{2} / \mathrm{g}$ at $195 \mathrm{~K}$ and cyclopropane $40 \mathrm{~m}^{2} / \mathrm{g}$ at $210 \mathrm{~K}$. Their assumption was that the carbon dioxide dissolved into the coal structure and was able to diffuse into pores which are not accessible to nitrogen because they are not connected by passages. The carbon dioxide molecules somehow diffuse through the solid structure of the coal. This could only be explained through the swelling of the coal upon carbon dioxide adsorption. The fact that cyclopropane shows a much higher surface area than nitrogen indicates that the structure must change as the temperature is decreased. If this is truly the case, how can gas adsorption be used to accurately determine the surface area of the micropore system in coals? It should be noted that the surface area determined by carbon dioxide is closer to the results obtained in X-ray diffraction experiments. However, these experiments and others use gas adsorption as evidence to support their own experimental data interpretations. This leads naturally to spectroscopic or imaging methods.

\section{SANS, SAXS and Light Scattering}

In the mid 1980's, methods using small angle neutron diffraction (SANS) and small angle x-ray diffraction (SAXS) were used with success to characterize the shape and 
size of the micropores in Illinois \#6 coal. ${ }^{41-46}$ It was determined from these studies that the micropores in low-rank coal had elongated cylindrical shapes which had diameters of about 5.2A. More recently, SAXS has been used to study the pore structure during fluid extraction. ${ }^{47}$ It was shown that micropore dilation was an irreversible process, whereas swelling of the macropore system was a reversible process. ${ }^{47}$ In 1981, Kalliat et al. used small angle x-ray scattering to show that there were three classes of pore systems in coal. ${ }^{41}$ These results were somewhat consistent with adsorption data interpretation at that time. Small angle x-ray scattering was used later on by Setek et al. in 1983 to determine the micropore structure of brown coal. 42 SAXS has also been used by Nemmers et al. in 1990 to study the porous structure of coal during fluid extraction. ${ }^{47}$ During various stages of fluid extraction of coal with THF, small angle x-ray scattering experiments showed that the extensive micropore structure formed during fluid exposure played a key role in the interaction between the fluid and the coal matrix needed for substantial extraction yields. ${ }^{47}$ It was determined by Tricker et al. in 1983 that small angle neutron scattering could be used to effectively determine pore size distributions in various ranks of coals. ${ }^{4}$ They felt that since the data obtained were substantiated by gas adsorption techniques, SANS could be used to probe coal porosity under circumstances where gas adsorption techniques were either severely limited or not possible. ${ }^{44}$ Likewise, in 1983, Kaiser and Gethner also used small angle neutron scattering to study pore distributions and pore accessibility in coal. ${ }^{45}$ It was determined that $\mathrm{D}_{2} \mathrm{O}$ was not able to penetrate uniformly through dried coals, and pore distributions were shown to be broad, extending over a $12 \mathrm{~A}$ to $500 \mathrm{~A}^{45}$ Later, in 1986 , Gethner used SANS to determine the void structure of Illinois \#6 coal. He determined that the microvoid volumes were elongated and possessed a well defined circular crossection of about 25A. ${ }^{3}$ Also, by comparison of aqueous and non-aqueous solutions, it was determined that the surface of the microvoid volume was primarily aliaphatic. ${ }^{43}$ In 1987 , Gethner used SANS and a combination of light scattering and absorption measurements to show that coal is a continuous distribution of voids rather than a well defined, discrete pore distribution, and to separate chemical changes from physical changes in coal during treatment and reactions.

\section{SEM and Imaging Techniques}

Another technique used recently involves SEM analysis during liquid metal infiltration to provide direct imaging of the micropore system.48 In 1991, Cody and Davis were able to directly image the pore space available to liquid metal (Wood's alloy) in a variety of coal ranks using SEM analysis. ${ }^{48}$ They determined that the pores in the lowest rank coal were roughly cylindrical in shape, but that they constituted a rough particle surface and not an interconnected cylindrical pore network. ${ }^{48}$ Pore space available to liquid metals in bituminous and higher rank coals appeared to be made up entirely of microcracks. ${ }^{48}$ Around this time, magnetic resonance imaging was also used to study pore structures during swelling. ${ }^{49}$ It was shown that coal swells anisotropically; that is, solvents enter the bedding planes and thus are not distributed uniformly in the coal structure. ${ }^{49}$

\section{NMR Spin Lattice Relaxation and Proton NMR}

Glaves et al. showed in 1988, that pore size distributions could be derived by deconvoluting measurements of the NMR relaxation spectra and applying a model to the pore fluid behavior. ${ }^{50}$ 


\section{Xe-129 NMR}

Recently, Xe-129 NMR studies have been used to determine average micropore volumes and diameters. ${ }^{51,52} \mathrm{Xe}-129 \mathrm{NMR}$ was used in 1990 by Wernet et al. to determine average micropore diameters in Illinois \#6 bituminous coal. ${ }^{51}$ After performing Xe-129 NMR experiments on spherocarb, which gave results very close to the manufacturer's specifications of microporosity, they found that the average micropore diameter of Illinois \#6 coal was 5.2 A. ${ }^{51}$ More recently, in 1991, Tsiao and Botto use this technique to determine the average micropore size of APCS coals and to investigate the effect of weathering on the average micropore size of coals. ${ }^{52}$ This information was then used to develop a model to approximate pore sizes and pore size swelling chacteristics. ${ }^{52}$

\section{EPR Spin Probe Method}

Finally, a technique using EPR to follow the inclusion of molecular probes in the micropore structure has been used to determine pore size and shape characteristics of a range of coals during the swelling process. ${ }^{3,5,6,11,53-55}$ This technique has also been used to follow changes in the micropore structure during weathering, oxidation, dehydration and short term oxidation. $4,7,8,15,56$ The EPR spin probe method was first used to study coal by Silbernagel et al. in 1981.11 Later, in 1985, Wuu and Kispert expanded this technique to determine micropore size distributions in coal during swelling. ${ }^{53}$ This EPR spin probe technique was further developed to study micropore size distributions and acid/base character of various ranked coals.6,7,54 More recently, changes in pore structure and wall chemistry of APCS coals upon swelling at different temperatures and with solvents of various polarities were determined by following the retention characteristics of the coals. ${ }^{3,5}$ The results showed that as the polarity of the solvent was increased, the shape of the micropores changed from spherical to cylindrical.3,5 Additional studies employing EPR spin probe techniques were performed on the breaking up of the hydrogen bonding between bedding planes. ${ }^{15}$ To gain insight into the accessible nature of the covalently cross-linked materials during swelling, the EPR spin probe method was used during the swelling of polystyrene-divinylbenzene copolymers which were cross-linked covalently to various extents ( $2 \%$ to $12 \%) .{ }^{9}$ The study showed that hydrogen bonding in coal has a much greater impact than percent cross-linking on the micropore structure and swelling character of coal. Despite this, significant amounts of guest molecules could be intercalated into the copolymer structure even though the copolymers were relatively non-porous. ${ }^{9}$ The effect of weathering on the structure and molecular accessibility of spin probes in coal was studied using the EPR spin probe technique. ${ }^{4}$ It was shown that after seven days of exposure to air, coals of most ranks exhibited significant changes in spin probe retention. 4 The lower ranked coals (Beulah Zap and Wyodak Anderson), were observed to undergo structural collapse which precluded retention of even the smallest probes, while medium ranked coals actually exhibited improved retention. ${ }^{4}$ A preliminary analysis of the data collected from the swelling of coals oxidized in a moisture free environment to differentiate between oxidation and the weathering process was published previously. ${ }^{7,8}$ Eight vacuum dried APCS coals were oxidized in a pure $\mathrm{O}_{2}$ environment and weathered in air, and the effects of oxidation alone on coal structure were determine by the intercalation of EPR spin probes. 7,8 It was shown that the removal of water was primarily responsible for the structural collapse observed in low-rank coals and for the increase in retention of polar spin probes in medium-rank coals. ${ }^{8}$ Coals oxidized in a pure oxygen environment showed an increase in retention by as much as a factor of five. ${ }^{7}$ Even the high-rank coals showed significant retention of polar spin probes after four days of exposure to oxygen. ${ }^{7}$ A study of $\mathrm{O}_{2}$ oxidation in all eight vacuum dried APCS coals is presented in the results sections. Studies of short term exposure of Illinois \#6 to dry argon and oxygen were initiated using 
the EPR spin probe technique. ${ }^{56}$ A detailed analysis of the obtained results has been reported.

\section{Spin Probes}

\section{EXPERIMENTAL}

The spin probe solutions were prepared in one millimolar concentrations for each of the spin probes VIII, VII, VI, I and XIII by adding 3.9mg TEMPO (spin probe VIII ${ }^{57}$ or 2,2,6,6-tetramethylpiperidine-1-oxyl); 4.2mg TEMPAMINE (spin probe VII or 4-amino2,2,6,6-tetramethylpiperidine-1-oxyl); 4.6mg of spin probe VI or 3-carboxy-2,2,5,5tetramethylpiperidine-1-oxyl; 4.3mg of TEMPOL (spin probe I or 4-hydroxy-2,2,6,6tetramethylpiperidine-1-oxyl); and finally $7.4 \mathrm{mg}$ of spin probe XIII ${ }^{57}$ or 4 -nonylamino2,2,6,6-tetramethylpiperidine-1-oxyl, to $25 \mathrm{ml}$ of toluene or pyridine. Fresh spin probe solutions were prepared immediately before use so that degradation of the radicals in the swelling solution would be minimized. The structure of these spin probes are shown in Figure 11. The spin probes are numbered consistent with other research done in this lab.<smiles>CC1(C)CC(O)CC(C)(C)N1[O-]</smiles>

I<smiles>CC1(C)CC(C(=O)O)C(C)(C)N1[O]</smiles>

VI<smiles>CC1(C)CC(N)CC(C)(C)N1[O-]</smiles>

VII<smiles>CC1(C)CC(O)CC(C)(C)N1[O-]</smiles>

VIII<smiles>CCCCCCCCCCCCCCCCCC</smiles>

XIII

Figure 11. Spin Probes I, VI, VII, VIII and XIII.

\section{Swelling the Coal Samples}

The APCS vials were opened in a glove bag under an argon environment, split into aliquots, and sealed in vials so that future experiments could be done on each particular coal sample. The samples were then removed and the aliquots stored for future use. The stored samples were placed under a minimum volume of dry argon so that a equilibrium could be quickly reached. Thirty milligrams of each coal sample was weighed out and placed in a glass vial. The coal was then covered with $2 \mathrm{~mL}$ of a 0.001 molar spin probe/swelling solvent solution and stirred on a magnetic stirrer for 18 hours to reach an equilibrium as shown in Figure 12 in steps 1, 2 and 3. After the coal was swelled for 18 hours, it was 
filtered using vacuum filtration and dried in a vacuum desiccator for three hours as shown in Figure 12 in steps 4 and 5. The sample was then stirred in cyclohexane, a non-swelling solvent, for three minutes to remove any spin probes on the surface or any spin probes not trapped in the coal structure, filtered again using vacuum filtration and dried in a vacuum desiccator for 1 hour as shown in Figure 12 in steps 6,7 and 8. Finally, 10mg of the coal was measured out into the EPR tube and put under a vacuum for 30 minutes. Each EPR tube was then heat sealed under the vacuum. A diagram of the swelling procedure is shown in Figure 12.

\section{Swelling Procedure}
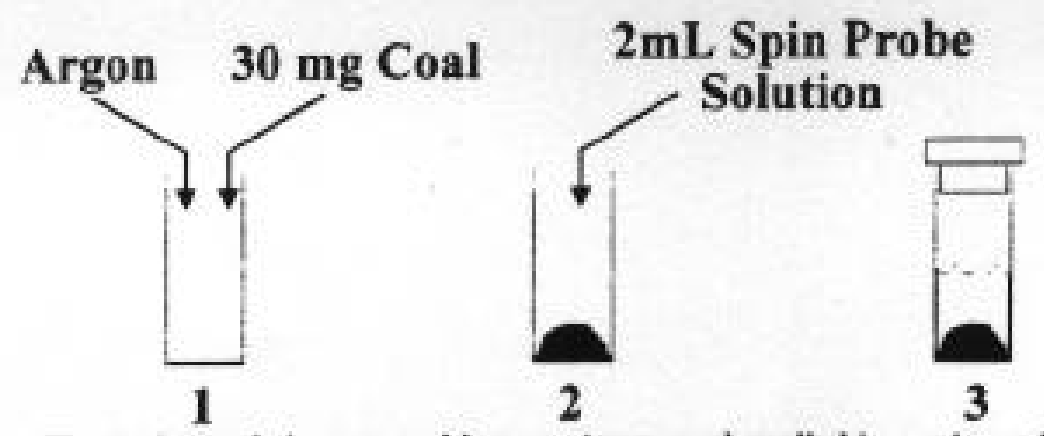

The coal sample is prepared in pure Argon and swelled in a spin probe solntion of swelling solvent for 18 bours

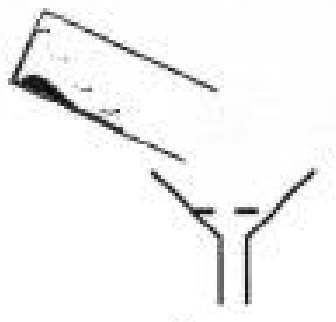

4

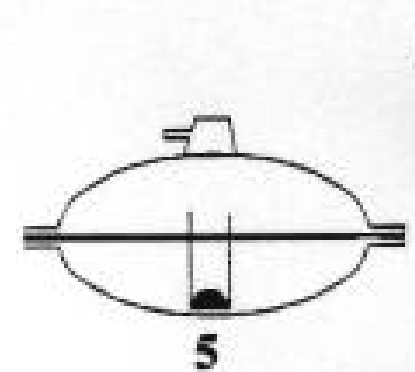

$3 \mathrm{~mL}$

Cyclohexane

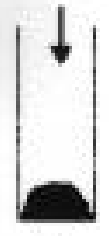

6

The swelled sample is then filtered, vacuum dried and washed with cycloherane
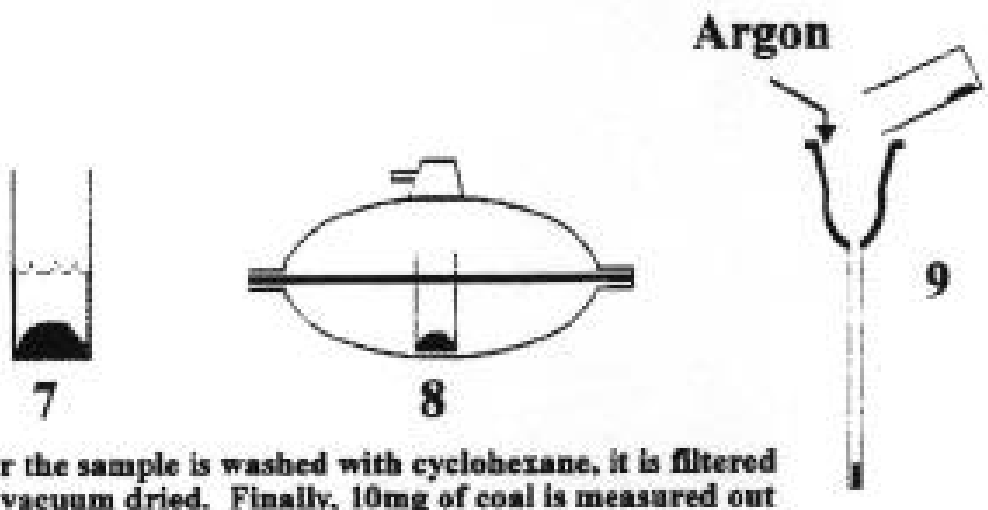

After the sample is washed with cycloberane, it is filtered and vacuum dried. Finally, $10 \mathrm{mg}$ of coal is measured out and sealed in an evacuated EPR tube.

Figure 12. The basic swelling procedure for the inclusion of spin probes in APCS coals swelled under ambient pressure and temperature. 


\section{Determining Spin Concentrations}

A powder spectrum of the nitroxide spin probes was obtained for each sample using a modified Varian E-12 spectrometer. Normal electronic double integration of the spectra could not be accomplished due to the interference of the coal radical signal in determining a baseline. Instead, the low field peak of the nitroxyl, which represents a proportional fraction of the total signal, was cut and weighed for compairison to the same portion of the powder pattern of a frozen nitroxyl. Spin concentrations were determined by calibration of the frozen spin probe spectrum to a $\mathrm{Cr}^{+++}$standard with a known number of spins. The measured spin probe concentration is representative of spin probes trapped in pores which closely approximate their own volume and limit movement since the isotropic signal of freely rotating spin probes is overwhelmed by the free radical spectra of the coal itself. The experimental operating conditions of the EPR spectrometer are shown below in Table 2.

Table 2. The experimental operating conditions of the EPR spectrometer.

Parameter $\quad \mathrm{Cr}^{+++}$Standard Coal Samples

$\begin{array}{lll}\text { Frequency } & 9.05 \mathrm{GHz} & 9.05 \mathrm{GHz} \\ \text { Magentic Field } & 5100 \mathrm{G} & 3210 \mathrm{G} \\ \text { Modulation } & 1 \mathrm{G} & 4 \mathrm{G} \\ \text { Power } & 0.5 \mathrm{~mW} & 3 \mathrm{~mW} \\ \text { Gain } & 6.3 \times 10^{3} & \text { Variable } \\ \text { Sweep Width } & 200 \mathrm{G} & 200 \mathrm{G} \text { or } 100 \mathrm{G} \\ \text { Scan Time } & 2 \mathrm{~min} . & 2 \mathrm{~min} . \\ \text { Time Constant } & 0.250 \mathrm{~s} & 0.250 \mathrm{~s}\end{array}$

The following equation was used to calculate the spin probe concentrations.

$$
S_{X}=\frac{S_{\text {std }} P_{X}^{0.5} A_{X} W_{X}^{2} G_{s t d} M_{\text {std }} g_{s t d}^{2}(S(S+1))_{\text {std }} * U_{x y}}{A_{\text {std }} P_{\text {std }}^{0.5} W_{\text {std }}^{2} G_{x} M_{x} g_{x}^{2}(S(S+1))_{x}}
$$

Where: $(\mathrm{x}=$ coal sample, and std $=$ chromium standard $)$

$\mathrm{S}=$ the number of spins

$\mathrm{A}=$ the area under the curve generated by the nitroxyl or standard

$\mathrm{W}=$ the scan width in gauss

$\mathrm{P}=$ the power in milliwatts

$\mathrm{G}=$ the gain setting

$\mathrm{M}=$ the modulation in gauss

$\mathrm{g}=$ the $\mathrm{g}$ value

$\mathrm{S}=$ the angular momentum quantum number

$\mathrm{U}=$ the transition probability for the chromium standard 


\section{Binary Swelling Solvent Systems}

The two spin probes used in this study were TEMPAMINE (VII) and TEMPO (VIII). The chemical structure of these spin probes are shown in Figure 11. Both have similar molecular volumes, but TEMPAMINE contains an amino functional group which exhibits polar interactions with available hydrogen bonding sites in the coal structure. $200 \mathrm{~mL}$ of each swelling solvent/spin probe solution were prepared in 1 millimolar concentrations of TEMPAMINE and TEMPO in toluene. Each spin probe solution was split into 16 aliquots of $10 \mathrm{~mL}$ and spiked with pyridine in the amounts of $0.000 \mathrm{~mL}$, $0.001 \mathrm{~mL}, 0.002 \mathrm{~mL}, 0.005 \mathrm{~mL}, 0.007 \mathrm{~mL}, 0.010 \mathrm{~mL}, 0.020 \mathrm{~mL}, 0.040 \mathrm{~mL}, 0.060 \mathrm{~mL}$, $0.080 \mathrm{~mL}, 0.100 \mathrm{~mL}, 0.200 \mathrm{~mL}, 0.300 \mathrm{~mL}, 0.400 \mathrm{~mL}, 0.500 \mathrm{~mL}$ and $0.600 \mathrm{~mL}$ for BeulahZap and Wyodak-Anderson coals, and $0.000 \mathrm{~mL}, 0.005 \mathrm{~mL}, 0.010 \mathrm{~mL}, 0.020 \mathrm{~mL}$, $0.040 \mathrm{~mL}, 0.060 \mathrm{~mL}, 0.080 \mathrm{~mL}, 0.100 \mathrm{~mL}, 0.200 \mathrm{~mL}, 0.300 \mathrm{~mL}, 0.400 \mathrm{~mL}, 0.500 \mathrm{~mL}$, $0.600 \mathrm{~mL}, 0.700 \mathrm{~mL}, 0.800 \mathrm{~mL}$ and $0.900 \mathrm{~mL}$ for Illinois \#6 coal. A vial containing each APCS coal (Beulah-Zap, Wyodak-Anderson and Illinois \#6) was opened under an argon environment and aliquots immediately distributed into vials, which were then covered with $2 \mathrm{~mL}$ of a toluene spin probe solution spiked with pyridine. Each sample was then swelled for 18 hours in each of these binary solutions. The samples were then prepared as previously described and the concentration of the spin probes were determined by EPR spectroscopy.

\section{Weathering}

Samples of eight APCS coals (Beulah Zap, Wyodak Anderson, Blind Canyon, Illinois \#6, Lewiston-Stockton, Pittsburgh \#8, Upper Freeport and Poccahontas \#3, defined previously) ${ }^{2}$ were stored in vials which were exposed to the air but protected from dust or other contaminants during the period of exposure. During the weathering period, the samples were agitated each day to insure complete exposure of the coal to the air. At various points during the weathering period of six months ( 0 days, 7 days, 14 days, 35 days, 64 days, 114 days and 180 days), samples were swelled in one millimolar solutions of each spin probe in the swelling solvents toluene and pyridine. The spin probes used for this study have been shown previously in Figure 11, VI (3-carboxy-2,2,5,5tetramethylpyrrolidine-1-oxyl), VII (TEMPAMINE) and VIII (TEMPO). Thirty mg of each coal was mixed with $2 \mathrm{~mL}$ of the pyridine or toluene spin probe solution. The mixture was then stirred for 18 hours so that the swelling would reach an equilibrium. The coal was then filtered and vacuum dried for 2 hours. The samples were then washed with cyclohexane to remove any spin probes not trapped in the coal structure. The samples were again vacuum dried and then sealed in the evacuated EPR tubes. The concentration of spins in each sample was calculated from the EPR spectra as previously described.

\section{Vacuum Drying}

The coals used in the weathering experiments on vacuum dried coal samples and the $\mathrm{O}_{2}$ oxidation experiments were vacuum dried in a vacuum desiccator at $10^{-4}$ to $10^{-5}$ torr for 18 hours.

\section{Oxidation in Pure Oxygen}

Samples of eight vacuum dried APCS coals (Beulah Zap, Wyodak Anderson, Blind Canyon, Illinois \#6, Lewiston-Stockton, Pittsburgh \#8, Upper Freeport and Poccahontas \#3, defined previously2) were stored in vials under pure oxygen for various periods of time in a glove bag. During the period of oxidation, the samples were agitated each day to insure complete exposure of the coal to the oxygen. At various points during the oxidation 
period ( 0 days, 4 days, 8 days, 15 days and 36 days and 64 days), samples were swelled in one millimolar solutions of each spin probe in the swelling solvents toluene and pyridine. The spin probes used for this study are shown previously in Figure 11, VI (3carboxy-2,2,5,5-tetramethylpyrrolidine-1-oxyl), VII (TEMPAMINE) and VIII (TEMPO). Thirty milligrams of each coal was mixed with $2 \mathrm{~mL}$ of the pyridine or toluene spin probe solution and swelled as described previously and shown in Figure 12. The concentration of spins in each sample was calculated from the EPR spectra obtained from the sample.

\section{Short Term Oxidation and Dehydration}

The vial containing the APCS coal Illinois \#6 was opened in a moisture free, pure argon environment and aliquots of coal immediately placed into an apparatus constructed to allow the flow of only pure argon or oxygen over the surface of the fresh coal. A sample of fresh coal was also swelled in each of the spin probe solutions to establish a point of no oxidation or dehydration. It should be noted that the coal was exposed to the argon environment for 10 to 15 seconds before it was placed into the swelling solvent. One aliquot of the coal was exposed to argon and the other to oxygen. A flow of approximately $40 \mathrm{~mL}$ per minute of each gas was maintained through the coal sample for 30 seconds, 5 minutes and 50 minutes. The coal was then swelled in a spin probe solution of toluene or pyridine for 18 hours. The swelling solvent was removed and the spin probe retention was measured by EPR. Samples were replicated so that some indication of reproducibility could be obtained. An illustration of this process is shown in Figure 13.

The three spin probes used in this study are show in Figure 11. Spin probes 3carboxy-2,2,5,5-tetramethylpiperidine-1-oxyl (VI), TEMPAMINE (VII) and TEMPO (VIII) were chosen so that changes in both physical and chemical structure could be observed. All three have similar molecular volumes but different chemical reactivites with the coal structure.

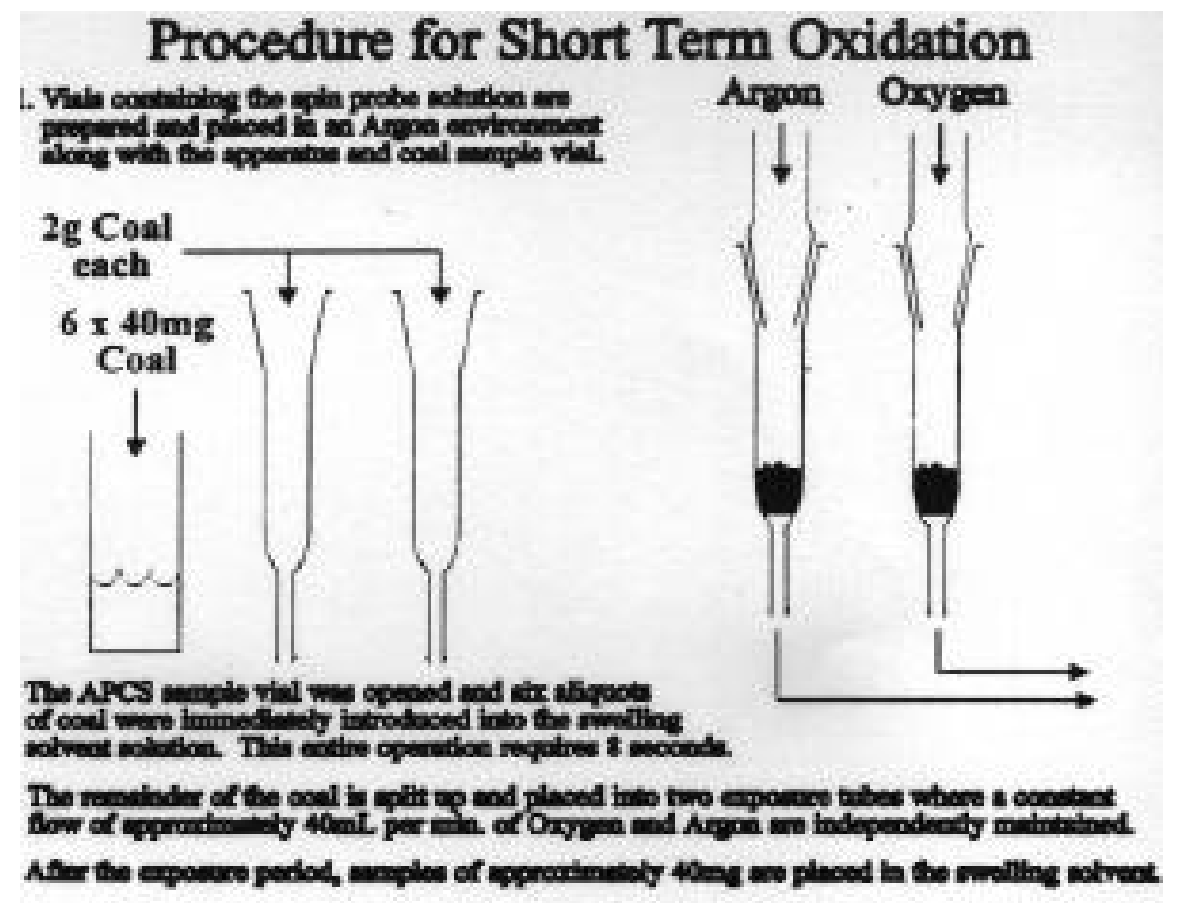

Figure 13. The procedure for preparing the Illinois \#6 coal for short term oxidation and dehydration. 


\section{High Presssure Swelling}

The spin probes used for this study, I (TEMPOL), VII (TEMPAMINE) and VIII (TEMPO), have been shown previously in Figure 11. The spin probe solutions were prepared as previously described, and $30 \mathrm{mg}$ of the APCS coal Illinois \#6 were added to a sample vial made for the high pressure vessels. Two milliliters of spin probe solution were added to the vial. The pressure vessels were prepared by flushing with argon, and the sample vial containing the coal and the spin probe solution was quickly inserted into the vial as shown in Figure 14. The pressure vessels were sealed and charged with argon at 0psig, 200psig, 400psig and 800psig. Because magnetic stir bars could not be used in the pressure vessels, the vessels were put into a shaker for 18 hours to agitate the samples during swelling. The sample was then prepared as previously described and shown in Figure 12 by steps 6 through 9.

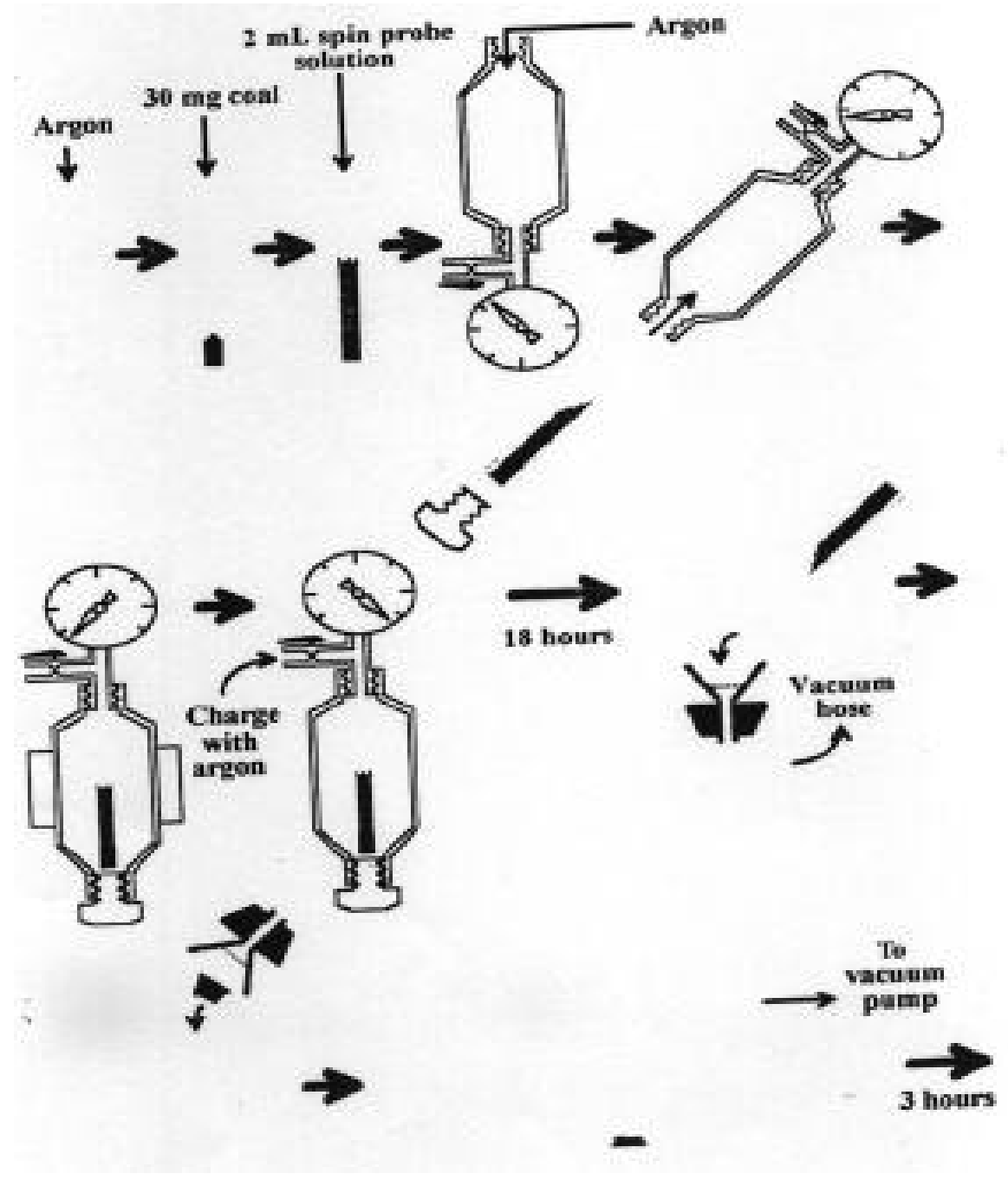

Figure 14. The procedure used for high pressure swelling studies. 


\section{Surface area Determination and Errors}

The surface areas of the polymers were determined by N2 BET surface analysis using a Leeds \& Northrup Instruments Surface Area Analyzer Model 4200. It was determined that 150 beads weighed 0.00056 grams. So with each bead having a radius of $3.21 \times 10^{-5} \mathrm{~m}$, the surface area for a non-porous bead would be $1.2 \times 10^{-8} \mathrm{~m}^{2} / \mathrm{sphere}$ or $0.003 \mathrm{~m}^{2} /$ gram. This is much lower than the experimentally determined surface area of about $0.1 \mathrm{~m}^{2} / \mathrm{gram}$. It can be easily seen that these polymers are porous, but have porosities which are at least one order of magnitude lower than coal which has N2 BET surface area of 1 to $10 \mathrm{~m}^{2} / \mathrm{gram}$. A swelling experiment also revealed that pyridine was a much better swelling solvent than toluene; the volume increase was two-fold greater after 18 hours of swelling.

In many of the experiments performed, three to six independent samples were prepared for each set of experimental conditions. The experimental procedure previously described has been refined from previous studies using the EPR spin probe method so that the experimental error could be minimized. After a complete study of each part of the experimental procedure by Dr. Wojcheck Sady, it was determined that most of the experimental error was due to inconsistent handling of the coal before and after swelling. Subsequent studies showed that much of the experimental error could be eliminated if only $10 \mathrm{mg}$ of the impregnated coal sample is measured out into the EPR tube before the final evacuation and sealing.

The absolute error in the measurements of the spinprobe concentrations made using the EPR spin probe method has been determined to be in the range of $25 \%$ to $50 \%$. However, as will be seen in subsequent chapters, the accuracy of the absolute spin concentration is of little concern to these studies. The relative measurements in spin concentration instead provide indication of changes in the coal structure as experimental conditions are varied.

The maximum error in the precision of the measurements in all of the studies except for short term oxidation is shown for the observed range of spin probe concentrations in Table 3.

Table 3. The maximum error in the precision of spin probe concentration measurements.

\section{Spin Probe Concentration}

$$
\begin{aligned}
& 10^{10} \text { spins per gram } \\
& 10^{18} \mathrm{spins} \text { per gram } \\
& 10^{17} \text { spins per gram } \\
& 10^{16} \text { spins per gram } \\
& 10^{15} \text { spins per gram }
\end{aligned}
$$

Error (max.)

$$
\begin{array}{r}
3 \% \\
5 \% \\
10 \% \\
20 \% \\
100 \%
\end{array}
$$

In very few cases did the error in precision exceed these values, and in these cases the data was not used.

In looking at spin probe retention data, it can be seen that the retention character of the coal follows a general decrease from the lower ranked coals to the high-rank coals. There are two notable exceptions in this trend. Illinois \#6 and Blind Canyon coals, although similar in chemical composition, exhibit completely different behavior in the inclusion of EPR spin probes. The same difference is observed between LewistonStockton and Pittsburgh \#8 coals. Much of these differences may be accounted for by the difference in ash content of these coals as previously discussed. In any case, the 
differences in the retention character of these two coal pairs can cause difficulty in the interpretation of EPR spin concentrations as a function of rank.

Three dimensional were used to show the effect of weathering as a function of rank. These plots are used to show strong changes by major features.

The largest experimental errors were seen in the short term oxidation studies. In this case, the error bars are representative of the range of the entire spectrum of the data obtained for each experimental condition. Where error bars are not shown, the range of data points did not vary by more than an amount represented by the width of the symbol. The short term oxidation experiments are very difficult to run, and require a lot of preparation. Because of this limitation and the need to do replicate samples, only four experimental conditions could be studied for each spin probe/solvent combination. Since the sensitivity of the spin probe method to the effects of short term oxidation or dehydration was unknown, the exposure times chosen were varied by an order of magnitude each time ( 0 minutes, 0.5 minutes, 5.0 minutes and 50 minutes). Measurements for the retention of the spin probes in fresh coal were repeated because the experimental method used in short term oxidation studies is so different from previous ones.

\section{RESULTS}

\section{VACUUM DRIED COALS OXIDIZED BY O2}

\section{Introduction}

It is possible to determine which types of oxygenated products are being formed during the oxidation process because their hydrogen bonding characters are greatly varied. If hydroxyl groups are being formed, we would expect to see an increase in both of the polar spin probes since the hydroxyl group acts both as a hydrogen bond donor and acceptor. If ketones or quinones are being formed, a strong increase in the retention of spin probe VI (the carboxyl spin probe) would be expected, while the retention of spin probe VII (the amino spin probe) would be affected minimally. If carboxyl groups are being formed, an increase in the retention of spin probe VII would be expected with minimal changes in the retention of spin probe VI.

Looking at the overall picture of the oxidation process in coal as discussed previously, it can be seen that initially a decrease in retention would be expected because of the increased cross-linking and lack of structural accessibility of the spin probes. This decrease would be observed in coals swelled in both toluene and pyridine since neither solvent is known to disrupt covalent cross-linking in coal. As the concentration of free radicals in the coal is decreased, this process becomes less significant, and the production of hydroperoxides becomes more important. As the oxidation process is continued, production of hydroxides causes both polar spin probes to be more strongly retained. As the hydroxyls are oxidized further to ketones, the retention of spin probe VI becomes stronger than spin probe VII. However, as the ketones and hydroxyls are further oxidized to carboxylic groups, this trend reverses and spin probe VII is retained more strongly than spin probe VI. Of course, since the reaction products can form cross-links with other sections of the coal, the over-all process can be extremely convoluted.

From the following scheme ${ }^{58}$ it can be seen that the continued exposure to oxygen should reach an equilibrium in which a large percentage of the oxygenated coal is in the carboxylic acid form. 
Autoxidation of

primary $\mathrm{OH}$ to aldehydes

primary $\mathrm{OH}$ to esters

primary $\mathrm{OH}$ to carboxylic acid

secondary $\mathrm{OH}$ to ketones

secondary $\mathrm{OH}$ to carboxylic acid

ketones to carboxylic acids

phenols to benzoquinones

Certainly, as the production of hydroperoxides reaches an equilibrium state, further oxidation would promote the production of carboxylic acids. At long periods of exposure to oxygen, an increase in the retention of spin probe VII should be seen.

The use of toluene as a solvent allows us to look at the hydrogen bonded structure of the coal since it does not disrupt these interactions to any significant extent. The use of pyridine, on the other hand, allows an examination of changes in the covalently crosslinked structure since it is known to disrupt all of the hydrogen bond interactions in the coal structure without affecting the covalent cross-links.

\section{Oxidation of Coal as a Function of Rank}

\section{Spin Probe VI}

The data is plotted three dimensionally for ease of analysis since so many samples were collected. The spin probe retention is expressed in terms of concentration in spins per gram versus the oxidation period in days and \% carbon (dmmf) for each spin probe/swelling solvent combination. The coals were vacuum dried and put in a moisture free oxygen environment so that only the effects of oxidation on the coals could be studied. Only the results of coals swelled in toluene are discussed in this section. The structural changes observed there for include changes to the hydrogen bonded structure of the coal. These results will be discussed in detail with the results of pyridine swelled coals for each individual coal later on in this chapter.

Figure 15 represents the effects of oxidation on vacuum dried coals swelled in toluene with spin probe VI. The front edge parallel to the Days of Oxidation axis shows the transition that Beulah-Zap goes through during the oxidation process with respect to the polar spin probe VI. It is shown that Beulah-Zap undergoes by a structural collapse upon vacuum drying. Little change in retention is observed during the first eight days of oxidation. However, after eight days, a continued increase in retention of spin probe is observed until after 64 days a retention of $7.2 \times 10^{18}$ spins per gram is observed. The structure must open during the oxidation process such that large amounts of spin probe VI are retained, because toluene is know to have little effect on breaking cross-links or other intramolecular interactions. ${ }^{3}$

Wyodak-Anderson, like Beulah-Zap, starts out with very little retention of spin probe VI and is not significantly affected by oxidation until 8 days of exposure to oxygen. At 8 days, the spin probe concentration increases to about $4 \times 10^{18}$ spins per gram (shown in Figure 15 at point $f$ ). However, after the increase at 8 days, there is little change until 64 days of exposure where at point $a$, a sudden relative decrease in spin probe retention is observed. This would indicate a structural change where the spin probe was no longer able to access the coal structure, or a change in chemical structure which would limit the polar interactions between the coal structure and the spin probe. 


\section{Spin Probe V Retention in $\mathrm{O}_{2}$ Oxidized Coal Swelled in Toluene}

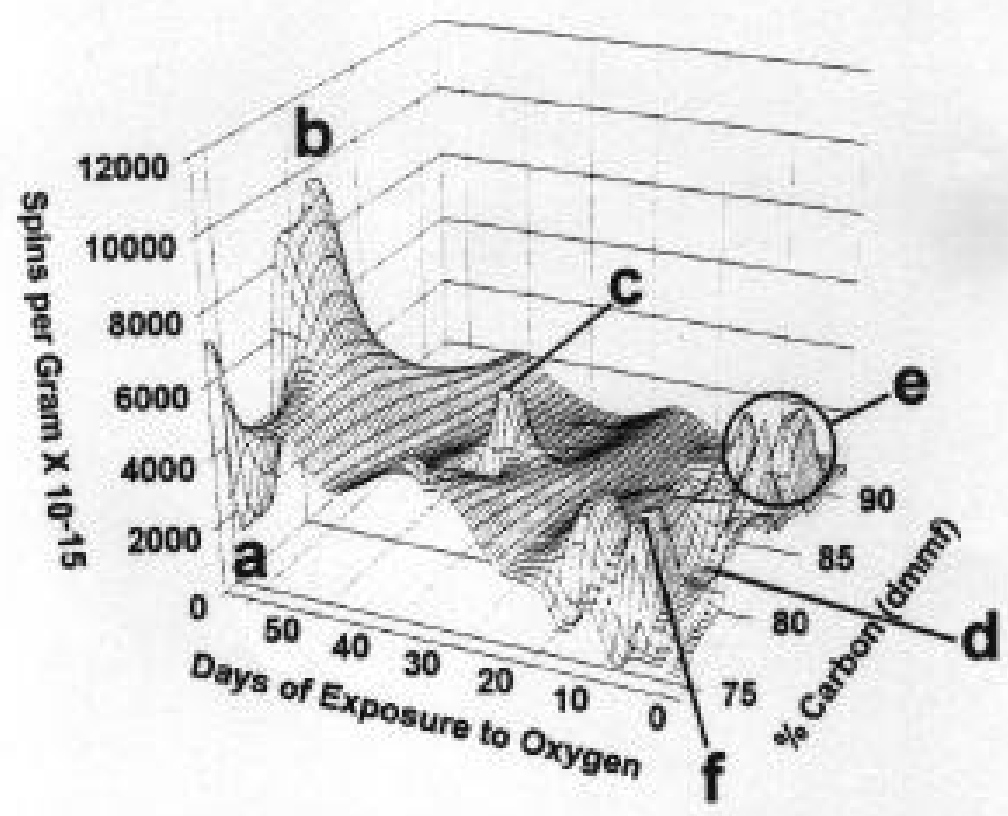

Figure 15. The retention of spin probe VI in $\mathrm{O}_{2}$ oxidized coals swelled in toluene, expressed as a function of both rank (percent carbon) and exposure period (days of exposure to oxygen) up to an exposure period of 64 days.

Blind Canyon exhibits a decrease of spin probe retention after 8 days of exposure to oxygen (point, followed by a steady increase in retention of spin probe VI until at 64 days of exposure to oxygen (point $b$ ), it retains nearly $1 \times 10^{19}$ spins per gram, which is five times the amount retained in Blind Canyon coal weathered for 64 days, indicating a large increase in oxidized material.

The high-rank coals, (Pittsburgh \#8, Lewiston-Stockton, Upper Freeport and Poccahontas \#3) which showed little change upon vacuum drying for coals swelled in toluene for spin probe VI, showed significant changes in retention characteristics after just four to eight days oxidation in oxygen (see area $e$ in Figure 15 or the expansion of Figure 15 given in Figure 16). For example, after 8 days of exposure to oxygen, Upper Freeport shows a retention of $3.7 \times 10^{18}$ spins per gram. Initially, the spin probe retention was 1.1 $\mathrm{x} 10^{17}$ spins per gram while the highest retention observed for Upper Freeport coal weathered in air for 8 days was $4.5 \times 10^{17}$ spins per gram. Both of these results are around an order of magnitude less than the results obtained for Upper Freeport oxidized in oxygen.

The effect of weathering vacuum dried coals in air and the effect of weathering fresh coals in air are presented in Figures 17 and 18 respectively for measurements of the retention of spin probe VI for 36 days. It is apparent that oxidation has a larger effect on molecular accessibility than vacuum drying during the weathering process. However, the presence of water greatly affects oxidation of coals and alters the retention characteristics of most coals. It is noteworthy to compare the results of the oxidation of vacuum dried APCS coals in $\mathrm{O}_{2}$ given in Figure 15 for 64 days with a similarly plotted curve (Figure 19) for spin probe VI for fresh APCS coals weathered in air. By comparing Figures 15 and 19, it 
can be seen that oxidation of coals for up to 64 days can result in a large increase in accessibility for polar spin probes into the coals structure, even for high-rank coals.

\section{Spin Probe VI Fetentlon in $\mathrm{O}_{2}$ Oxidized Coal Smolled in Toluene}

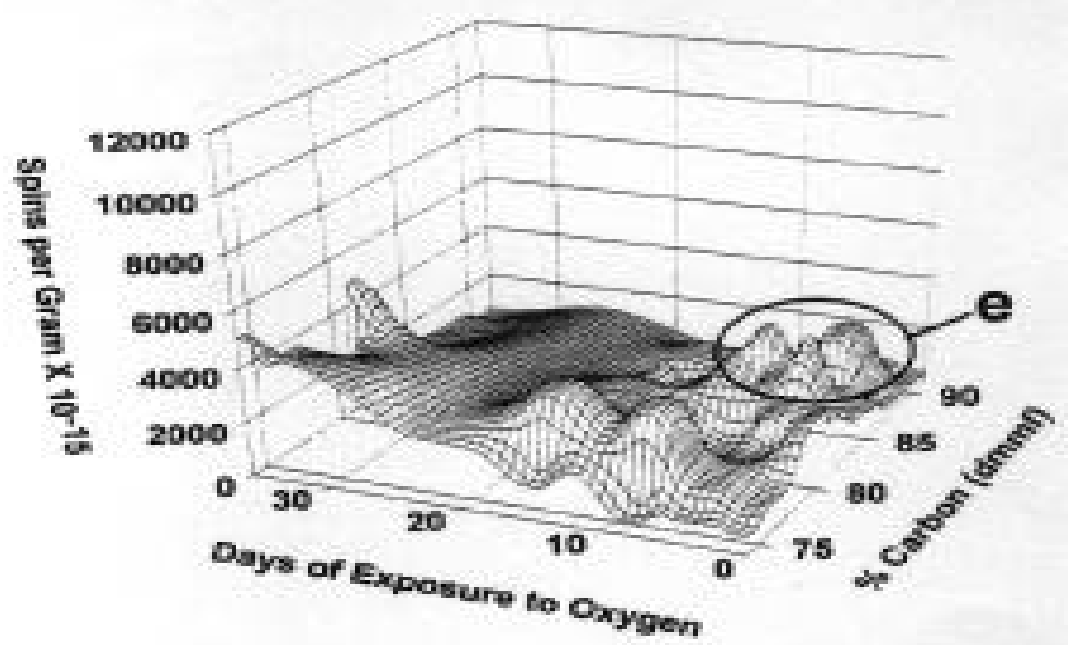

Figure 16. The retention of spin probe VI in $\mathrm{O}_{2}$ oxidized coals swelled in toluene, expressed as a function of both rank (percent carbon) and exposure period (days of exposure to oxygen) for up to 36 days.

\section{Spin Probe VI Retention in Weathered, Vacuum Dried Coals}

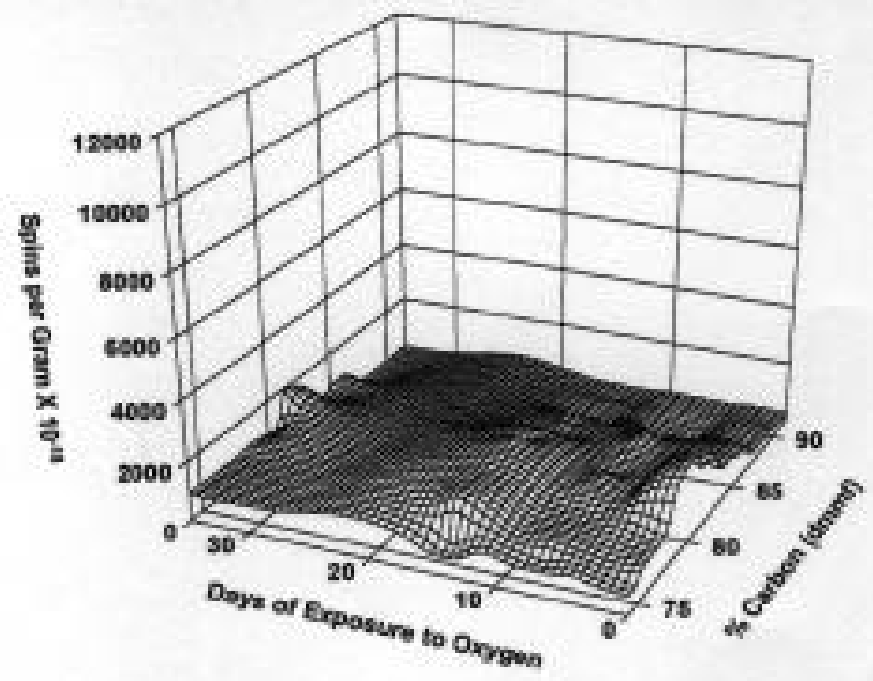

Figure 17. The retention of spin probe VI in vacuum dried coals weathered in air for up to 35 days, expressed as a function of weathering period in days of exposure, and coal rank as percent carbon. 


\section{Spin Probe VI Retention in Weathered Coals Swelled in Toluene}

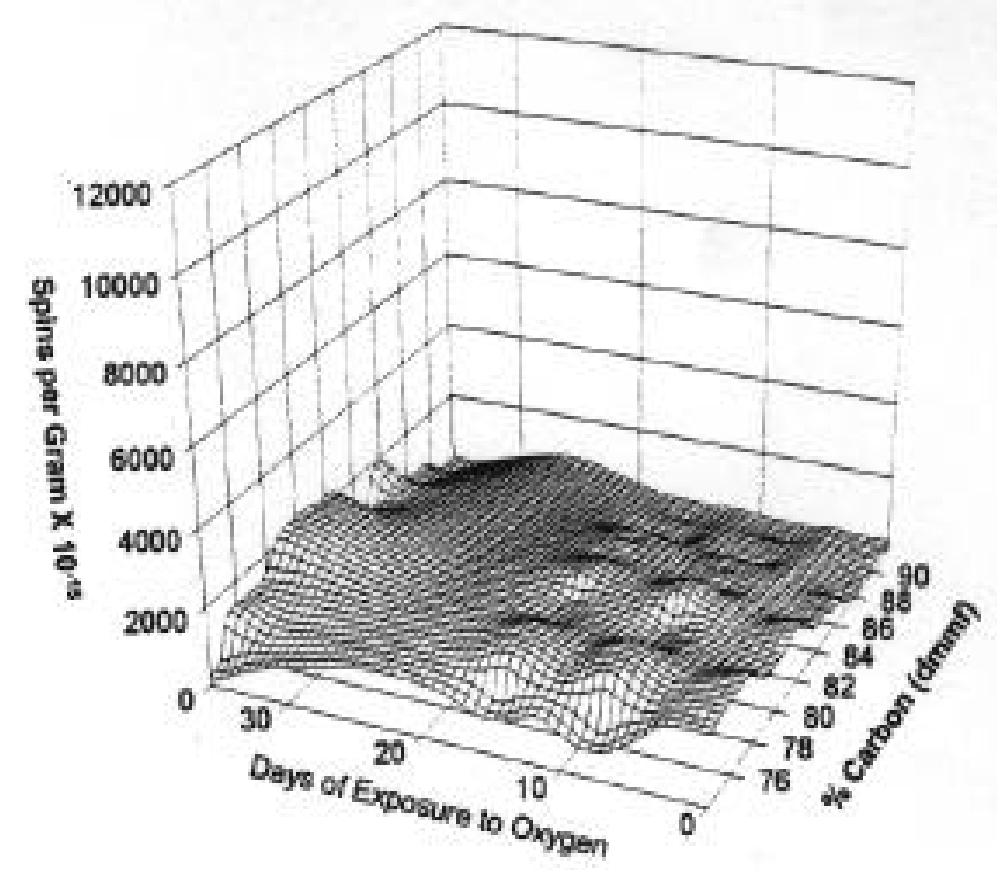

Figure 18. The retention of spin probe VI in fresh coals weathered in air for up to 36 days, expressed as a function of weathering period in days of exposure, and coal rank as percent carbon.

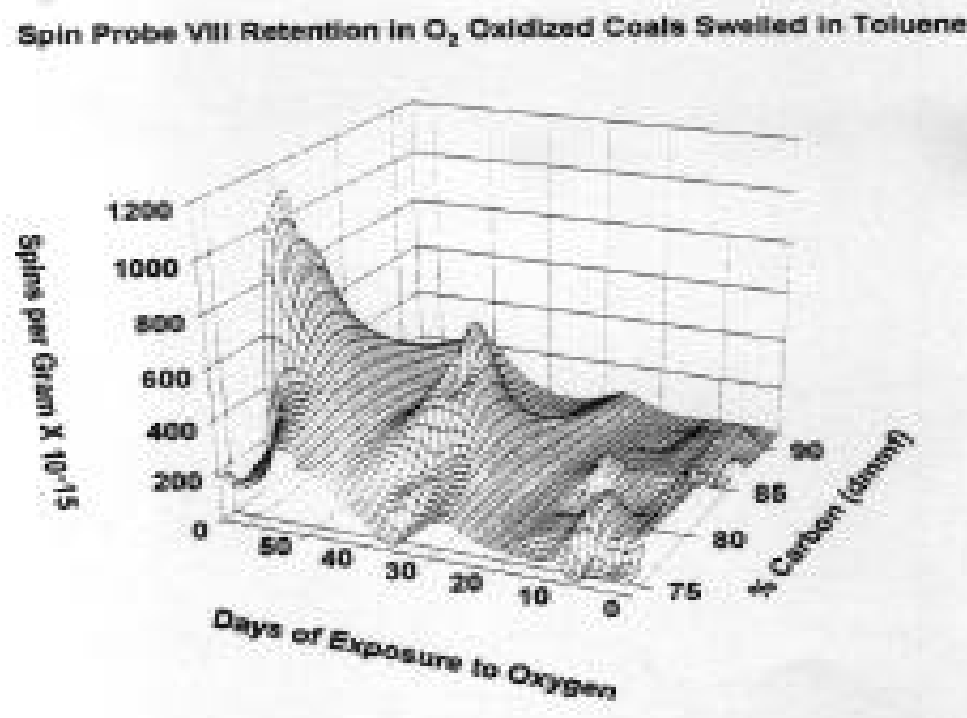

Figure 19. The retention of spin probe VI in fresh coals weathered in air for up to 64 days, expressed as a function of weathering period in days of exposure, and coal rank as percent carbon. 


\section{Spin Probe VII}

The effects of oxidation on vacuum dried coals swelled in toluene with the amino spin probe VII are plotted three dimensionally in Figure 20. The front edge parallel to the Days of Oxidation axis represents the changes undergone by Beulah-Zap $74.1 \%$ carbon dmmf) during oxidation. It is clear that the most significant changes occur within the first 15 days. At the initial exposure of 4 days, an increase in retention is seen. However, after 8 days of exposure to oxygen, Beulah-Zap exhibits a change which precludes the access of the amino spin probe to its structure. The spin probe concentration drops over an order of magnitude from $1.3 \times 10^{18}$ spins per gram at 4 days of exposure, to $7.0 \times 10^{16}$ spins per gram at 8 days of exposure shown at point $c$. At 15 days of exposure, the concentration of spin probe VII retained in Beulah-Zap increases to $1.1 \times 10^{18}$ spins per gram and does not change much as the oxidation period increases to 36 and 64 days.

Conversely, Wyodak-Anderson (76.0\% carbon dmmf) coal exhibits an initial decrease at 4 days of oxidation, followed by a larger increase at 8 days of oxidation. The spin probe concentration increased from $1.4 \times 10^{18}$ spins per gram at 4 days of oxidation to $3.3 \times 10^{18}$ spins per gram at 8 days of oxidation shown at point $b$. This increase in retention continues up to 15 days shown at point $a$, where the retention reaches a concentration of $3.9 \times 10^{18}$ spins per gram. A continued decrease is then observed until 64 days have elapsed and the spin probe concentration is lowered to $1.7 \times 10^{17}$ spin per gram.

Spin Probe VII Retention in $\mathrm{O}_{2}$ Oxidized Coals Swelled in Toluene

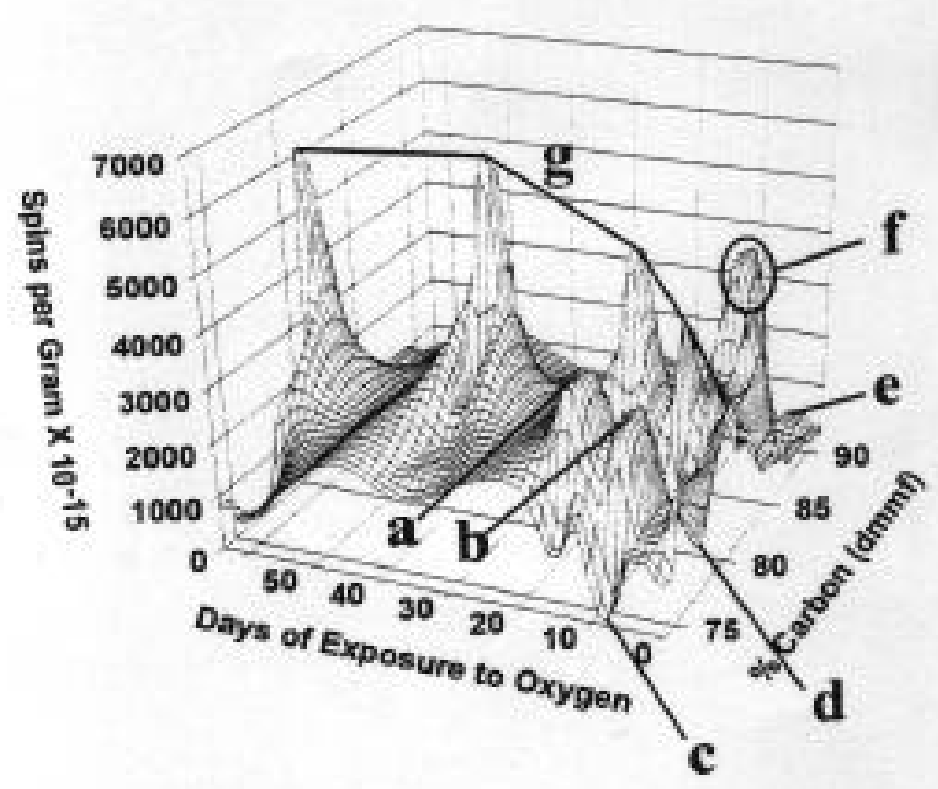

Figure 20. Retention of spin probe VII in vacuum dried APCS coals, oxidized in pure oxygen and swelled in toluene expressed in spins per gram versus days of exposure to oxygen and \% carbon (dmmf).

Illinois \#6 (80.7\% carbon dmmf) undergoes a structural change which inhibits the uptake of amino spin probes in toluene swelled coal. The spin probe retention decreases 
from an initial value of $2.9 \times 10^{18}$ spins per gram to $5.0 \times 10^{16}$ spins per gram at 4 days of exposure to oxygen and to $6 \times 10^{15}$ spins per gram at 8 days of oxidation shown at point $d$. The retention ability of Illinois \#6 oxidized beyond this point increases gradually, but not to a level anywhere near its original value. Blind Canyon (81.3\% carbon dmmf) coal behaves very differently from Illinois \#6 upon oxidation, even though both coals have similar carbon composition. The spin probe VII retention in Blind Canyon coal continually increases with oxidation until it reaches a concentration of $6.8 \times 10^{18}$ spins per gram at 36 days of oxidation. After 36 days of oxidation, very little change in retention characteristics is observed. The retention character of Blind Canyon coal is shown by the line indicated by $g$.

The high-rank coals, Pittsburgh \#8 (85.0\% carbon dmmf), Lewis Stockton (85.5^\% carbon dmmf) and Pocahontas \#3 (91.8\% carbon dmmf), all exhibit similar changes in retention characteristic during the oxidation process. After 4 days of exposure to oxygen, the vacuum dried coals all exhibit a three fold increase in spin probe VII concentration. This is shown for Pocahontas \#3 at point $e$. At 8 days of oxidation, a decrease in spin probe concentration is observed followed by an increase at 15 days. After 36 days of exposure have elapsed, the spin probe retention in Pittsburgh \#8 and Lewis Stockton coals decrease significantly, while the retention in Poccahontas \#3 actually continues to increase. There is very little change between 36 and 64 days of exposure to oxygen for any of the high-rank coals.

The previous behavior applies to all of the high-rank coals with the notable exception of Upper Freeport (88.0\% carbon dmmf). Upper Freeport coal exhibits changes in which the retention of spin probes is decreased from an initial value of $3.0 \times 10^{17}$ spins per gram to $4 \times 10^{15}$ spins per gram after 4 days of exposure to oxygen. Another dramatic change is observed between 4 and 8 days oxidation. The retention increases from $4 \times 10^{15}$ spins per gram at 4 days of oxidation to a concentration of $4.0 \times 10^{18}$ spins per gram, an increase of three orders of magnitude shown at $f$. At 15 days of oxidation, the spin probe concentration is reduced to $2.2 \times 10^{17}$ spins per gram. Beyond 15 days, the spin probe concentration increases with oxidation up to 64 days.

\section{Spin Probe VIII}

The changes in the ability of coals to intercalate the unsubstituted spin probe VIII over an oxidation period of 64 days is shown by the three dimensional plot in Figure 21. Again looking at the front edge parallel to the Days of Exposure to Oxygen axis, it can be seen that the spin probe concentration is reduced significantly at 8 days of exposure to oxygen. At this point the spin probe VIII concentration is reduced to $2 \times 10^{16}$ spins per gram. As the oxidation period increases beyond 8 days, the spin probe concentration increases until at 64 days it has increased an order of magnitude to $2 \times 10^{17}$ spins per gram.

Both of the medium ranked coals (Illinois \#6 and Blind Canyon) exhibit similar behavior in the retention of spin probe VIII upon oxidation. As the oxidation period increases, the concentration of spin probe VIII in the medium ranked coals increases until at 64 days they are at 5 times their initial value. 


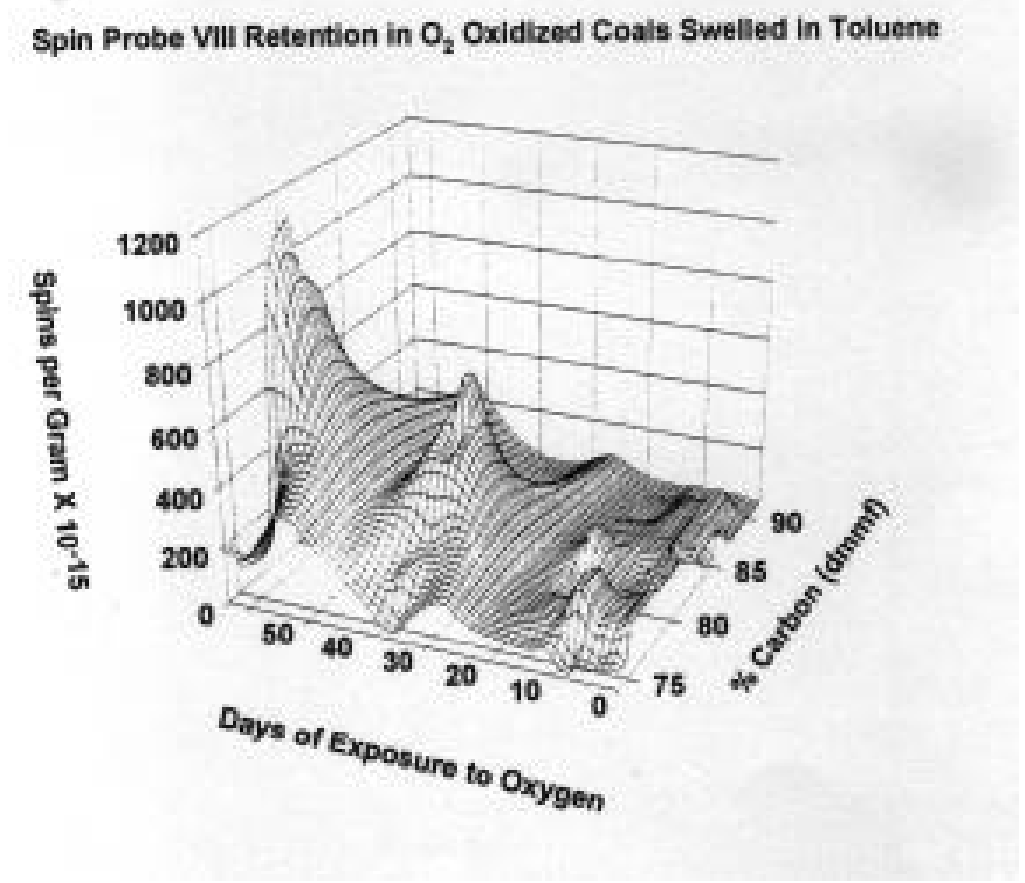

Figure 21. Retention of spin probe VIII in vacuum dried APCS coals, oxidized in pure oxygen and swelled in toluene, expressed in spins per gram versus days of exposure to oxygen and \% carbon (dmmf).

The higher ranked are observed to have similar retention characteristics during oxidation. They all show an increase in spin probe retention at 4 days of oxidation, followed by a decrease at 15 days and a continuous increase in retention thereafter, until at 64 days of exposure, they retain concentrations of spin probe VIII over an order of magnitude higher than their original values.

\section{Discussion}

The results observed for the retention of spin probes VI and VII in oxidized coals swelled in toluene are very similar. It is observed that oxidation can produce changes in the structure of high-rank coals so that large amounts of polar probes can be intercalated in the coal structure. This is partially due to the increase in the number of active sites capable of hydrogen bond interactions. However, since there was a similar increase observed for the retention of spin probe VIII, which does not exhibit polar interaction with the coal structure, high-rank coals must undergo changes in their physical structure during oxidation which allow additional access to the smaller spin probes. This indicates that the covalent cross-links in high-rank coals are disrupted by the oxidation process.

Because a large increase in spin probe VIII retention is observed for medium ranked coals during the oxidation process, it is clear that oxidation causes an opening of the structure in these coals which is responsible for much of the observed behavior for all of the spin probes. It should be noted that the changes brought about by oxidation which cause large differences in the retention of the polar spin probes in Illinois \#6 and Blind Canyon coals must be due to unequal changes in the hydrogen bonded character of these 
coals. The retention character of all three spin probes in toluene swelled WyodakAnderson coal is very similar. Since it has been previously shown that Wyodak-Anderson coal undergoes a partial collapse of structure upon vacuum drying, it can be seen that at 8 days of exposure the oxidation process reopens the structure so that the spin probes can regain accessibility to the coal.

Since a decrease in retention is observed for all three spin probes in toluene swelled Beulah-Zap lignite at 8 days of exposure to oxygen, it is clear that the physical structure must undergo a collapse which would preclude the intercalation of spin probes. This suggests that oxidation can also play an important role in the structural collapse of coals during the weathering process. Continued exposure of Beulah-Zap lignite to oxygen, reopens the structure so that significant amounts of spin probes can be retained. As the oxidation period is increased from 36 to 64 days, a drop in spin probe VII (a hydrogen bond acceptor) retention is observed and large increase in spin probe VI (a hydrogen bond donor) is observed. This indicates that the chemical changes observed in Beulah-Zap upon oxidation at 64 days include a large increase in the number of hydrogen bond acceptor sites and, to a lessor extent, a corresponding decrease in the number of hydrogen bond donor sites.

These results are compared to the results obtained for weathered coals swelled in toluene for the case of spin probe VI (Figures 15 and 19) in Figure 22. The plots are set to the same scale and shown side by side for ease of comparison. It can be seen that oxidation can effect the structure of coal to a much larger extent than just weathering. This would suggest that the changes caused by oxidation are somewhat inhibited by the presence of moisture.
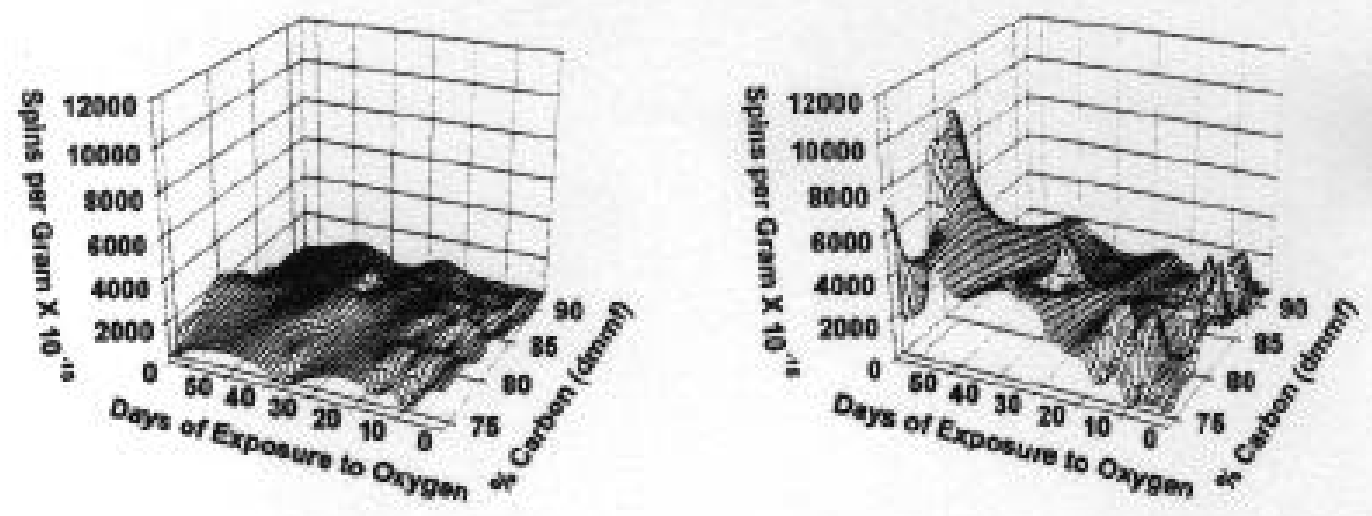

Figure 22. A comparison of the retention of spin probe VI in coals weathered in air (on the left) and coals oxidized in $\mathrm{O}_{2}$ (on the right). Toluene was used as the swelling solvent.

\section{Oxidation Of Individual Vacuum Dried Coals}

\section{Beulah Zap Lignite (74.1\% carbon dmmf)}

In Figure 23, the retention of spin probes VI, VII and VIII in vacuum dried Beulah Zap lignite swelled in both pyridine and toluene is expressed as a function of oxidation period. The coals were all exposed to dry oxygen at $0 \%$ humidity. In the case of coal 
swelled in pyridine, the data points are marked by solid figures, and in the case of toluene, the data points are marked by open figures. Spin probe VI is represented by circles, spin probe VII by squares and spin probe VIII by triangles. The retention data was scaled logarithmically so that all of the spin probes could be plotted on the same graph.

Likewise, some of the more significant changes are easier to see. It is important to note that only relative changes in spin probe retention are being discussed at each oxidation period, and a quantitative analysis of the data is not attempted or relevant to the discussion. Lines are drawn through data only so that changes in retention character during oxidation can be more easily observed.

The retention data at 0 days represents the case for vacuum dried coals immediately swelled in the respective spin probe solution.

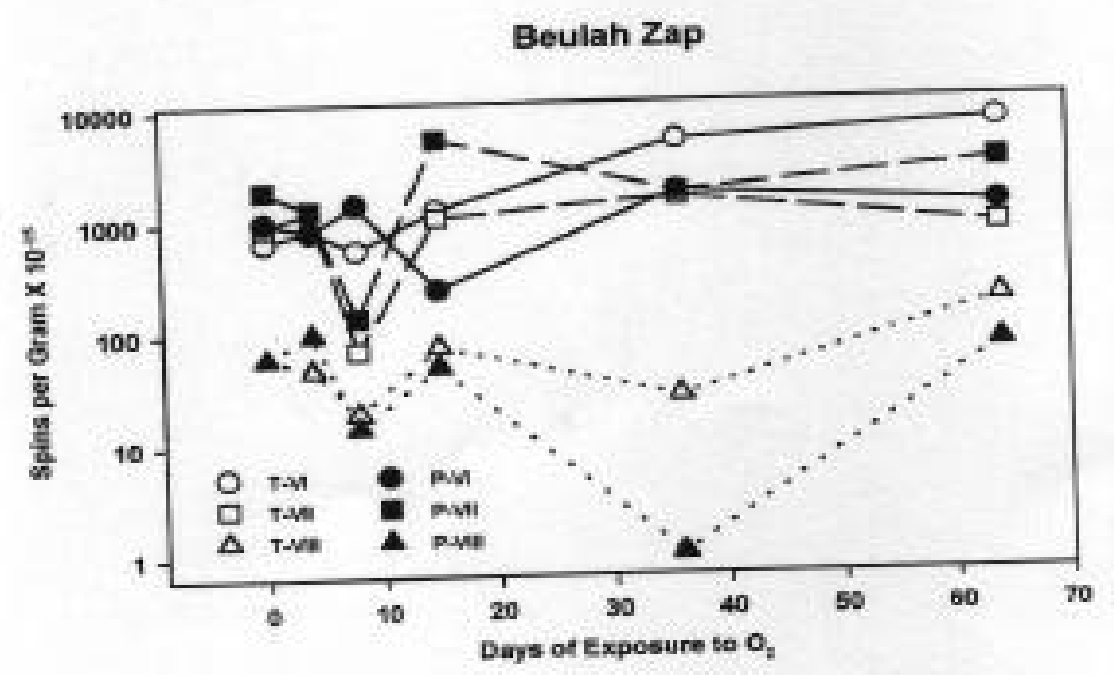

Figure 23. The retention of spin probes VI (O), VII $(\square)$ and VIII $(\Delta)$,- in vacuum dried Beulah Zap lignite swelled in toluene (open symbols) and pyridine (closed symbols) as a function of oxidation period expressed in days.

After 4 days of exposure to oxygen, both of the polar spin probes swelled in toluene exhibit an increase in retention, and the polar spin probes swelled in pyridine exhibit a decrease in retention. The relative magnitude of these changes is about the same for all 4 cases. A similar change is observed for the retention of spin probe VIII swelled in toluene and pyridine. A decrease in retention is observed for spin probe VIII in toluene and an increase in retention of spin probe VIII in pyridine. The difference in the retention changes of spin probes in coal swelled in pyridine and toluene indicates some kind of change in the hydrogen bonded character of the coal structure since pyridine disrupts these hydrogen bonds and toluene does not. 
At 8 days of exposure, there is a significant decrease in the retention of spin probe VIII in both pyridine and toluene swelled Beulah Zap lignite, indicating a change in the covalent cross-linking in coal. Likewise a decrease in the retention of spin probe VII in both pyridine and toluene swelled lignite is observed. This is caused by an increase in the covalent cross-linking which limits the accessibility since pyridine does not break down these cross-links but does disrupt any hydrogen bond interactions. Spin probe VI retention, however, shows only a slight decrease in toluene and an increase in pyridine. This indicates that some of the $\mathrm{OH}$ groups are being converted to $\mathrm{C}=\mathrm{O}$ since no similar changes in the concentration of spin probe VII were observed. It can also be seen that these new functionalities are involved in hydrogen bond interactions since more of spin probe VI is retained the case of lignite swelled in pyridine.

As the oxidation period is extended to 15 days, a strong increase is observed for spin probe VIII in lignite swelled in both toluene and pyridine. This could be caused by a physical rearranging of the lignite structure which does not involve hydrogen bond interactions or disruption of some of the recently formed covalent cross-links. In the second case, the cross-links being disrupted would most likely consist of oxidation of peroxide linkages. To a larger extent, there is an observed increase in spin probe VII in both toluene and pyridine swelled lignite. This would support a mechanism where the peroxide linkages could be oxidize to $\mathrm{COOH}$, and since the increase for spin probe VI in pyridine is so much greater, some of the $\mathrm{C}=\mathrm{O}$ and $\mathrm{OH}$ groups might also be converted to $\mathrm{COOH}$. The slight increase in the retention of spin probe VI in lignite swelled in toluene is most likely due strictly to the opening of the accessibility of the small spin probes. This is further supported since the retention of spin probe VI in pyridine swelled lignite is actually decreased. This also indicates that the $\mathrm{C}=\mathrm{O}$ groups previously formed are being converted to hydrogen bond donors like $\mathrm{COOH}$.

At 36 days, the retention of spin probe VIII swelled in pyridine is decreased significantly while only slightly when toluene is used. This indicates that the structure was opened to such an extent that the spin probe is being washed out. The decrease in the retention of spin probe VIII in toluene indicates that there is a significant increase in hydrogen bond cross-linking which is disrupted by pyridine but not significantly altered by toluene. The decrease in retention of spin probe VII in pyridine swelled lignite can be explained if some of the previously formed $\mathrm{COOH}$ groups are now involved in polar crosslink interactions. For spin probe VI there is an observed increase for both pyridine and toluene swelled lignite. This would indicate that more $\mathrm{OH}$ or $\mathrm{C}=\mathrm{O}$ groups are being formed at this point.

After 64 days of exposure to oxygen, a large increase in the retention of both toluene and pyridine swelled coal is observed. This indicates that the physical structure is changed such that more accessible regions are being formed. This could be caused by a partial closing of the structure, or more likely, oxidative degradation of new unsaturated sections of the lignite. This newly opened area would then be subject to further oxidation and there would then be another oscillation in retention behavior.

\section{Wyodak Anderson Coal $(76.0 \%$ carbon dmmf)}

In Figure 24, the retention of spin probes VI, VII and VIII in vacuum dried Wyodak Anderson coal swelled in both pyridine and toluene is expressed as a function of oxidation period. The coals were all exposed to dry oxygen at $0 \%$ humidity. In the case of coal swelled in pyridine, the data points are marked by solid figures, and in the case of toluene, the data points are marked by open figures. Spin probe VI is represented by circles, spin probe VII by squares and spin probe VIII by triangles. The retention data was 
scaled logarithmically so that all of the spin probes could be plotted on the same graph. Likewise, some of the more significant changes are easier to see.

After four days of exposure to oxygen, a slight decrease in the retention of spin probe VIII in toluene swelled Wyodak Anderson coal is observed. Also, the retention of spin probe VIII pyridine swelled coal is increased. These results are very similar to those seen for Beulah Zap lignite. Pyridine breaks up the hydrogen bonding in the coal structure, and so the increase in retention is indicative of an increase in small accessible regions. Although small accessible regions are being created, a decrease is observed in the case of toluene swelled Illinois \#6 coal.

Wyodak Anderson

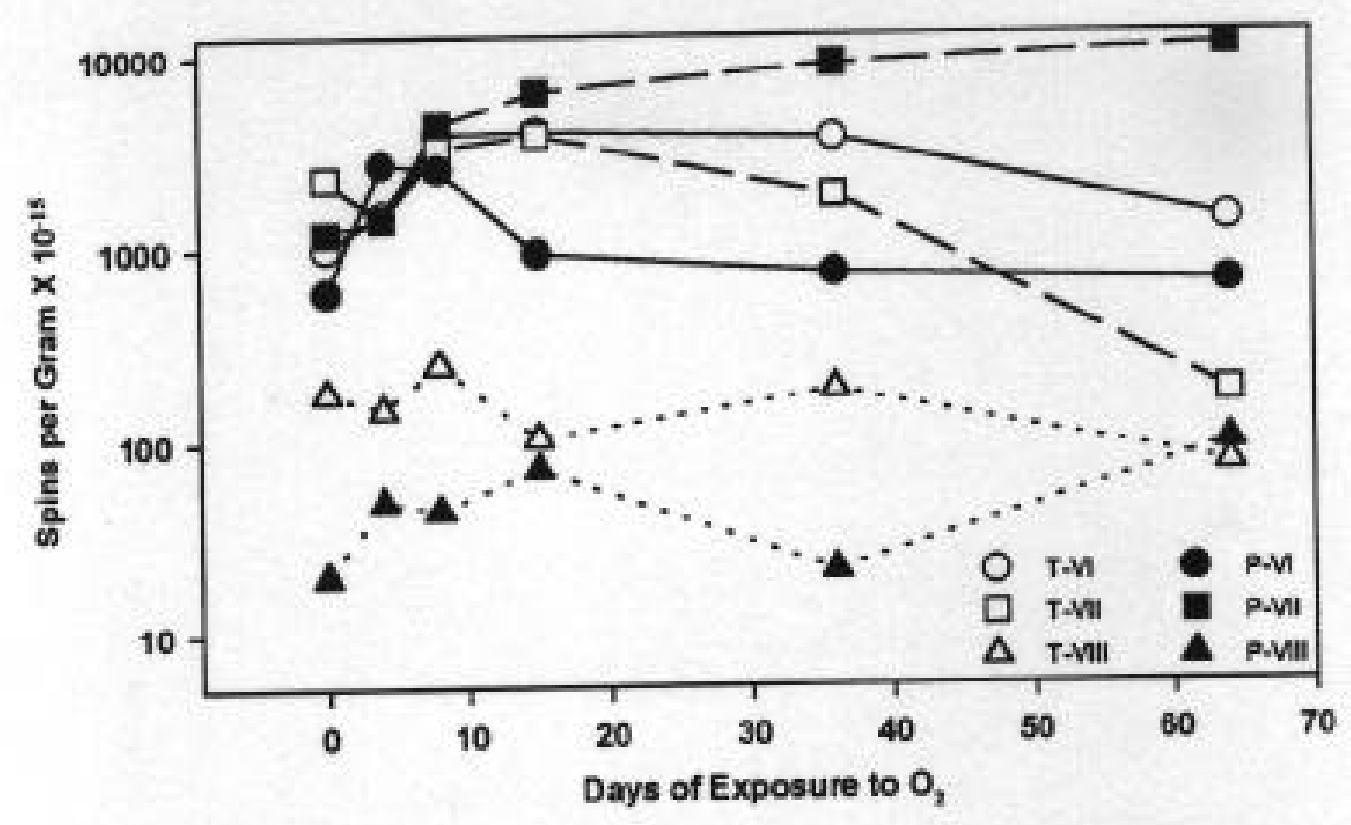

Figure 24. The retention of spin probes VI (O), VII $(\square)$ and VIII $(\Delta)$ in vacuum dried Wyodak Anderson coal swelled in toluene (open symbols) and pyridine (closed symbols) as a function of oxidation period expressed in days.

A change in the hydrogen bonded structure is indicated. Since the retention in toluene decreased while the retention in pyridine increased, it is most likely that while more small pores or accessible areas were being formed, an increase in hydrogen bond crosslinking took place. Also, since the increase observed for pyridine is so significant, it is likely that some of the covalent cross-linking has broken down. Looking at spin probe VII retained in toluene and pyridine swelled coal, a decrease is observed in the case of toluene and a slight increase is observed in the case of pyridine. This is an indication that hydrogen bonding was indeed increased enough so that limited access was observed for toluene swelled coal. Also the immediately accessible region exhibited some conversion of $\mathrm{C}-\mathrm{OH}$ to $\mathrm{C}=\mathrm{O}$, as well as the formation of new $\mathrm{OH}$. This can be verified by the retention behavior observed of spin probe VI in toluene an pyridine swelled coal. A slight increase is observed for the case of toluene, where a larger increase is observed in the case of pyridine. Again this shows that some of the $\mathrm{C}-\mathrm{OH}$ concentration in the coal is being converted to $\mathrm{C}=\mathrm{O}$. However, a large percentage of this change is not available in the toluene swelled coal, presumably due to increased hydrogen bonding. 
As the oxidation period is increased to 8 days, an increase in retention is observed for spin probe VIII in toluene swelled coal, but no real change is observed for pyridine swelled coal. This would indicate an opening of the hydrogen bonded structure to the smaller pores, and very little change in the covalently bonded structure. A significant increase in spin probe retention is observed for all of the polar spin probes except for the case of spin probe VI retained in pyridine swelled coal. Some of the increase observed for the toluene swelled coal must be due to the opening of the hydrogen bonded structure, as shown by the retention behavior of spin probe VIII. It is likely that more $\mathrm{OH}$ is being formed, especially in the immediately accessible regions, but that some of the previously formed $\mathrm{C}=\mathrm{O}$ is undergoing further oxidation to $\mathrm{COOH}$, since very little change was observed for spin probe VI in pyridine.

After the Wyodak Anderson coal has been exposed to oxygen for 15 days, an increase in the retention of spin probe VIII in pyridine swelled coal is observed, and a decrease in spin probe VIII retention in toluene swelled coal is observed. This is an indication that the oxygen species being formed are causing an increase in the hydrogen bonded structure. Also, since an increase in retention is observed for the case of pyridine, an increase in porous structure availability must be due to further attack of the covalent structure of the coal by oxidation. Very little change is observed for the retention of the polar spin probes in toluene swelled coal. Since spin probe VIII retention has shown an increase in hydrogen bonded structure, some new $\mathrm{OH}$ groups must be formed in the immediately accessible areas to offset the decrease in accessibility to the microporous regions. This is also supported by the observed disruption of the covalent cross-links. Looking at the retention behavior of the polar spin probes in pyridine swelled coal, it is seen that there is a strong decrease for spin probe VI and an increase for spin probe VII. This is a clear indication that hydrogen bond acceptors are being reduced, while hydrogen bond donors are increasing in concentration. Much of the $\mathrm{C}=\mathrm{O}$ is probably being oxidized to $\mathrm{COOH}$.

At 36 days of exposure, the retention of spin probe VIII decreases in pyridine swelled coal but increases in toluene swelled coal. This indicates further attack of the covalent structure such that the coal is opened too much for significant retention of the small unsubstituted spin probe in pyridine. While virtually no change is observed for the retention of spin probe VI in coal swelled in either toluene or pyridine, spin probe VII retention increases in pyridine and decreases in toluene swelled coal. This could result from the formation of additional polar functionalities which interact with spin probe VII in pyridine swelled coal, but are involved in hydrogen bonding and are therefore not detected in the case of toluene swelled coal. However, since the retention of spin probe VIII in toluene swelled coal was not inhibited, it is a clear that the oxidation process must be diffusion related. The areas accessible to toluene are more accessible to the oxygen environment, and clearly undergo changes which are different from the changes observed for the overall coal structure. Since the retention of spin probe VII increases in pyridine swelled coal, there is an overall increase in hydrogen bond donor sites resulting from conversion of $\mathrm{C}=\mathrm{O}$ and $\mathrm{OH}$ to $\mathrm{COOH}$.

After 64 days have passed, spin probe VIII retention exhibits a large increase in pyridine swelled Wyodak Anderson coal and a decrease in toluene swelled coal. This again indicates that further degradation of the covalent structure of coal is occurring. This degradation results in new active sites which are in close enough proximity to form hydrogen bonded cross-linking which inhibits the retention of spin probe VIII in toluene swelled coal. Both of the polar spin probes show a decrease in retention in toluene swelled coal. This decrease is more significant for spin probe VII. The observed decrease of spin probe VI in toluene is similar to that of spin probe VIII, indicating that the increase in noncovalent cross-linking is the primary reason for the change. However, the larger change 
observed by spin probe VII indicates a reduction of $\mathrm{OH}$ and an increase in $\mathrm{C}=\mathrm{O}$ groups. Looking at the retention character of pyridine swelled coals, it is easy to see that the chemical changes exhibited by the over-all coal are much different that those limited by the hydrogen bonding. The increase of spin probe VII in pyridine swelled coal indicates that there is an overall increase in $\mathrm{COOH}$.

\section{Illinois \#6 Coal $(80.7 \%$ carbon dmmf)}

In Figure 25, the retention of spin probes VI, VII and VIII in vacuum dried Illinois \#6 coal swelled in both pyridine and toluene is expressed as a function of oxidation period. The coals were all exposed to dry oxygen at $0 \%$ humidity. In the case of coal swelled in pyridine, the data points are marked by solid figures, and in the case of toluene, the data points are marked by open figures. Spin probe VI is represented by circles, spin probe VII by squares and spin probe VIII by triangles. The retention data was scaled logarithmically so that all of the spin probes could be plotted on the same graph. Likewise, some of the more significant changes are easier to see.

Initially there is very little difference in the retention of either of the polar spin probes in toluene swelled Illinois \#6 coal. However, as four days of oxidation have passed, both of these spin probes exhibit a significant decrease in retention. This decrease is much larger for spin probe VII than for spin probe VI. The stronger decrease in toluene swelled coal indicates that the hydrogen bonded structure has increased in cross-linking. The strong decrease observed by spin probe VII to such low level would indicate that extensive hydrogen bonded cross-linking had occurred, and that during the first four days, formation of $\mathrm{C}=\mathrm{O}$ is as significant as the formation of $\mathrm{OH}$ groups. This is further supported by the observed increase in the retention of spin probe VI in pyridine swelled coal, where the hydrogen bonded structure is disrupted. Also, the reduction of spin probe VIII in pyridine swelled coal provides indication that termination of radicals formed during oxidation process is increasing the covalent cross-linking, which further inhibits the accessibility of spin probes even though new oxygenated functional groups are being formed. 


\section{Illinois \#6}

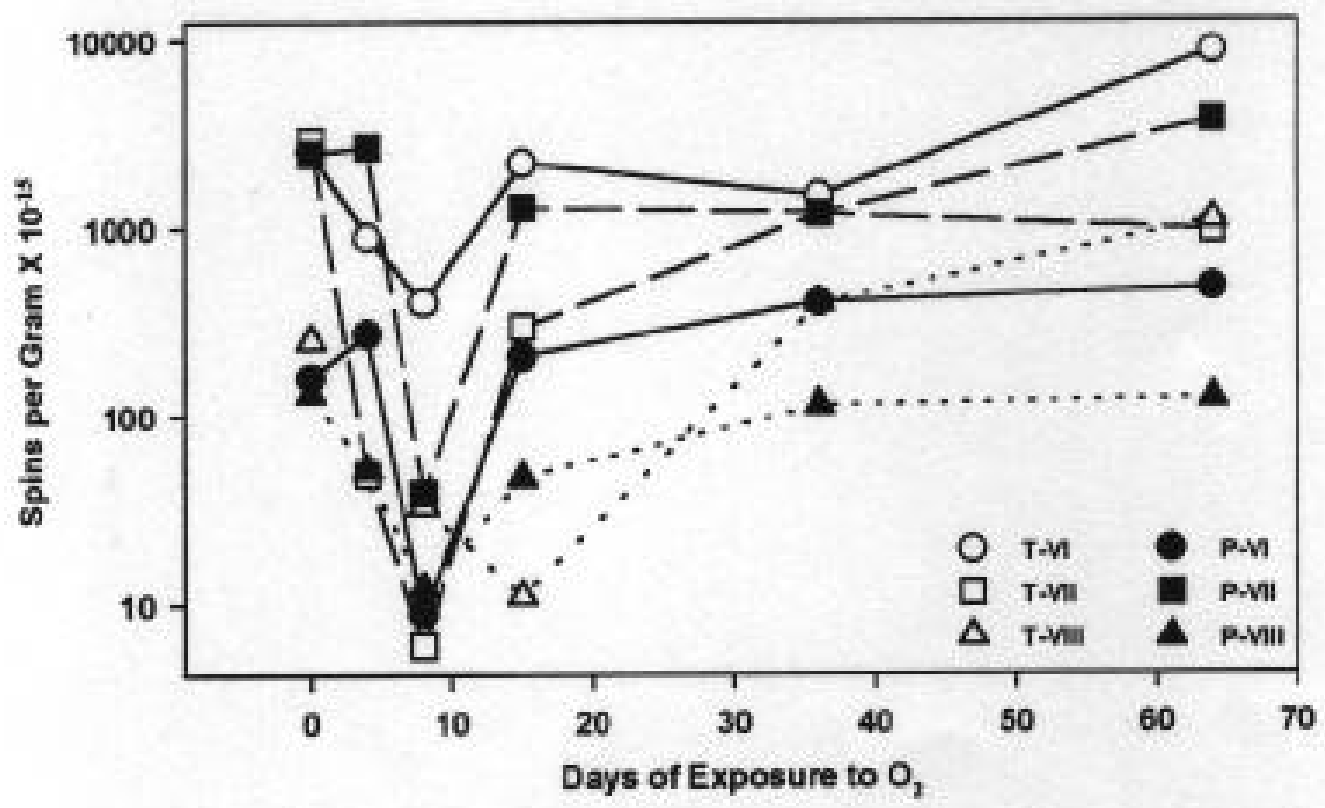

Figure 25. The retention of spin probes VI (O), VII $(\square)$ and VIII $(\Delta)$ in vacuum dried Illinois \#6 coal swelled in toluene (open symbols) and pyridine (closed symbols) as a function of oxidation.

At eight days of exposure to oxygen, a strong decrease is observed for all three spin probes in Illinois \#6 coal swelled in both toluene and pyridine. The strong decrease in all of the spin probe concentrations in pyridine swelled coal indicates the establishment of further covalent cross-linking, which inhibits the accessibility of the spin probes. It is interesting to note that the concentration of spin probe VI in pyridine swelled coal actually drops below the concentration of spin probe VIII. Although the difference is very small, it does indicate that the carboxyl group is providing no additional support in the intercalation process. The same is observed for spin probe VII in toluene swelled coal but to a much greater extent. In this case, the concentration of spin probe VII drops significantly below the concentration of spin probe VIII. Since the sizes of spin probes VII and VIII are similar, the changes in the covalent structure must have created non-polar regions in the structure of the coal not blocked off by hydrogen bonding. Because the concentration of spin probe VI in toluene swelled coal did not drop as drastically, it can be asserted that much of the $\mathrm{OH}$ functionality must have been converted to hydrogen bond acceptor groups like $\mathrm{C}=\mathrm{O}$. If this was not the case, more of spin probe VII would have been retained. It is important to note that these changes occur only in the immediately accessible areas where hydrogen bonded character cannot inhibit the access of spin probes to the structure.

At 15 days of exposure to oxygen, all of the spin probe concentrations increase with the exception of spin probe VIII in toluene swelled coal. Because the concentration of spin probe VIII decreases in toluene swelled coal, it is likely that a significant increase in hydrogen bonded cross-linking has occurred. This is offset by the increase in polar functional groups capable of exhibiting hydrogen bond interactions for spin probes VI and VII. Because there is an increase for both of the polar spin probes, it is likely that the majority of new functionalities consist of $\mathrm{OH}$ groups which act as both hydrogen bond donors and acceptors. Also, since all of the polar spin probes show stronger retention than 
the unsubstituted spin probe, at this point, the non-polar pores which had previously formed have most likely undergone oxidation. With the observed increase in the concentration of the other spin probes, it is likely that the increase in retention observed for spin probe VIII in pyridine swelled Illinois \#6 coal is due to oxidative degradation of the covalent structure. It is apparent that the changes in the coal structure during oxidation are different for areas which are accessible by spin probes inhibited by hydrogen bond interactions, and those which are accessible by spin probes limited only by covalent interactions. For the case of toluene swelled coal, the retention of spin probe VI exceeds that of spin probe VII, indicating an environment of increased hydrogen bond acceptor sites. However, in pyridine swelled coal, the concentration of spin probe VII exceeds that of spin probe VI. Especially since pyridine itself competes with spin probe VII for hydrogen bond donor sites, it is evident that the structure opened by pyridine has a higher concentration of hydrogen bond donor sites.

After 36 days of exposure to the oxygen environment, the concentration of spin probe VIII in both toluene and pyridine swelled coal was increased. The increase observed for spin probe VIII in pyridine swelled coal indicates a further opening of the coal structure to smaller micropores. The more drastic increase observed by spin probe VIII in toluene swelled coals indicates that the hydrogen bonded structure has been opened to a significant extent. The retention of the polar spin probes in pyridine swelled coal remains relatively unchanged. Spin probe VI retention increases in pyridine swelled coal, but not to any extent more than the increase in the accessible regions indicated by spin probe VIII. However, in toluene swelled coal, an increase is observed for the retention of spin probe VII and a slight increase in retention is observed for spin probe VI. This indicates that in the more accessible regions of the coal, conversion of $\mathrm{C}=\mathrm{O}$ to $\mathrm{COOH}$ is taking place.

The changes observed as the coal is exposed to oxygen for 64 days are less intense than any of the other changes observed previously. A slight increase in retention is observed for spin probe VIII in pyridine swelled coal. Some additional micropore pore regions could be formed at this point, but the change is not very significant. In toluene swelled coal, however, a significant increase in spin probe VIII retention does occur. Again the hydrogen bonded structure is further altered to allow accessibility in toluene swelled coal. The retention of both polar spin probes in pyridine swelled coal exhibit an increase. The retention of spin probe VI in pyridine swelled coal increases only slightly in a manner very similar to spin probe VIII. It is likely that this increase is simply due to an increase in accessible areas. Spin probe VII exhibits a more significant increase however, indicating that more hydrogen bond donor groups $(\mathrm{COOH})$ are being generated. The reverse seems to be true for toluene swelled coal. Spin probe VII shows very little change while spin probe VI increases in concentration. The concentration of spin probe VII is about the same as spin probe VIII in toluene swelled coal. This is indication that there are more non-polar pores being formedwhich allow further inclusion of spin probe VIII without affecting the retention of the polar spin probe to any large extent. The increase in spin probe VI retention in toluene swelled coal indicates that further conversion of $\mathrm{OH}$ to $\mathrm{C}=\mathrm{O}$ is taking place. It is also possible that the slight decrease in retention of spin probe VII could be accounted for by some of the $\mathrm{COOH}$ further oxidizing to $\mathrm{CO}_{2}$ in these regions.

\section{Blind Canyon Coal (81.3\% carbon dmmf)}

In Figure 26, the retention of spin probes VI, VII and VIII in vacuum dried Blind Canyon coal swelled in both pyridine and toluene is expressed as a function of oxidation period. The coals were all exposed to dry oxygen at $0 \%$ humidity. In the case of coal swelled in pyridine, the data points are marked by solid figures, and in the case of toluene, the data points are marked by open figures. Spin probe VI is represented by circles, spin 
probe VII by squares and spin probe VIII by triangles. The retention data was scaled logarithmically so that all of the spin probes could be plotted on the same graph.

After Blind Canyon coal has been exposed to oxygen for 4 days, the retention of spin probe VIII decreases significantly in toluene swelled coal and increases in pyridine swelled coal. This indicates that even though the structure of the coal is opened up, the hydrogen bonded cross-links are increased, inhibiting the access of spin probes in toluene swelled coal. A strong increase in the retention of both polar spin probes is observed in pyridine swelled coal. This indicates a significant increase in the concentration of $\mathrm{OH}$ groups. In toluene swelled coal, spin probe VI retention remains relatively unchanged while the concentration of spin probe VII increases slightly. The observed increase in hydrogen bonded cross-linking probably offsets the increase in $\mathrm{OH}$ concentration so that the retention characteristics of the polar spin probes in toluene are affected to a lesser extent.

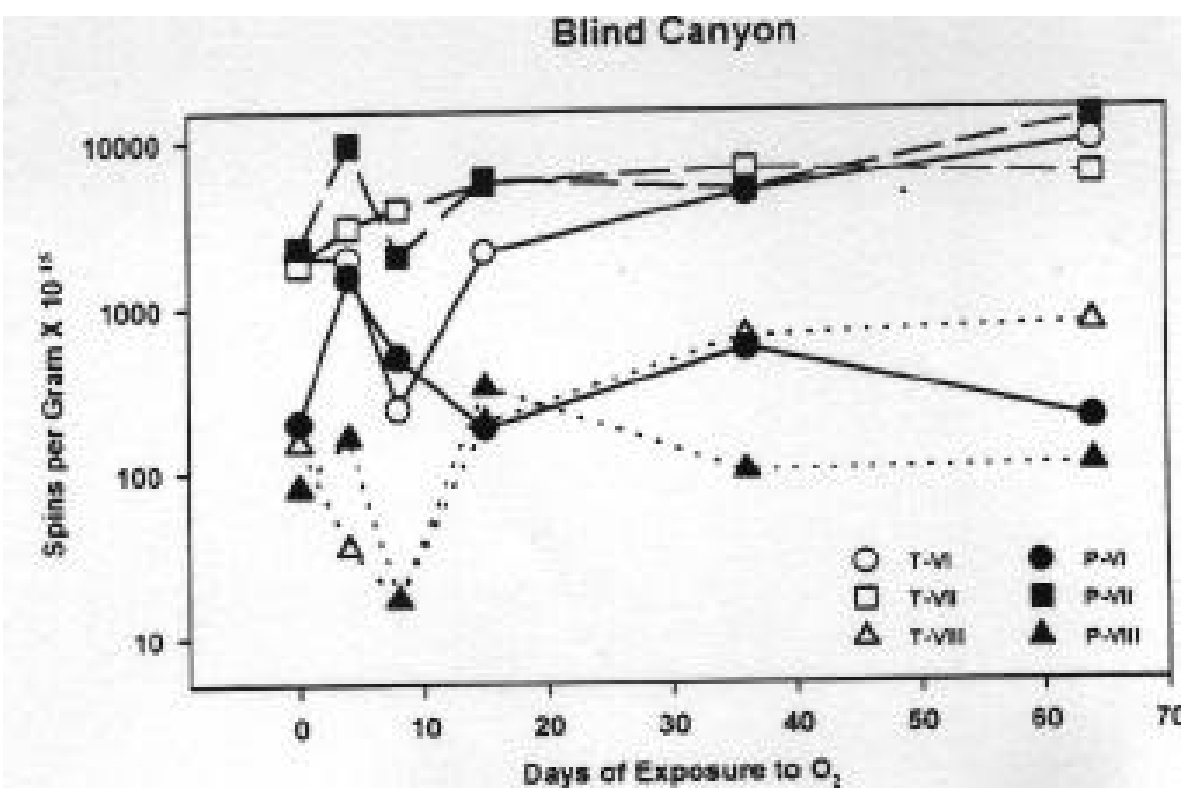

Figure 26. The retention of spin probes VI (O), VII $(\square)$ and VIII $(\Delta)$ in vacuum dried Blind Canyon coal swelled in toluene (open symbols) and pyridine (closed symbols) as a function of oxidation period expressed in days.

At 8 days of exposure, the retention of all the spin probes is sharply decreased with the exception of spin probe VII in toluene swelled coal, which increases slightly. The strong decrease exhibited by spin probe VIII in both toluene and pyridine swelled coal indicates a collapse of the structure or an increase in the covalent cross-linking to such an extent that access to the micropore structure is inhibited. The increased covalent crosslinking is also responsible for the decrease in accessibility of the other spin probes. Because the concentration of spin probe VII in both toluene and pyridine swelled coal remains much higher than the others, it is likely that some $\mathrm{COOH}$ is being formed in the structure at this point.

As the exposure period to oxygen is increased to 15 days, the concentration of all of the spin probes is increased with the exception of spin probe VI in pyridine swelled coal, which decreases. The concentration of spin probe VIII increases sharply in both toluene and pyridine swelled Blind Canyon coal. This indicates that the covalent cross-links in the coal structure have been attacked and the structure has opened up and become accessible to the small spin probes. Although both of the polar spin probe exhibit an increase in 
retention in toluene swelled coal, the increase in spin probe VII is strong while the increase in spin probe VI is fairly small. Since the structure has opened to some extent, and an increase would be expected for the polar spin probes, it is reasonable to suggest that some of the $\mathrm{OH}$ are being converted to $\mathrm{C}=\mathrm{O}$, accounting for the disproportionate increase in spin probe VI retention. Looking at the retention of the polar spin probes in pyridine swelled coal, it is clear that a larger percentage of $\mathrm{COOH}$ is present, and that these groups are either involved in hydrogen bonding or protected by hydrogen bonding. The retention in pyridine swelled coal increases in the case of spin probe VII and just as strongly decreases for spin probe VI, indicating an overall increase in hydrogen bond donor sites and an overall decrease in hydrogen bond acceptor sites. Because the concentration on spin probe VI is less than spin probe VIII in pyridine swelled coal, it is likely that some non-polar pores have been formed at this point.

The retention of spin probe VIII in pyridine swelled coal decreases upon increasing the period of oxidation to 36 days. Since other spin probes show increases in retention, the decrease would have to be accounted for by further degradation of the covalent crosslinking and the reduction of non-polar pores in the structure. The structure opens enough so that the unsubstituted spin probe is washed out after swelling with pyridine. The retention of spin probe VIII in toluene swelled coal, however, increases. This also supports the opening of the covalent structure as well as the hydrogen bonded structure. The concentration of spin probe VI increases in both toluene and pyridine swelled coal. This indicates an increase the concentration of $\mathrm{C}=\mathrm{O}$ groups in the coal structure. Also, the increase in concentration of spin probe VI in pyridine swelled coal to a level far greater than spin probe VIII indicates that the non-polar pores have been oxidized. Spin probe VII exhibits very little change in either toluene or pyridine swelled coal.

At 64 days of exposure, the changes in retention character are not as drastic as during the first 15 days of oxidation. The retention of spin probe VIII in both toluene and pyridine swelled coal remains relatively unchanged with only a very slight increase in both cases. This suggests that the physical structure does not change very much during this period. Likewise, spin probe VII retention in toluene swelled coal does not change much, although spin probe VI retention in toluene swelled coal does increase slightly. This indicates that, in the more accessible region not blocked by hydrogen bond interactions, additional formation of $\mathrm{C}=\mathrm{O}$ has occurred. In pyridine swelled coal it is seen that the retention of spin probe VII increases while the retention of spin probe VI decreases. Therefore, in the coal structure limited only by covalent interactions there must be a reduction of $\mathrm{C}=\mathrm{O}$ and an increase in $\mathrm{COOH}$.

Blind Canyon coal exhibits an increase in covalent cross-linking, followed by an attack on that cross-linking which results in an increase in both $\mathrm{C}=\mathrm{O}$ and $\mathrm{COOH}$ group.

\section{Lewiston-Stockton Coal $(85.0 \%$ carbon dmmf)}

In Figure 27, the retention of spin probes VI, VII and VIII in vacuum dried Lewiston-Stockton coal swelled in both pyridine and toluene is expressed as a function of oxidation period. The coals were all exposed to dry oxygen at $0 \%$ humidity. In the case of coal swelled in pyridine, the data points are marked by solid figures, and in the case of toluene, the data points are marked by open figures. Spin probe VI is represented by circles, spin probe VII by squares and spin probe VIII by triangles. The retention data was scaled logarithmically so that all of the spin probes could be plotted on the same graph. Also, some of the more significant changes are easier to see. 


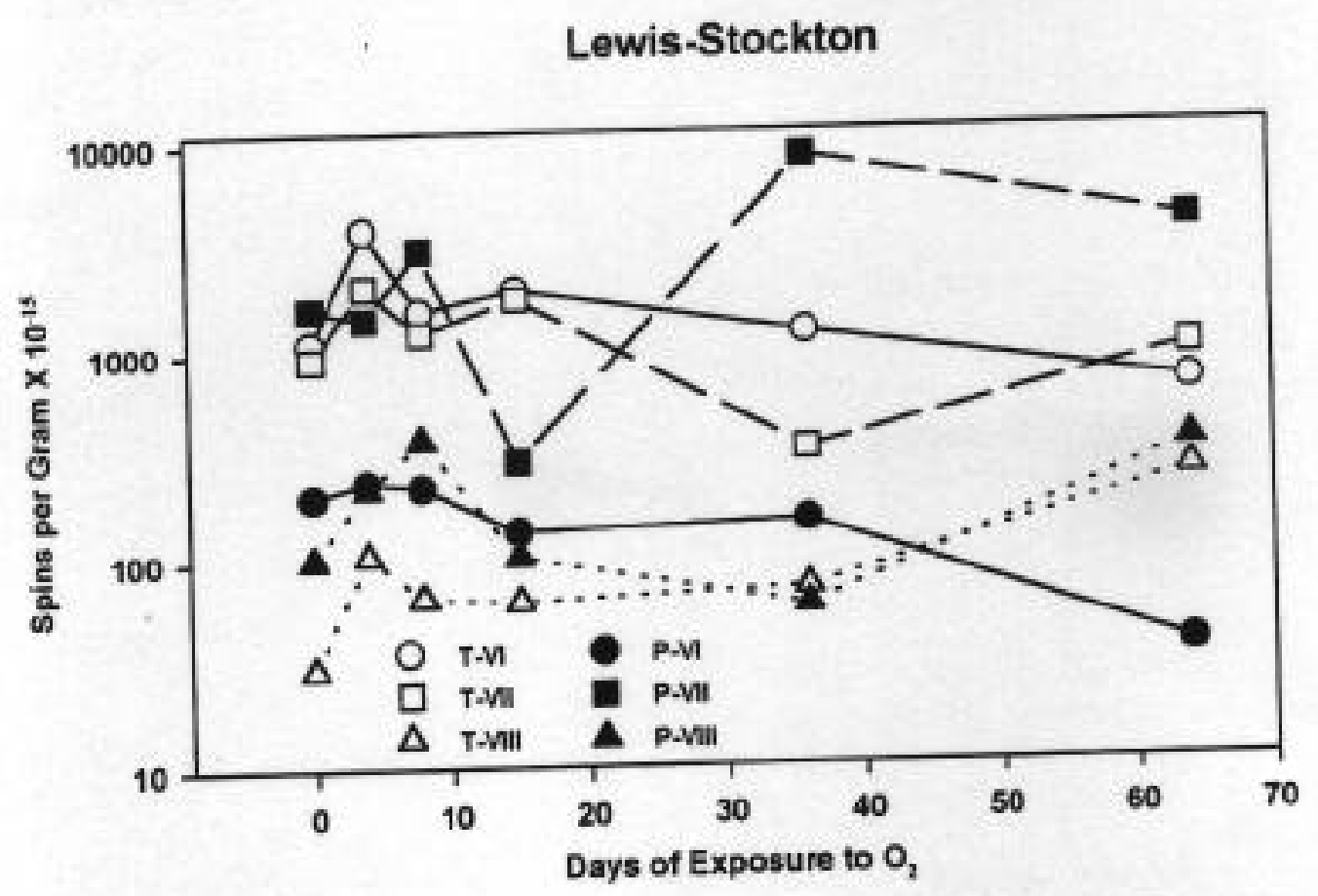

Figure 27. The retention of spin probes VI (O), VII $(\square)$ and VIII $(\Delta)$ in vacuum dried Lewiston-Stockton coal swelled in toluene (open symbols) and pyridine (closed symbols) as a function of oxidation period expressed in days.

Initially, vacuum dried Lewiston-Stockton coal exhibits a stronger concentration of $\mathrm{COOH}$ since the retention of spin probe VII concentration in pyridine swelled coal is much greater than that of spin probe VI. The same is not observed for toluene swelled coal, for which spin probes VI and VII exhibit similar retentions. In this case, the more easily accessible regions contain more $\mathrm{OH}$ groups or more equal distributions of $\mathrm{C}=\mathrm{O}$ and $\mathrm{COOH}$ groups. After 4 days of exposure to oxygen, the retention of spin probe VIII increases in both toluene and pyridine swelled coal. This indicates that the covalent structure of the coal has been disrupted and opened up enough to allow additional access of the spin probes. Since the increase observed for spin probe VIII in toluene swelled coal is greater than that for pyridine swelled coal, more accessible regions are created in the area limited by the hydrogen bond interactions. This is apparent by observing the retention of the polar spin probes swelled in toluene. Both are observed to increase in concentration, indicating more accessible regions and perhaps an increase in $\mathrm{OH}$ functional groups. The retention of the polar spin probes in pyridine swelled coal does not change very much after 4 days of exposure to oxygen. The slight increase in retention of spin probe VI along with the slight decrease in the retention of spin probe VII might indicate that some of the $\mathrm{OH}$ groups are being converted into $\mathrm{C}=\mathrm{O}$ functionalities. The retention of spin probes VI and VIII are equal in pyridine swelled coal. Since spin probe VIII has no functionality, it is likely that some of the new accessible regions posess non-polar surfaces.

At 8 days of exposure, the retention of all three spin probes in toluene swelled Lewiston-Stockton coal has decreased. This indicates an increase in hydrogen bond interaction which limits the accessibility in toluene swelled coal. The retention of spin probe VIII in pyridine swelled coal continues to increase to a concentration significantly greater than that observed for spin probe VI. The increase in spin probe VIII retention 
indicates further degradation of covalent cross-linking and increased access to the micropore structure. The retention of spin probe VII in pyridine swelled coal also increased while the concentration of spin probe VI remains unchanged. The greater observed retention of spin probe VIII over VI in pyridine swelled coal provides additional evidence that the breaking of covalent cross-linking initially exposes micropore areas which are non-polar in nature. The increase in retention of spin probe VII shows that some of the previously formed $\mathrm{C}=\mathrm{O}$ groups are being further oxidized to $\mathrm{COOH}$.

As the exposure period is increased to 15 days, the retention of spin probe VIII in toluene swelled coal remains unchanged. There is therefore little change in the physical structure of the coal limited by hydrogen bond interactions. A decrease is observed however, for the concentration of spin probe VIII in pyridine swelled coal. A decrease in concentration is also observed for spin probes VI and VII in pyridine swelled coal. Since all three spin probes exhibit a decrease in retention in the pyridine swelled coal, it is likely that peroxide radicals formed upon oxidation of the most recently formed non-polar areas are terminating in covalent cross-links which limit the access of the spin probes in the coal structure. Both of the polar spin probe concentrations increase slightly in toluene swelled coal. Since the concentration of these polar spin probes in toluene swelled coal exceeds those of the polar spin probes in pyridine swelled coal, it is apparent that the hydrogen bonded structure is opened enough so that the spin probes have access to the smaller micropores. However, when the these hydrogen bond interactions are disrupted, as in the case where pyridine is used as a swelling solvent, even the polar spin probes are not retained. This indicates that some steric support is necessary for significant retention of guest molecules in coal structure.

At 36 days of exposure, the retention of spin probe VIII decreases in the case of pyridine swelled coal and increases slightly in the case of toluene swelled coal. The decrease observed for spin probe VIII in pyridine swelled coal indicates an increase in covalent cross-linking if similar results are seen in the retention character of the polar spin probes in pyridine swelled coal, or an increased opening of the structure to the point where spin probes are washed out if increases in the polar spin probes are observed. The slight increase in spin probe VIII retention in toluene swelled coal could be attributed to a slight change in the hydrogen bonded character of the coal. The retention of the polar spin probes decreases in toluene swelled Lewiston-Stockton coal. The decrease is stronger in the case of spin probe VII. Since the concentration of both spin probes decrease, it is likely that the hydrogen bond cross-linking has increased. Because a decrease in the concentration of spin probe VIII was not likewise decreased, some newer, non-polar micropores must have become available to offset the increase in hydrogen bond interactions which limited the accessibility of the polar spin probes. Since the decrease for spin probe VII was so much stronger than the decrease observed for spin probe VI, the COOH groups must be used to a greater extent in forming these hydrogen bond cross-links. The concentration of spin probe VI in pyridine swelled coal increases only slightly, while the concentration of spin probe VII increases by more than an order of magnitude. The increases observed are partially due to a further opening of the structure by disruption of covalent cross-links, supported by the observed decrease in spin probe VIII. It is also likely that more of the non-polar micropores have been oxidized to contain more polar functionalities. It is also apparent from the large increase in spin probe VII retention that large amounts of $\mathrm{COOH}$ groups are being formed from the oxidation of $\mathrm{C}=\mathrm{O}$ and $\mathrm{OH}$ groups.

As the exposure period is increased from 36 days to 64 days, the retention of spin probe VIII is increase in both toluene and pyridine swelled Lewiston-Stockton coal. The strong change in spin probe VIII retention in the cases of both toluene and pyridine swelled coal indicates a change in the covalent structure of the coal. It is apparent that much of the covalent cross-linking has been disrupted, exposing additional micropore structure. Since 
the retention of spin probe VIII in pyridine swelled coal exceeds the concentration of spin prove VI by about an order of magnitude, it is clear that the new micropore areas exposed are non-polar in nature. This is further supported by the decrease in retention observed for both of the polar spin probes in pyridine swelled coal. The decrease in spin probe VII retention might also be accounted for by oxidation of $\mathrm{COOH}$ groups to $\mathrm{CO}_{2}$, but in any case, it can be asserted that any additional formation of $\mathrm{COOH}$ at this point is offset by a structural opening which does not include a highly polar character. The strong decrease in the retention of spin probe VI in pyridine swelled coal indicates that the hydrogen bond acceptor groups such as $\mathrm{C}=\mathrm{O}$ have been converted to $\mathrm{COOH}$ or other hydrogen bond donors. Also, no further production of polar regions in the coal structure have been created.

\section{Pittsburgh \#8 Coal (85.5\% carbon dmmf)}

In Figure 28, the retention of spin probes VI, VII and VIII in vacuum dried Pittsburgh \#8 coal swelled in both pyridine and toluene is expressed as a function of oxidation period. The coals were all exposed to dry oxygen at $0 \%$ humidity. In the case of coal swelled in pyridine, the data points are marked by solid figures, and in the case of toluene, the data points are marked by open figures. Spin probe VI is represented by circles, spin probe VII by squares and spin probe VIII by triangles. The retention data was scaled logarithmically so that all of the spin probes could be plotted on the same graph. Also, some of the more significant changes are easier to see.

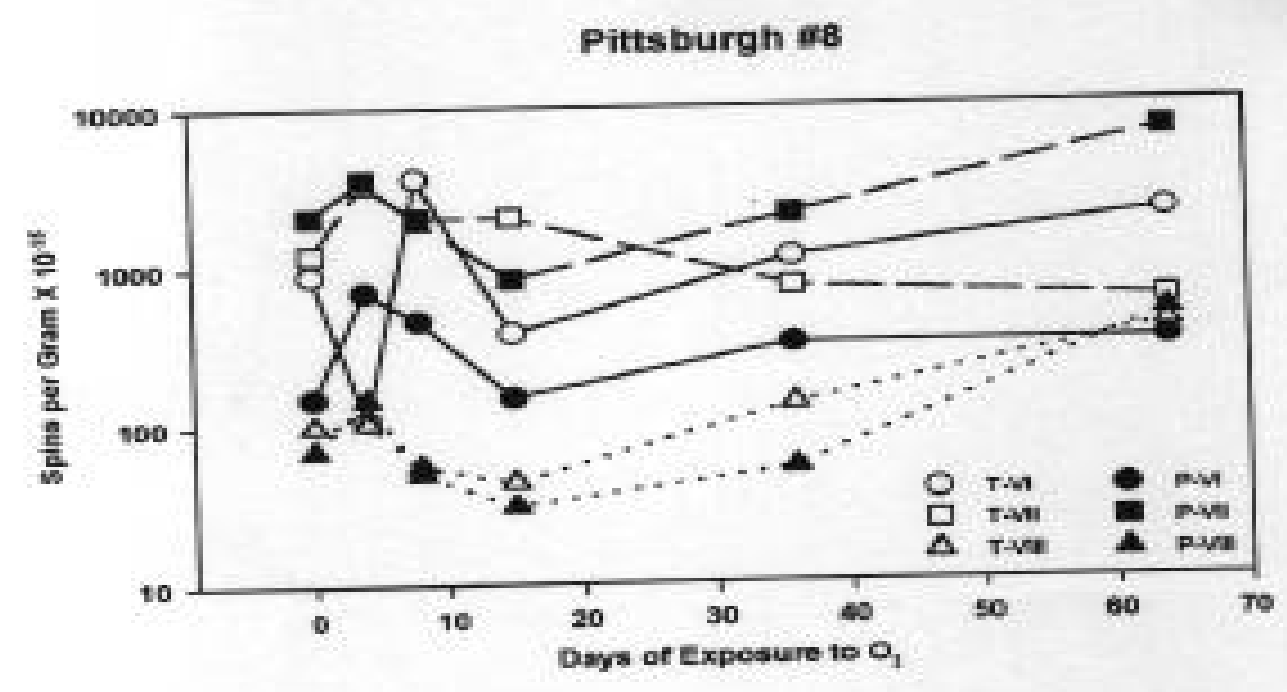

Figure 28. The retention of spin probes VI in vacuum dried Pittsburgh \#8 coal swelled in toluene as a function of oxidation period expressed in days.

Upon the initial exposure of 4 days to the oxygen environment, the retention of all of the spin probes in pyridine swelled Pittsburgh \#8 coal increase significantly. This indicats that the covalent cross-links in the coal structure were disrupted at this point, allowing increased access of guest molecules to the coal structure. The greater increase exhibited by spin probe VI suggests that in the opened structure there was a greater concentration of hydrogen bond acceptors like $\mathrm{C}=\mathrm{O}$ groups. The retention of spin probe VIII in toluene swelled coal remains unchanged by the initial exposure to oxygen. This suggests that there is very little overall change in the physical character of the hydrogen bonded structure. The change in retention characteristics of the polar spin probes in toluene swelled coal are drastically different. The retention of spin probe VII in toluene swelled coal increases significantly, while the concentration of spin probe VI in toluene swelled 
coal decreases by nearly an order of magnitude. In fact, the concentration of spin probe VI is reduced to a point where it is not retained any more than spin probe VIII. This indicates a complete reduction in the concentration of hydrogen bond acceptor groups in the micropore structure available in toluene swelled coal. It can therefore be suggested at this point that most of the $\mathrm{C}=\mathrm{O}$ has been converted to $\mathrm{COOH}$ or is used in forming the hydrogen bonded cross-linking in Pittsburgh \#8 coal. The increase in retention of spin probe VII in toluene swelled coal supports the formation of $\mathrm{COOH}$, especially since the amount of increase exceeds the reduction observed in the concentration of spin probe VI.

At 8 days of exposure of the vacuum dried coal to oxygen, a significant decrease in the retention of all three spin probes in pyridine swelled coal is observed. The similar decrease observed for these spin probes suggests that some of the covalent cross-links are being restored by radical termination reactions during the oxidation process. Not much change is observed in the relative concentration of the polar groups in the coal structure. The retention of spin probe VIII in toluene swelled coals likewise exhibits a decrease. This could be caused by the increase in covalent cross-linking or additional hydrogen bond cross-linking. The concentration of spin probe VII in toluene swelled coal exhibits a decrease similar to that observed for spin probe VIII. This was primarily caused by the increased structural limitations imposed on the accessibility brought about by increased cross-linking. It is also possible that some of the $\mathrm{COOH}$ is being further oxidized to $\mathrm{CO}_{2}$. The concentration of spin probe VI in toluene swelled coal shows the largest change, as it increases by more than an order of magnitude. At this point, much of the structure limited by hydrogen bonding is affected by changes which bring about a large increase in the $\mathrm{C}=\mathrm{O}$ group concentration. Since at this point, the spin probe VI retention is much higher than any of the others, it is likely that the hydrogen bonded cross-linking brought about structural constraints which provide steric assistance in trapping probe molecules in toluene swelled coal.

As the oxidation period is increased to 15 days, a continued decrease is observed for the retention of all three spin probes in pyridine swelled coal. This provides indication that a continued increase in covalent cross-linking has occurred. Because the changes in pyridine swelled coal have been very similar up to this point, it is likely that most of the changes exhibited by covalent structure of Pittsburgh \#8 coal are due to changes in the cross-linking. The relative distribution of polar sites seems to remain fairly constant. The concentration of spin probe VIII in toluene swelled coal exhibits a slight decrease, indicating an increase in hydrogen bonded cross-linking. The concentration of spin probe VI in toluene swelled coal is again greatly reduced. This would once again show that the $\mathrm{C}=\mathrm{O}$ groups are oxidized to $\mathrm{COOH}$. Because the retention of spin probe VII does not change, it is likely that the formation of $\mathrm{COOH}$ is offset by the increased hydrogen bond interactions which inhibit intercalation of guest molecules.

After the Pittsburgh \#8 coal has been oxidized for 36 days, all three spin probes exhibit an increase in retention in pyridine swelled coal. This suggests that the previously formed covalent cross-links have started to degrade. The peroxide cross-links are most likely being oxidized to $\mathrm{OH}$ groups, although the stronger retention of spin probe VII suggests that the concentration of $\mathrm{COOH}$ in the coal structure at this point is slightly higher. The retention of spin probe VIII in toluene swelled coals increased significantly, indicating disruption of hydrogen bond cross-linking. The concentration of spin probe VI in toluene swelled coal increased similarly. The reduction of spin probe VII retention in toluene swelled coal suggests that the concentration of $\mathrm{COOH}$ was reduced. This could only be accounted for by oxidation of $\mathrm{COOH}$ to $\mathrm{CO}_{2}$. 
Finally, when the oxidation period is increased from 36 to 64 days, the retention of spin probe VIII in pyridine swelled coal increases nearly an order of magnitude to a concentration greater than that observed for spin probe VI in pyridine swelled coal. This indicates that the covalent cross-linking was further degraded during the oxidation process, opening up more of the micropore structure, and that much of this structure is non-polar in character. The retention of spin probe VIII in toluene swelled coal also increased. This may be partially due to the decreased covalent cross-linking, but some disruption of the hydrogen bonded cross-linking must occur to allow significant access since extensive hydrogen bond interactions occur in coal structures which have less covalent cross-linking. The retention of spin probe VI in pyridine swelled coal remains unchanged while the retention of spin probe VII in pyridine swelled coal increases significantly. This suggests that some of the other functionalities are being converted to $\mathrm{COOH}$. The retention of spin probe VI in toluene swelled coal continued to increase while the concentration of spin probe VII in toluene swelled coal decreased slightly. This demonstrates that the chemical changes in the more accessible regions are much different than those observed by the structure opened by pyridine. It would appear that some of the $\mathrm{COOH}$ in this region is further oxidized to $\mathrm{CO}_{2}$, while additional $\mathrm{OH}$ groups are converted to $\mathrm{C}=\mathrm{O}$ groups.

\section{Upper Freeport Coal (88.1\% carbon dmmf)}

In Figure 29, the retention of spin probes VI, VII and VIII in vacuum dried Upper Freeport coal swelled in both pyridine and toluene is expressed as a function of oxidation period. The coals were all exposed to dry oxygen at $0 \%$ humidity. In the case of coal swelled in pyridine, the data points are marked by solid figures, and in the case of toluene, the data points are marked by open figures. Spin probe VI is represented by circles, spin probe VII by squares and spin probe VIII by triangles. The retention data was scaled logarithmically so that all of the spin probes could be plotted on the same graph. Also, some of the more significant changes are easier to see.

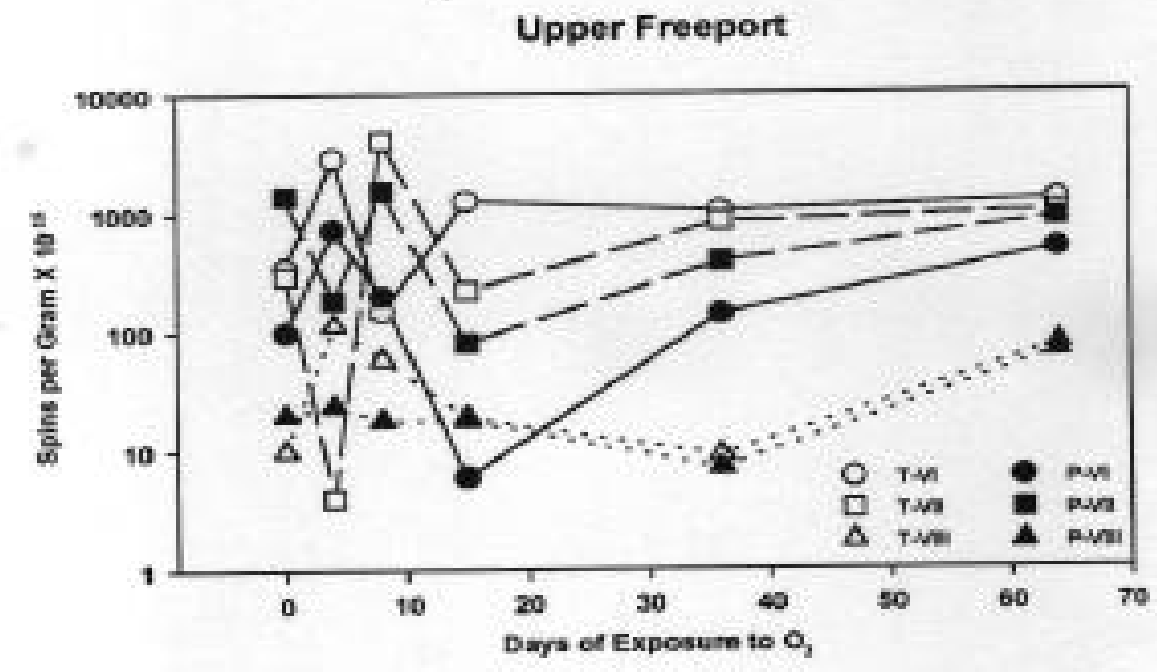

Figure 29. The retention of spin probes VI and VIII in vacuum dried Upper Freeport coal swelled in toluene (open symbols) and pyridine (closed symbols) as a function of oxidation period expressed in days.

After the initial exposure of Upper Freeport coal to oxygen for 4 days, the concentration of spin probe VIII in pyridine swelled coal did not change significantly, and 
the concentration of spin probe VIII in toluene increased by an order of magnitude. It seems that the covalent cross-linking of the coal structure is not significantly altered in the first four days. However, the hydrogen bonded structure is significantly altered. The increased retention of spin probe VIII in toluene swelled coal indicates that the hydrogen bonded cross-linking was disrupted to a large extent. The retention of spin probe VI is observed to increase in the case of both toluene and pyridine swelled coal. It is apparent that $\mathrm{C}=\mathrm{O}$ groups are being formed at this point, especially for the pyridine swelled coal, since the covalent structure was not opened to any extent. The concentration of spin probe VII, on the other hand, was observed to decrease in both toluene and pyridine swelled coal. The decrease in retention was strongest in the case of toluene swelled coal, in which the spin probe VII concentration dropped two orders of magnitude. It seems that there is very little $\mathrm{COOH}$, and that the $\mathrm{OH}$ groups were converted almost exclusively to $\mathrm{C}=\mathrm{O}$. In the case of toluene swelled coal, the concentration of spin probe VII drops far below that of spin probe VIII, indicating that in the more easily accessible areas of the micropore structure, some of the micropore character must be non-polar to a significant extent.

After 8 days of exposure to oxygen, the retention of spin probe VIII in pyridine swelled coal does not change significantly. This is an indication that the covalent crosslinks in Upper Freeport coal are not significantly disrupted. The retention of spin probe VIII in pyridine swelled coal, however, does decrease. This provides evidence that the hydrogen bonded cross-linking is increased so that the access of guest molecules in toluene swelled coal is inhibited. The retention of spin probe VI in both toluene and pyridine swelled coal is greatly reduced. This reduction is far greater in the case of toluene swelled coal. This indicates that the $\mathrm{C}=\mathrm{O}$ groups are being converted to $\mathrm{COOH}$. This trend is more strongly observed in the case of toluene because of the additional hydrogen bonded structure. The retention of spin probe VII increases sharply for the case of both toluene and pyridine swelled coal. This increase is equal to three orders of magnitude in the case of toluene swelled coal, and is a strong indication that large amounts of $\mathrm{COOH}$ are being produced at this stage in the oxidation process. These changes are more significant in the micropore areas not limited by the hydrogen bond interactions.

As the oxidation period is increased to 15 days, the retention of spin probe VIII in pyridine swelled coal still shows no significant change, indicating that the covalent crosslinks have still not been altered to any significant extent. The retention of spin probe VIII in toluene swelled coal exhibits a significant decrease, suggesting a further increase in hydrogen bond cross-linking. The retention of spin probe VII exhibits a strong decrease in both toluene and pyridine swelled coal. Some of the decrease observed for the case of toluene swelled coal could be due to the increased hydrogen bonding. The strong decrease observed for spin probe VII could only be explained by a structural inhibition or a strong decrease in the concentration of hydrogen bond donor groups such as $\mathrm{COOH}$ and $\mathrm{OH}$. Since there was no observed decrease in the concentration of spin probe VIII in the case of pyridine swelled coal at least, structural inhibition is not likely. Therefore, a process where $\mathrm{COOH}$ is removed by further oxidation to $\mathrm{CO}_{2}$ is much more plausible. The retention of spin probe VI in pyridine swelled coal is decreased by well over an order of magnitude. The concentration of spin probe VI in pyridine swelled coal is actually less than the concentration observed for spin probe VIII under the same circumstances. This indicates that the structure available during pyridine swelling has a significant non-polar character and further supports the removal of oxygenated species by oxidation to $\mathrm{CO}_{2}$. The concentration of spin probe VI in toluene swelled coal is greatly increased at this point. Since the decrease observed for spin probe VII was so strong, it is likely that some of the $\mathrm{OH}$ concentration in this easily accessible region is being oxidized to $\mathrm{C}=\mathrm{O}$. 
After 36 days of exposure of the vacuum dried Upper Freeport coal to the oxygen environment, a decrease in retention is observed for spin probe VIII in both toluene and pyridine swelled coal. This is indication that the covalent cross-linking in the coal structure had been disrupted to a point where spin probes could be washed out since there is no observed decrease in the concentration of the polar spin probes. It is also possible that the number of non-polar micropores is reduced at this point. The retention of both of the polar spin probes in pyridine swelled coal is increased. This increase is much stronger for spin probe VI than for VII. This suggests the non-polar regions are being oxidized to mostly $\mathrm{OH}$ and $\mathrm{C}=\mathrm{O}$. Only a slight decrease is observed in the retention of spin probe VI in toluene swelled coal. The retention of spin probe VII in toluene swelled coal is significant, indicating that some of the $\mathrm{C}=\mathrm{O}$ groups are being converted to $\mathrm{CO}_{2}$ in the micropore region accessible during toluene swelling.

As the oxidation period is increased to 64 days, a large increase in retention is observed for spin probe VIII in both toluene and pyridine swelled coal. This suggests that further degradation of the covalent cross-linking occurred to expose more of the micropore structure. The retention of both polar spin probes is increased in pyridine swelled coal. This is most likely due to the increase in the microporous area now available to guest molecules. Since the concentration of spin probe VII is still significantly greater that spin probe VI, it is likely that there is still a higher concentration of $\mathrm{COOH}$ groups in the structure available during pyridine swelling. As no significant change is observed in the retention of the polar spin probes in toluene swelled coal, it is likely that some of the new micropore areas made available in the structure limited by hydrogen bond interactions are non-polar in character.

\section{Poccahontas \#3 Coal $(91.8 \%$ carbon dmmf)}

In Figure 30, the retention of spin probes VI, VII and VIII in vacuum dried Poccahontas \#3 coal swelled in both pyridine and toluene is expressed as a function of oxidation period. The coals were all exposed to dry oxygen at $0 \%$ humidity. In the case of coal swelled in pyridine, the data points are marked by solid figures, and in the case of toluene, the data points are marked by open figures. Spin probe VI is represented by circles, spin probe VII by squares and spin probe VIII by triangles. The retention data was scaled logarithmically so that all of the spin probes could be plotted on the same graph. Also, some of the more significant changes are easier to see.

After the initial oxidation at four days of exposure, the retention of all three spin probes in toluene or pyridine swelled coal increases significantly. The retention of spin probe VIII in both pyridine and toluene swelled coal exhibits an increase of one order of magnitude. The similar behavior of the spin probe VIII retention in toluene and pyridine swelled coal is due primarily to changes in the covalent structure. The large increase in spin probe VIII retention in pyridine swelled coal indicates that a significant amount of the covalent cross-linking in the coal was disrupted to expose much of the micropore network. This increase in the number of micropores also accounts for the increase in retention observed for the polar spin probes. 


\section{Poccahontas \#3}

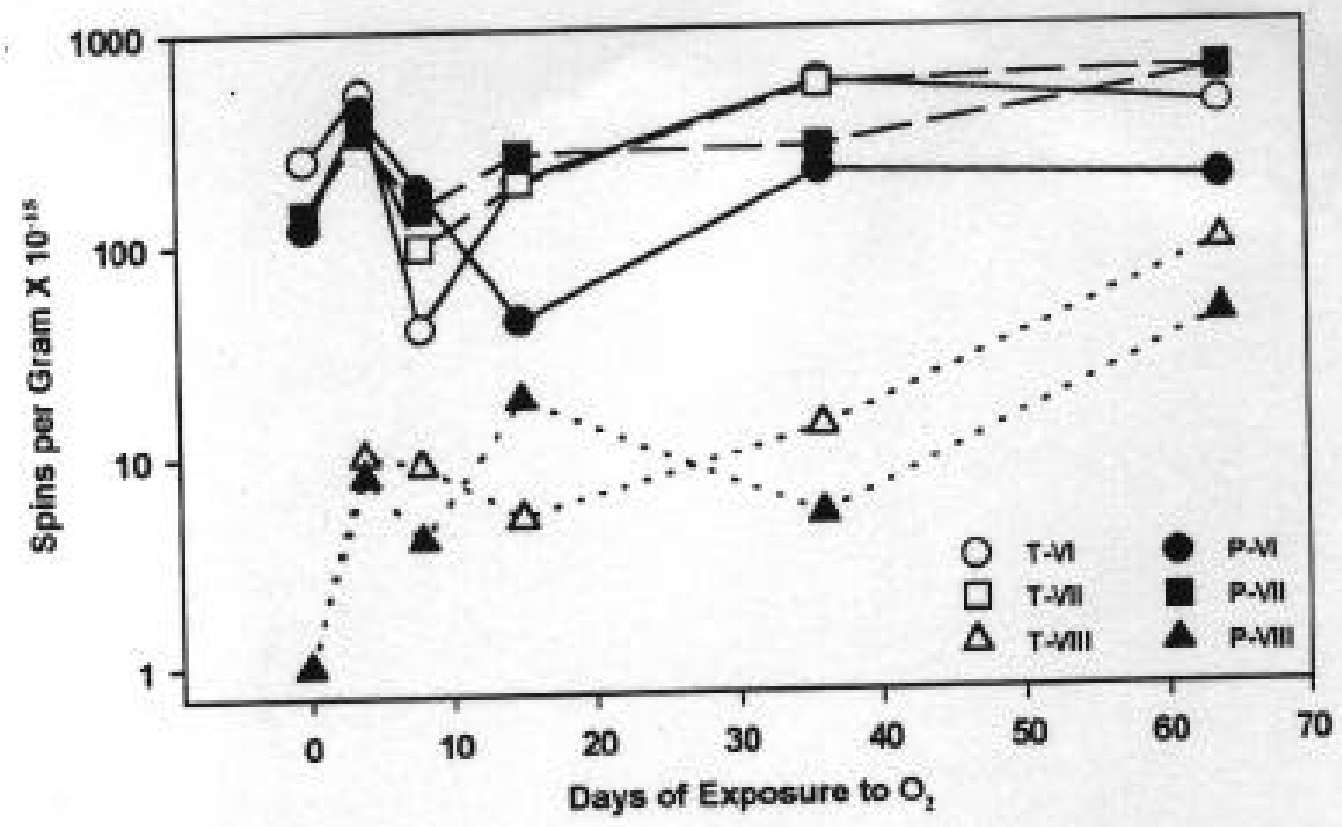

Figure 30. The retention of spin probes VI in vacuum dried Poccahontas \#3 coal swelled in toluene (open symbols) and pyridine (closed symbols) as a function of oxidation period expressed in days.

At 8 days of exposure to oxygen, a decrease in retention is observed for all of the spin probes in both toluene and pyridine swelled Poccahontas \#3 coal. The similar decrease in the retention of all three spin probe in pyridine swelled coal suggests that the covalent cross-linking increased. The retention of spin probe VIII in toluene swelled coal did not really change very much. This indicates that, even though the covalent crosslinking increased, the hydrogen bonded structure did not changed as much. Therefore, the decrease in retention observed for the polar spin probes in toluene swelled coal can only be partially due to increase hydrogen bonding, and suggests a reduction of polar functionalities. The greater decrease observed for the retention of spin probe VI as opposed to spin probe VII indicates that a higher concentration of $\mathrm{COOH}$ exists at this point.

After 15 days of oxidation, the retention of spin probe VIII in pyridine swelled coal is strongly increased, indicating further disruption of the covalently cross-linked structure so that more of the micropore structure can be revealed. The retention of spin probe VIII in toluene swelled coal, on the other hand, is shown to decrease, indicating an increase in hydrogen bond interactions and cross-linking. The retention of both polar spin probes in toluene swelled coal is observed to increase significantly. This indicates that the concentration of $\mathrm{OH}$ groups is greatly increased, especially since the hydrogen bond crosslinking was increased. The concentration of spin probe VII in pyridine swelled coal increased, indicating increased concentration of $\mathrm{COOH}$ groups. The concentration of spin probe VI in pyridine swelled coal decreased, suggesting that the increase in $\mathrm{COOH}$ groups was brought about by oxidation of the $\mathrm{C}=\mathrm{O}$ concentration, resulting in a decrease in hydrogen bond acceptors. 
When the Poccahontas \#3 coal has been exposed to oxygen for 36 days, a strong decrease in the retention of spin probe VIII in pyridine swelled coal is observed. Since none of the other spin probes exhibit a decrease in retention at this point, it is more probable that the covalent cross-linking is further disrupted to a point where the small, nonpolar spin probe is easily washed out. It is also likely that some of the non-polar regions have been oxidized and contain polar functionalities. The retention of all three spin probes in toluene swelled coal is observed to increase significantly, suggesting that the hydrogen bonded cross-linking in the coal structure prevents the structure from opening to such an extent that the smaller spin probes are washed out, even though the covalent structure was opened further. The retention of spin probe VII remains unchanged in pyridine swelled coal while the concentration of spin probe VI is increased, indicating that $\mathrm{OH}$ is being oxidized to $\mathrm{C}=\mathrm{O}$ while the concentration of $\mathrm{COOH}$ remains fairly constant.

As the oxidation period is increased to 64 days, an increase in the retention of spin probe VIII is observed for both toluene and pyridine swelled coal. This points to changes in the covalent structure since the spin probe concentration increases similarly in both cases. The increase of spin probe VIII observed in pyridine swelled coal indicates continued degradation of the covalent cross-linking in the coal structure, providing additional access to the microporous regions. A slight decrease in the retention of spin probe VI in both toluene and pyridine swelled coal indicates that the $\mathrm{C}=\mathrm{O}$ groups are being oxidized to $\mathrm{COOH}$. This is verified by the increased retention observed for spin probe VII in both pyridine and toluene swelled coal, although the increase observed in the case of toluene swelled coal is very slight.

\section{Summary of the Oxidation Process in Vacuum Dried Coals}

The general retention behavior of each spin probe/solvent combination during the oxidation process has been previously discussed in terms of coal rank by the use of three dimensional plots. To obtain more detailed insight into the oxidation process, the retention data was plotted two dimensionally for each individual coal. The spin concentration was expressed logarithmically so that the data for each spin probe/solvent combination could be plotted on the same graph. By close examination and comparison of the retention behavior of each spin probe in both toluene and pyridine swelled coal, a more complete understanding of the oxidation process in vacuum dried coals can be gained.

The process observed during the oxidation period for the APCS coals goes as follows: $\mathrm{R}^{+}+\mathrm{O}_{2}$ to $\mathrm{ROO}^{+}$, $\mathrm{ROOH}$ or ROOR, $\mathrm{ROOH}$ to $\mathrm{ROH}$ or $\mathrm{OROH}, \mathrm{ROH}$ to $\mathrm{R}=\mathrm{O}$, $\mathrm{R}=\mathrm{O}$ to $\mathrm{R}-\mathrm{COOH}$.

Methods for determining structural changes by observing changes in spin probe retention are summarized below. It is important to remember that the changes in spin probe retention observed in coals swelled in pyridine are reflective of oxidational changes in the covalent structure of the coal since pyidine is known to disrupt the hydrogen bonded structure. The changes observed in the retention of spin probes in toluene swelled coals are reflective of oxidational changes in both the covalent and hydrogen bonded structure of the coal. Therefore, the use of toluene as a swelling solvent may provide a more general picture of the inclusion process in coal. However, the use of pyridine allows for examination of the opened coal structure, and when compared to results of the use of toluene, provides a complete picture of the inclusion of spin probes in coal. 
Spin Probe VIII

Pyridine $+\quad$ Increase indicates opening of the covalent structure to expose more of the microporous structure by disruption of covalent cross-linking. Decrease indicates increased cross-linking of the covalent structure to inhibit the accessibility of guest molecules, or an opening of the covalent structure to such an extent that the microporous structure is disrupted and the spin probe are being washed out.

* If the concentration of spin probe VIII is higher than either of the polar spin probes, there is indication that the exposed microporous region is non-polar in character.

Toluene $\quad+\quad$ Increase indicates an opening of the covalent or hydrogen bonded structure to expose more of the microporous regions by disruption of covalent or hydrogen bonded cross-linking.

- Decrease indicates increased cross-linking of the covalent or hydrogen bonded structure to inhibit the intercalation of guest molecules, or an opening of the hydrogen bonded structure to such an extent that the spin probes are washed out.

* It is possible to see a decrease in covalent cross-linking and an increase in hydrogen bond cross-linking since the disruption of covalent cross-links can result in the formation of polar substituents which exhibit hydrogen bond interactions. This is indicated when the changes in retention of spin probe VIII are varied between pyridine and toluene swelled coal.

* If the changes in retention of spin probe VIII in toluene swelled coal closely follow the changes observed for spin probe VIII in pyridine swelled coal, then retention variations are largely due to changes in the covalent structure.

Spin Probe VI Pyridine $\quad+$

Toluene $\quad+\quad$ An increase indicates disruption of hydrogen bond interaction which exposes more of the micropore structure, or a decrease in covalent cross-linking, but a slight increase in hydrogen bonded cross- 
linking which provides some steric support of the intercalation of guest molecules, or an increase in the concentration of $\mathrm{OH}$ or $\mathrm{C}=\mathrm{O}$ groups in the region limited by hydrogen bond interactions.

- A decrease indicates increased covalent or hydrogen bond crosslinking which limits the access of guest molecules to the structure, or a reduction in the $\mathrm{OH}$ or $\mathrm{C}=\mathrm{O}$ (hydrogen bond acceptor) concentration in the region limited by the hydrogen bond interactions, or a reduction of the polar substituent in the micropore region limited by the hydrogen bond interactions.

* If a strong difference is observed in the retention changes of spin probe VI between pyridine and toluene swelled coal, the functionality of the polar substituents most involved in the hydrogen bond interactions can be discerned.

\section{Spin Probe VII

Pyridine $\quad+$

$+\quad$ An increase indicates an opening of the covalent structure to explore more of the microporous region, or an increase in the formation of $\mathrm{COOH}$ (hydrogen bond donor) groups, or an increase in groups which behave as both hydrogen bond acceptors and donors, like $\mathrm{OH}$ groups.

- A decrease indicates increased covalent cross-linking which results in the reduction in accessibility of guest molecules to the structure, or a reduction in $\mathrm{OH}$ or $\mathrm{COOH}$ (hydrogen bond donor) concentration, or a reduction in the concentration of polar groups in the micropore structure.

* $\quad$ Spin probe VII often works in conjunction with spin probe VI. If the retention behavior differs, it is likely that chemical changes are taking place. If the retention behavior is very similar, changes in physical structure or overall polar functionality are indicated.

* Where the concentration of $\mathrm{COOH}$ is observed, it is likely that the carboxylic acid is oxidized to $\mathrm{CO}_{2}$.

Toluene $\quad+\quad$ An increase indicates disruption of hydrogen bond interaction which exposes more of the micropore structure, or a decrease in covalent cross-linking, but a slight increase in hydrogen bonded crosslinking which provides some steric support of the intercalation of guest molecules, or an increase in the concentration of $\mathrm{OH}$ or $\mathrm{COOH}$ groups in the region limited by hydrogen bond interactions.

A decrease indicates increased covalent or hydrogen bond crosslinking which would limit the access of guest molecules to the structure, or a reduction in the $\mathrm{OH}$ or $\mathrm{COOH}$ (hydrogen bond acceptor) concentration in the region limited by the hydrogen bond interactions, or a reduction of the polar substituent in the micropore region limited by the hydrogen bond interactions.

* If a strong difference is observed in the retention changes of spin probe VII between pyridine and toluene swelled coal, the 
functionality of the polar substituents most involved in the hydrogen bond interactions can be discerned.

* Oxidational changes which take place at different rates in the more accessible regions limited by hydrogen bond interactions and the rest of the micropore region limited only by covalent cross-linking can be observed by comparing results obtained for the polar spin probes in pyridine swelled coal versus those obtained for toluene swelled coal.

1. Hydroperoxides and some $\mathrm{C}-\mathrm{OH}$ are formed resulting in increased hydrogen bonding.

2. Condensation of peroxide radicals and relocated radicals results in increased covalent cross-linking in the coal structure.

3. Covalent cross-links are degraded and formation of $\mathrm{C}-\mathrm{OH}$ continues.

4. $\mathrm{C}-\mathrm{OH}$ is converted to $\mathrm{C}=\mathrm{O}$.

5. As more cross-linking is disrupted, more small micropore areas are exposed.

6. $\mathrm{C}=\mathrm{O}$ is converted to $\mathrm{COOH}$.

7. $\mathrm{COOH}$ is converted to $\mathrm{CO}_{2}$ and more non-polar micropore areas are exposed.

\section{SHORT TERM OXIDATION}

\section{Introduction}

Exposure of coal to air before the conversion of coal to liquid fuels of other chemicals has been a big concern in coal chemistry. ${ }^{59}$ It has been suggested that the structure of coal can be altered in as little as two minutes exposure to air. ${ }^{60}$ If so, this would make it difficult to carry out accurate structural studies on coal. The previous chapters have shown that both dehydration and oxidation change the physical and chemical structure in coal enough so that the molecular accessibility in coal is significantly altered. It is the goal of this research to determine how quickly and to what extent oxidation or dehydration can affect the structure of coal using the EPR spin probe technique. From the previous chapter on $\mathrm{O}_{2}$ oxidation, it was determined that Illinois \#6 coal exhibits the greatest changes in structure upon dehydration and oxidation. Lower ranked coals are more affected by water removal, but do not show as much change during oxidation. A flow of dry oxygen over the coal results in both loss of water and oxidation. A flow of argon, however, results in only dehydration since it is inert. By comparing the difference in the effects of both gases on coal, the effects of oxidation alone can be determined. Argon was chosen as the inert gas because of its ability to cover the samples completely with far less difficulty than nitrogen or other inert gases commonly used. Toluene was chosen as a swelling solvent because it does not significantly open the structure of the coal yet allows for diffusion of the spin probes into the available structure. Pyridine was used because it completely opens up the structure and allows for examination of hydrogen bonding sites in coal. Spin probe VIII is a small spherical shaped molecule with no polar substituents. The intercalation of this spin probe simply measures physical changes in the coal structure. Spin probe VI has a carboxyl group and is a hydrogen bond donor with the 
ability to detect polar interaction in the coal. Spin probe VII has an amino group and is a hydrogen bond acceptor with a much stronger ability to detect polar interactions than spin probe VI. By comparing the retention data for all three spin probes, a more complete picture of the nature of the probe-coal interactions can be obtained.

\section{Results in Toluene and Pyridine}

The effects of exposure of Illinois \#6 swelled in toluene to both argon and oxygen of the retention of spin probe VI are shown in Figure 31. Dramatic effects are seen on the retention of spin probe VI after just 30 seconds. After 30 seconds, a decrease of over 1200 $\mathrm{x} 10^{15}$ spins per gram is observed for coal exposed to argon. The decrease in retention is somewhat less pronounced for exposure to oxygen. After 5 minutes of exposure to argon, the retention characteristic of Illinois \#6 returned to that found for fresh coal. The increase for oxygen followed the same trend, but to a far less extent. At 5 minutes, the difference between dehydrated (argon only) and oxidized coal is significant. This difference becomes more pronounced after 50 minutes of exposure. In the first 5 minutes, oxidation seems to cause changes which counteract the effects of dehydration. Beyond 30 seconds, oxidation caused a decrease in the retention of spin probe VI, indicated by the increasing retention difference with exposure to oxygen as compared with argon. At 5 minutes or more, dehydrated coals (argon) have a much higher retention of spin probe VI than dehydrated and oxidized coal.

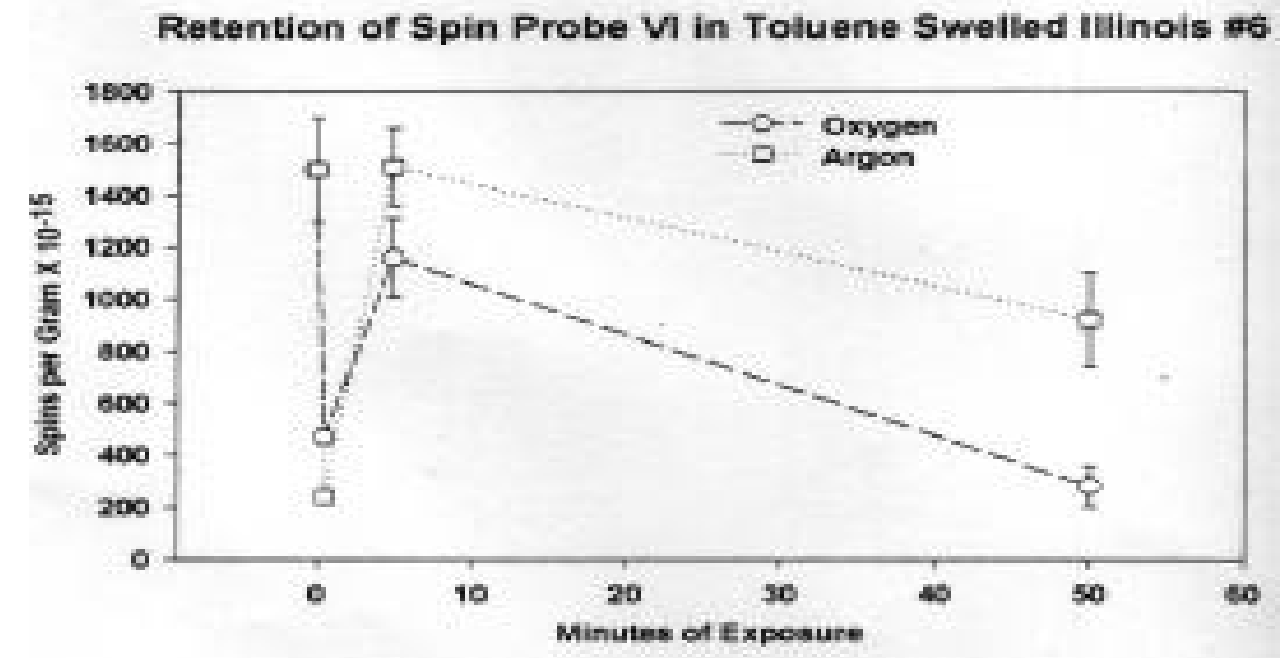

Figure 31. The retention of spin probe VI in toluene swelled Illinois \#6 coal exposed to both argon argon ( $(\square)$ and oxygen $(\mathrm{O})$.

The effects of dehydration and oxidation on the retention of spin probe VII in Illinois \#6 coal swelled in toluene are shown in Figure 32. A decrease in spin probe retention of $2500 \times 10^{15}$ spins per gram is observed upon exposure to either argon or oxygen for 30 seconds. This change represents an order of magnitude decrease in spin probe VII retention. This is followed by an increase in retention at 5 minutes of exposure, and then a more gradual decline in molecular accessibility for exposure up to 50 minutes. These results are similar to those obtained for spin probe VI retention over coal exposed to argon. This difference becomes less significant after 50 minutes of weathering. Again, the shape of each plot is very similar, indicating that the more severe changes are caused by dehydration rather than oxidation. 
Retention of Spin Probe VII in Toluene Swelled llinoins \#6

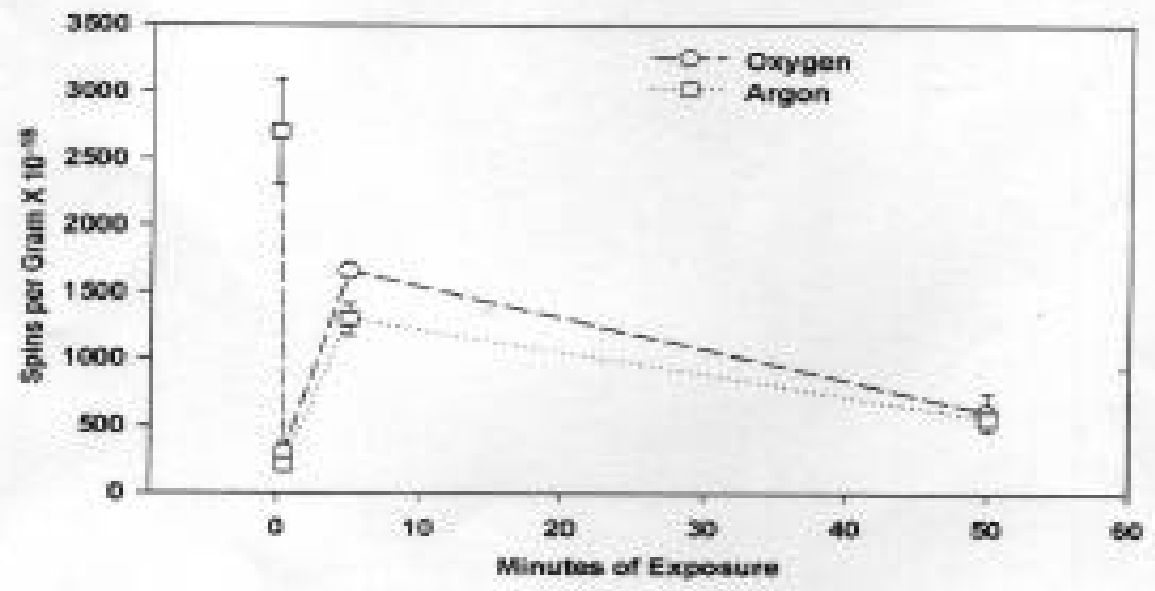

Figure 32. The retention of spin probe VII in toluene swelled Illinois \#6 coal exposed to both argon argon $(\square)$ and oxygen $(\mathrm{O})$.

Structural changes in Illinois \#6 coal swelled in toluene can be observed in Figure 33 by the retention of spin probe VIII. The plots generated are very similar to those shown in Figure 31 for spin probe VI. A decrease in retention is observed in the first 30 seconds, followed by an increase at five minutes and a gradual decrease in retention up to 50 minutes. The increase in spin probe retention exhibited at 5 minutes of exposure is much greater for coal exposed to argon than oxygen. The initial decrease in spin probe concentration is most likely due to a structural collapse or increased cross-linking, making the structure less accessible since the structure was likewise inaccessible to spin probes VI and VII. It also appears that the changes which occur due to oxidation counteract (to a limited degree) the changes brought about by dehydration in the first 5 minutes. Figure 33 shows that some of the effects seen in Figures 31 and 32 are due to changes in the physical structure since spin probe VIII has no reactive substituents, and shows similarly shaped plots. It is important to note that a retention of nearly $5.0 \times 10^{17}$ spins per gram is observed after 30 seconds of exposure to argon. The spin probe VIII concentrations found in Illinois \#6 coal fresh, vacuum dried or weathered in air were never observed to exceed $2.0 \times 10^{17}$ spins per gram.

Retention of Spin Probe VII in Toluene Swelled Illinois \#6

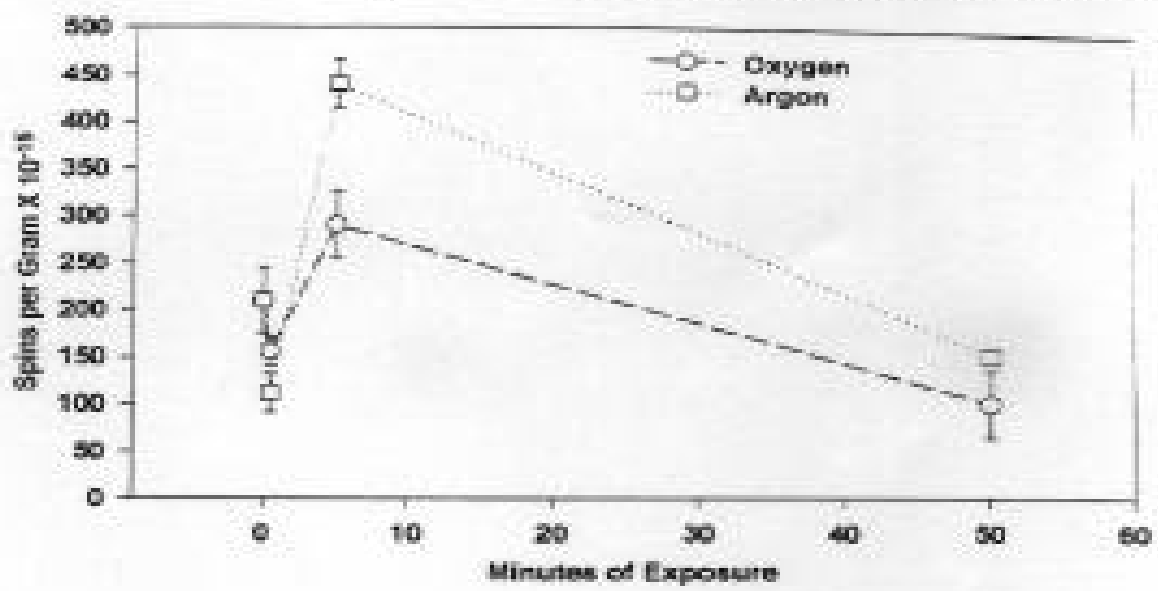

Figure 33. The retention of spin probe VIII in toluene swelled Illinois \#6 coal exposed to both argon argon $(\square)$ and oxygen $(\mathrm{O})$. 
When Illinois 6 is exposed to oxygen and argon and then swelled in pyridine in the presence of spin probe VI, retention characteristics exhibit changes illustrated in Figure 34 which are similar to those shown for coal swelled in toluene. A decrease in spin probe retention is observed after 30 seconds of exposure to oxygen or argon. Although this decrease is quite small in comparison to those shown for polar spin probes in toluene, it does indicate that the inaccessibility of these spin probes at 30 seconds of exposure is not entirely due to changes in physical structure, since pyridine is capable of disrupting most polar interactions in coal. Again, after 5 minutes of exposure, the retention of spin probe VI is increased to a point which is considerably greater than the initial retention, more so for coal exposed to oxygen than argon. As the time of exposure is extended to 50 minutes, the retention of spin probe VI in dried (exposed to argon) coal increased slightly while the retention in oxidized (exposed to oxygen) coal decreased.

Retention of Spin Probe V1 in Pyridine Swelled Illinois \#6

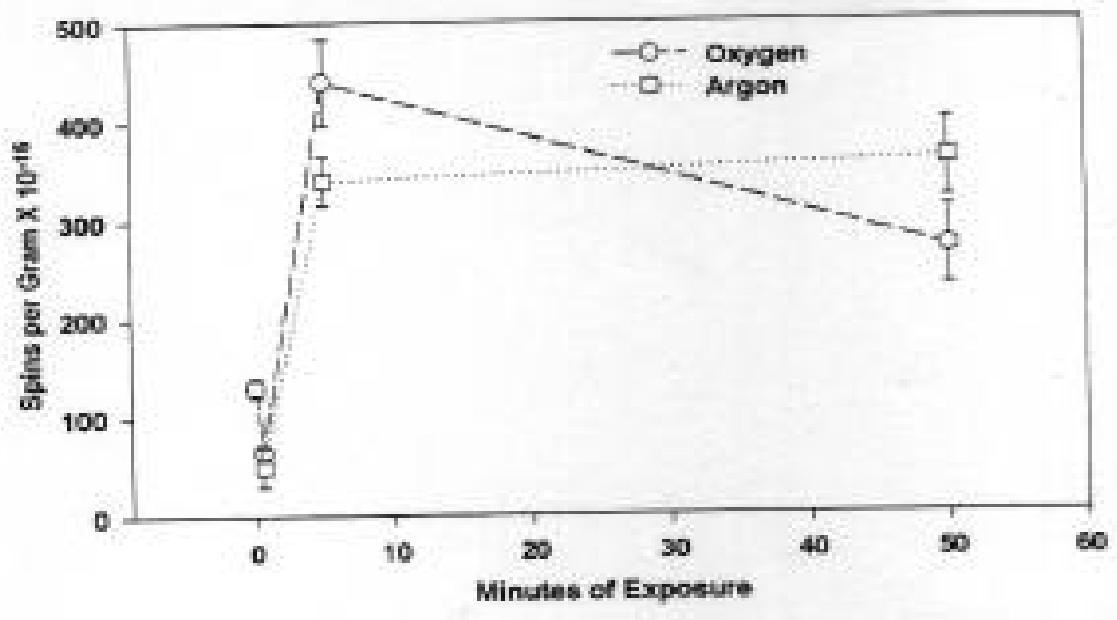

Figure 34. The retention of spin probe VI in pyridine swelled Illinois \#6 coal exposed to both argon $(\square)$ and oxygen $(\mathrm{O})$.

The results of changes in the retention of spin probe VII in Illinois \#6 coal upon exposure to oxygen and argon are shown in Figure 35. As seen in the previous figures, a decrease in retention is observed for exposure in both oxygen and argon, but more so for oxygen. After 5 minutes in argon, the coal exhibits improved retention, far surpassing the accessibility of the fresh coal. Five minutes of exposure to oxygen does cause an increase in retention from the 0 second exposure, but the increase is still below the retention in the original fresh coal. After an exposure of 50 minutes, the retention of spin probe VII under both circumstances increases (although to a greater extent in oxidized coal). It would appear that within the first 5 minutes, changes brought about by oxidation inhibit the accessibility of spin probe VII into the coal structure, suggesting an increase in covalent cross-linking. 


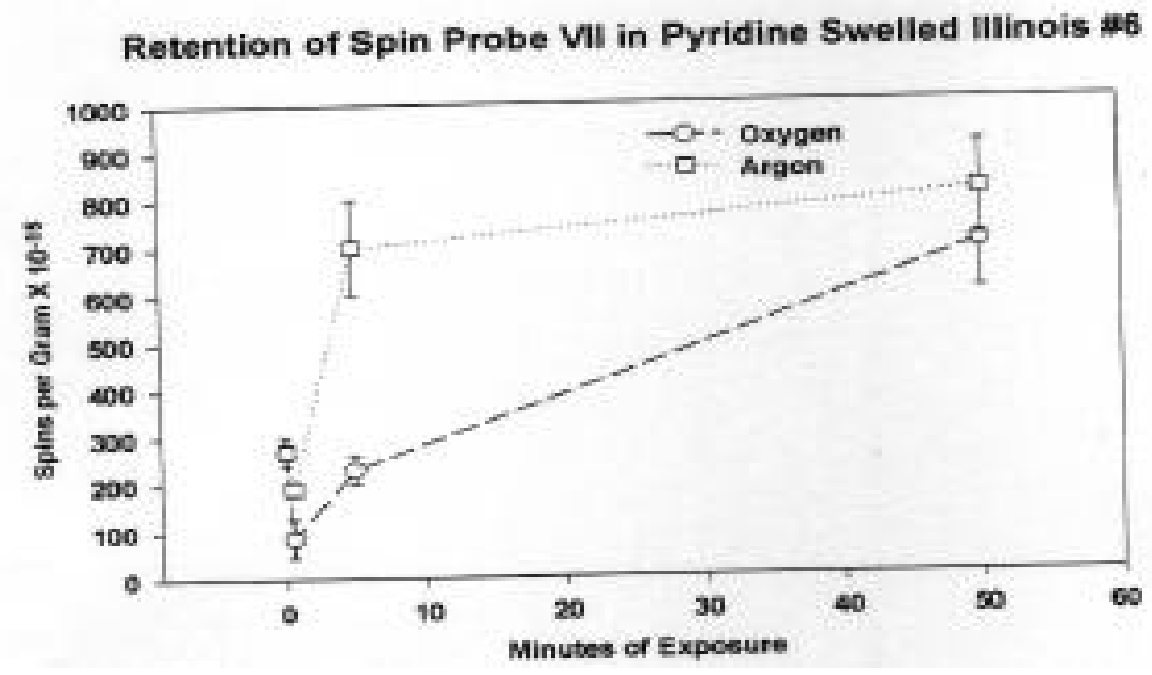

Figure 35. The retention of spin probe VII in pyridine swelled Illinois \#6 coal exposed to both argon $(\square)$ and oxygen $(\mathrm{O})$.

The retention of spin probe VIII in Illinois \#6 coal upon exposure to argon and oxygen for up to 50 minutes is show in Figure 36. Very little change in the structure is observed for 30 seconds of exposure. After 5 minutes of exposure, however, increased retention was observed for both dried and oxidized coal. This is particularly pronounced for oxidized coal. As the exposure time continues beyond five minutes, the retention of spin probe VII in coal exposed to oxygen decreases significantly, while the retention in coal exposed to argon increases. It is clear that oxidation has an effect on the physical structure of the coal which can suppress changes caused by water loss.

Retention of Spin Probe VII in Pyridine Swelled IIIInois \#b

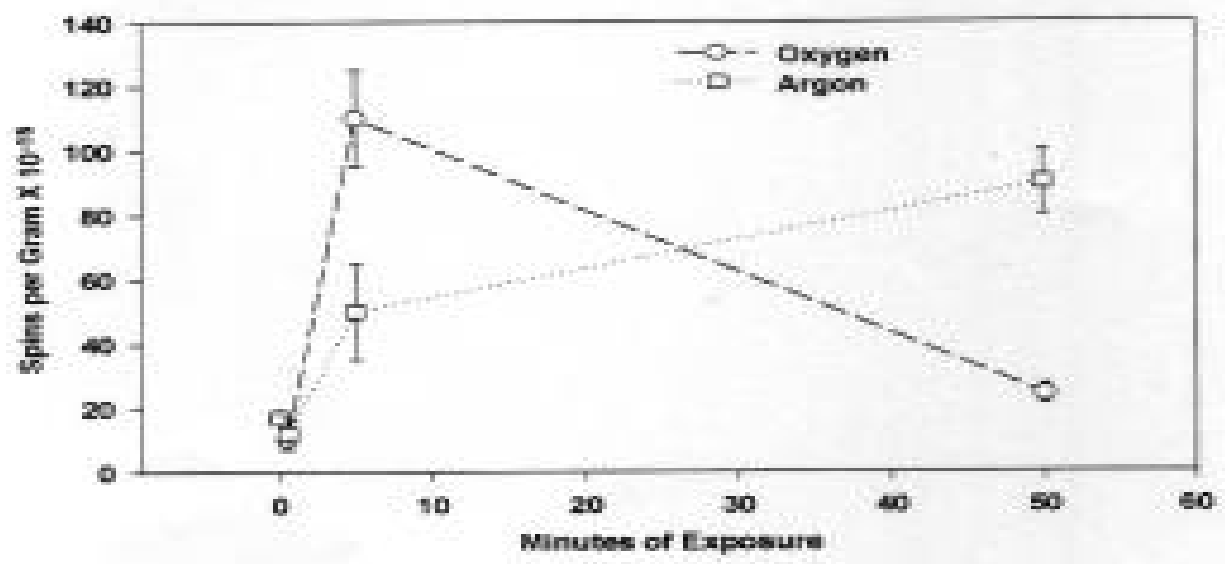

Figure 36. The retention of spin probe VIII in pyridine swelled Illinois \#6 coal exposed to both argon $(\square)$ and oxygen $(\mathrm{O})$.

A comparison of Figures 31 to 33 and 34 to 36 shows that the retention characteristics of spin probe VI closely mirror those of spin probe VIII. This suggests that changes in the physical structure of coal can in influence the retention of spin probe VI as well. Also, since oxidation produces an increase in retention of spin probe VII in coal 
swelled in toluene and a decrease in the retention of spin probe VI, it would seem that oxidation causes an increase in active sights capable of being hydrogen bond donors.

\section{BINARY SWELLING SOLVENT SYSTEMS}

\section{Introduction}

In swelling coals with chlorobenzene, it has been observed by Green and Larsen that when small amounts $(0.35 \mathrm{mmol} / \mathrm{g}$ coal $)$ of pyridine are added to chlorobenzene, the up-take of chlorobenzene by the coal is dramatically increased. ${ }^{61}$ Since the EPR spin probe method has been shown to be very sensitive to changes in the physical and chemical structure of coal during the swelling process, ${ }^{62}$ it was thought that some insight into the interaction between pyridine and the coal structure could be gained by applying this method to a binary swelling solvent solution. Toluene was used as the swelling solvent because it does not significantly swell the coal or disturb any cross-linking, and allows for a more clear study of the interactions between the coal and pyridine.

As it turned out, the results obtained were far from simple to interpret, thus requiring a brief discourse and reinterpretation of some previous work on coal structure. Painter has asserted that hydrogen bonds in coal are short lived and do not make major contributions to the cross-linked structure. ${ }^{63}$ His most recent model assumes that coal is made up of interconnected chains which are solubilized during the swelling process. ${ }^{64}$ These connected chains have segments he refers to as dangling ends, which have mobility limited by the surrounding solubilized chain segments. This view of coal suggests that coal has a dynamic structure during the swelling process. The same conclusion can be reached by studying the result of short term oxidation studies discussed previously. It was determined that structural and chemical changes can be brought about in coal in as little as 30 seconds exposure to argon or oxygen. The primary cause of these changes within 30 seconds was the removal of water during exposure to the dry gas. This indicates that the structure of coal is very sensitive to the moisture content of the environment and undergoes changes at the slightest perturbation, hence showing that coal possesses a dynamic structure.

Although it may be true that hydrogen bond interactions cannot account for significant cross-linking between independent macromolecules in the coal, it must be conceded that hydrogen bonding can establish cross-linking within the macromolecule where hydrogen bond sites have limited maneuverability on proximate chains or segments. Similar hydrogen bonding behavior is observed in inclusion compounds such as calixarenes. It is possible that these hydrogen bond cross-links could block off micropore areas, making them inaccessible to guest molecules until they are disrupted by a swelling solvent. This may be further supported by observations made by Larsen and Wernet. ${ }^{40}$ They did several surface area experiments in which they observed that the surface area of Beulah Zap lignite by $\mathrm{N}_{2}$ or hydrocarbon surface analysis was 30 times less than by $\mathrm{CO}_{2}$ surface analysis. They attributed the large difference to the solubility of $\mathrm{CO}_{2}$ in the coal structure and concluded that an interconnected micropore network did not exist. However, if coal does indeed have a dynamic structure, the hydrogen bond interactions would become stronger as the temperature decreases. This would cause a structural change with decreasing temperature, and account for in accessibility of hydrocarbons and $\mathrm{N}_{2}$ at such low temperatures since these techniques assume that the structure of the macromolecule does not significantly change as the temperature is decreased to $77 \mathrm{~K}$.

This leads to two assumptions necessary to explain the observations made in this study: one, that coal has a dynamic structure and two, that hydrogen bond interactions form a type of cross-linking which inhibits accessibility of guest molecules to the coal 
micropore structure. This research addresses the changes observed when toluene, a weak swelling solvent, is spiked with various amounts of pyridine, a strong swelling solvent.

\section{Results}

Results from this work are described in R. Ding, D. Tucker and L. D. Kispert, "Influence of Binary Swelling Solvent, Prepr. Pap. Am. Chem. Soc. Div. Fuel Chem. 40, 590 (1995).

\section{O-ALKYLATION}

To further examine the swelling behavior in the presence or absence of internal hydrogen bonding using the EPR spin probe technique, known O-alkylation procedures ${ }^{65}$ have been used to remove internal hydrogen bonding in APCS coal samples by derivatizing the hydroxyls. Removing the internal hydrogen bonding prevents the polar spin probe retention. On the other hand, an increase in coal swelling by nonpolar solvents such as toluene was observed. By swelling O-alkylated APCS coals in nonpolar as well as polar solvents using spin probes VII

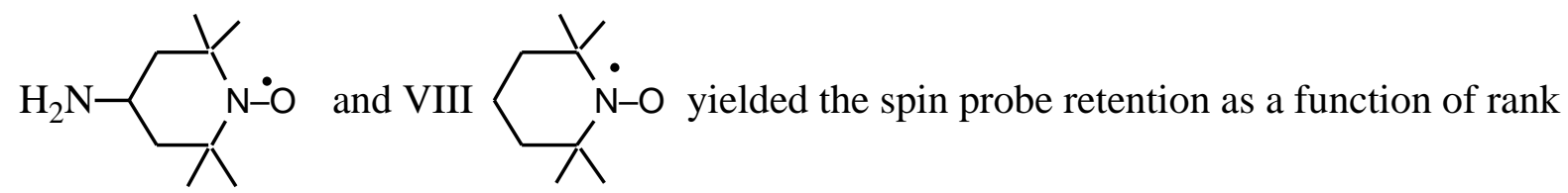

when internal hydrogen bonding due to hydroxyls is removed.

Details have been published on the Influence of Binary Swelling Solvents ${ }^{66}$ and on the Swelling Behavior of O-Alkylated APCS Coal. ${ }^{67}$ It was found that upon O-alkylation, the hydrogen bonding is removed and the microporous structure increases in size. The strong oscillatory variation in spin concentration as a function of percent pyridine in a toluene swelling solvent was eliminated.

\section{INFLUENCE OF SUNLIGHT}

A talk was presented in the coal Liquefaction/Coprocessing Symposium of the National ACS meeting held in Orlando, FL, Aug. 25-29, 1996 entitled "Changes in Molecular Accessibility in APCS Coal Oxidized in the Presence of Sunlight." This study was carried out for Pocahontas \#3, Lewiston-Stockton and Wyodak-Anderson coal in which the oxygen content varied from $2 \%$ to $17 \%$. A paper published 68 on the topic examined the reason that sunlight played an important role in the weathering process. One possible reason is the formation of singlet oxygen by energy transfer from the excited singlet states of the naturally occurring naphthalene and phenoxyl anion radical in the coal to the singlet excited state of oxygen.

This same study was then expanded for Upper Freeport coal ( $8 \%$ oxygen content) to that for Beulah-Zap ( $20 \%$ oxygen content) to that for Illinois \#6 (10\% oxygen content). It has been determined that the microporosity depends on the structure of the coal; coals with similar percent oxygen content do not show the same percent increase in microporosity. In oxygen rich coal, evidence exists for crosslinking as a function of exposure to air and sunlight, with a balance occurring for Illinois \#6, so exposure to sunlight does not cause a change in the penetration of molecular probes.

The uptake of spin probe VII was measured for Illinois \#6 $(\mathrm{C}=80.7 \%$ and $\mathrm{O}=$ $10.1 \%$ ) after the sample had been exposed to air and sunlight and compared to when it was 
alkylated. ${ }^{68}$ A decrease of $17 \%$ in uptake of spin probe VII in air occurred over an eight hour period in the absence of light. A $13 \%$ decrease in the uptake of VII occurred over an eight hour period when the coal sample was exposed to light and air. It was found that very little difference occurred in the up take of VII when the sample was exposed to air in the dark. Apparently a balance occurs between the formation of cross-linking due to hydroperoxide radicals and the increase in hydrogen bonding occurring upon oxidation.

Upon alkylation a more open porous structure was observed as a result of the loss of hydrogen bonding. A $47 \%$ decrease (6.0 to $3.2 \times 10^{18}$ spin per gram) in the uptake of VII occurred in the absence of sunlight. Upon the addition of a trace of pyridine, the uptake of spin probe decreased further ( $52 \%$ decrease), indicating the break-up of the remaining hydrogen bonds.

It was also shown that with time, the micropore structure in Illinois \#6 coal increases in size, causing the loss of spin probe VII upon washing with cyclohexane. This is consistent with the properties of a poor swelling solvent. The decrease in uptake of spin probe VII upon exposure of the Illinois \#6 coal to sunlight confirmed the importance of singlet oxygen in coal oxidation as suggested in an earlier paper. ${ }^{68}$

Upon alkylation, it was previously deduced that cross-linking by the combination of hydroperoxide radicals with radicals existing in the coal occurred for oxygen rich Wyodak-Anderson and also for - Beulah-Zap. As the time of exposure to sunlight increased a decrease in molecular accessibility (decrease in spin probe VII uptake) occurred due to an increase in cross-linking character. However as reported previously for the high rank coal, Upper Freeport, this effect was not present to any significant degree and the increase presence of functional groups as deduced by alkylation and subsequent hydrogen bonding caused an increase in the uptake of polar spin Probe VII. These two mechanism essentially counter-balanced each other for Lewiston-Stockton. In Illinois \#6, a more open structure occurs upon alkylation. These studies confirm the previous mechanism that in high rank coal, the presence of singlet oxygen leads to an increase in hydrogen bonding and a subsequent increase in molecular accessibility.

\section{CONCLUSIONS}

The EPR spin probe method has been shown to be very sensitive to both dehydration and oxidation of coals during the weathering proccess. The structural of the lower ranked coals upon weathering was determined to be primarily due to the loss of moisture and volatile components from the coal. The medium ranked coals were observed to possess enough cross-linking to resist structural collapse so that the initial loss of water and volatile components simply provided additional active sites capable of interaction with polar spin probes. The removal of moisture was also shown to have a strong effect on high-rank coals swelled in pyridine.

The oxidation of vacuum dried coals in pure oxygen has provided valuable insight into the oxidation process in coal. It was determined that the observed changes in spin probe retention during oxidation could be explained in terms of the autoxidation process observed in other polymeric systems. High-rank coals, which do not exhibit large changes in spin probe retention upon vacuum drying, exhibited changes in spin probe retention upon oxidation which spanned three orders of magnitude. The changes in spin probe retention also provided evidence that increased covalent cross-linking can occur during the initial stages of oxidation. The changes in spin probe VI, VII and VIII retention character were summarized for the oxidation process. 
In general, the initial stages of oxidation result in the formation of hydroperoxides or other polar functional groups which result in the formation of increased hydrogen bonding. This is followed by a significant increase in covalent cross-linking. Further oxidation results in degradation of covalent cross-linking and increased formation of polar functional groups. Fluctuations in the concentrations of spin probes VI and VII are observed as $\mathrm{C}-\mathrm{OH}$ is converted to $\mathrm{C}=\mathrm{O}$ and $\mathrm{COOH}$. In some of the APCS coals, formation of $\mathrm{CO}_{2}$ is evidenced by an increase in non-polar micropores over polar micropores of small, spherical size and shape.

\section{Short Term Oxidation}

It has been clearly shown that significant structural changes can occur in coal in as little as 30 seconds of exposure to a dry gas environment. Likewise, oxidational changes were observed to take place in as little as five minutes of exposure to an oxygen environment. Exposure to oxygen for even small periods of time have resulted in increased covalent cross-linking, which inhibits the ability of pyridine to open the coal structure. These results may provide some insight into some of the problems with obtaining consistent data inherent to coal research.

\section{Binary Swelling Solvents}

Increased inclusion of guest molecules into the macromolecular structure of coal can be accomplished by spiking toluene with small amounts of a strong swelling solvent like pyridine. The best amount to be added to achieve maximum retention of guest molecules varies with rank. The EPR Spin Probe technique is sensitive to changes in swelling characteristics of coal in pyridine/toluene binary swelling solvent systems. Changes can be observed at pyridine concentrations as low as 100ppm. Dramatic oscillations in spin probe retention are observed as the amount of pyridine in the swelling solvent mixture is increased, especially in amounts representing less than $1 \%$ pyridine. This provides some indication that several types of hydrogen bonded cross-linking are present.

\section{RECOMMENDATIONS: FUTURE WORK}

\section{Humidity}

The relationship of moisture to coal structure should be studied in more detail. The results of the previous weathering experiments have indicated that there is an intimate relationship between water and coal structure. This relationship could be further examined by placing the fresh coals under an inert environment with a controlled humidity. The samples could then be allowed to come to an equilibrium under various humidities so that accessibility could be determined as a function of environmental moisture content. The effect of moisture on the oxidation process could likewise be studied in more detail by using oxygen or air instead of an inert gas.

\section{Oxidation}

The results of the oxidation studies suggest that the oxidation of coals proceeds by the autoxidation process and that this process causes increased covalent cross-linking in the coal structure. If this is the case, insight into this process could be gained by examining the free radical content of the coal as oxidation occurs. Oxidation studies could be done in coordination with an EPR study of unswelled aliquots of the oxidized coal to see how the 
radical content of the coal is affected during oxidation. Additionally, IR studies could be done on aliquots of the same samples to monitor the functionalities during oxidation to substantiate observations of polar spin probe retention.

\section{Short Term Oxidation}

The initial study of short term oxidation has shown that significant changes can occur in the Illinois \#6 coal structure in as little as 30 seconds. Similar oxidation studies could provide in formation about the other APCS coals so that this effect could be studied as a function of rank Since it was determined that the largest effects were observed in under five minutes, short term oxidation studies should be carried out focusing on time periods under five minutes.

\section{Binary Swelling Solvent Systems}

Since small amounts of pyridine can be used to change the accessible nature of coal during swelling, it would be valuable to examine the effects of other binary systems, especially one spiked

with water, since water is strongly polar and is shown to have such an intimate relationship with the coal structure. It might also be valuable to examine the effects of binary swelling solvent on medium and high ranked coals. Also, since the more significant changes in spin probe retention occur at concentrations less than $1 \%$ for binary systems used on the lower ranked coals, subsequent studies should focus more on these concentrations.

\section{REFERENCES}

1. Larsen, J. W.; Green, T. K.; Kovak, J. J. Org. Chem. 1985, 50, 4729.

2. Vorres, K. S. Energy and Fuels 1990, 4, 420.

3. Spears, D. R.; Kispert, L. D.; Piekara-Sady, L. Fuel 1992, 71, 1003.

4. Sady, W.; Kispert, L. D.; Spears, D. R. Prepr. Pap. Am. Chem. Soc., Div. Fuel 1992, 37, 1151.

5. Spears, D. R.; Sady, W.; Kispert, L. D. Prepr. Pap. Am. Chem. Soc., Div. Fuel 1991, 36, 1277.

6. Goslar, J.; Kispert, L. D. Energy and Fuels 1989, 3, 589.

7. Tucker, D.; Kispert, L. D. Prepr. Pap. Am. Chem. Soc., Div. Fuel Chem. 1993 38, 1330 .

8. Sady, W.; Tucker, D.; Kispert, L. D.; Spears, D. R. Prepr. Pap. Am. Chem. Soc., Div. Fuel Chem. 1993, 38, 1323.

9. Spears, D. R.; Sady, W.; Tucker, D.; Kispert, L. D. Energy and Fuels 1993, 7, 1001.

10. Cody, G. D.; Larsen, J. W.; Siskin, M. Energy and Fuels 1988, 2, 340.

11. Silbernagel, B.G.; Ebert L.B.; Scholsberg, R.H.; and Long, R.B. Adv. Chem. Ser., 240, 1979 
12. Speards, R.; Goslar, J.; Kispert L.D. "Magnetic Resonance of Carbonaceous Solids"; Botto R.E.; Sanada, Y., Eds.; Advances in Chemistry 229; American Chemical Society: 240, Washington, DC, 1993, p. 467.

13. Spears, D.R.; Kispert L.D.; Piekara-Sady, L. Prepr. Pap. Am. Chem. Soc., Div. Fuel. Chem. 1991 36, 29.

14. Spears, D.R. Ph. .D. Dissertation, University of Alabama, Tuscaloosa, 1991.

15. Spears, D.R.; Kispert, L.D.; Sady, W. Fuel 1993 72, 1225.

16. Schafer, H.N.S. Fuel, 1970, 49, 17.

17. Diamond, R. Acta Crustallogr. 1957 10, 359.

18. Larsen, J.W.; Mohammadi, M. Energy \& Fuels 1990, 4, 107.

19. Nishioka, M.; Larsen, J.W. Energy \& Fuels 1990, 4, 100.

20. Ruberto, R.G.; Cronauer, D.C.; Jewell, P.M.; Seshadri, K.S. Fuel 1977, 56, 25.

21. Ouichi, K.; Brooks, J.D. Fuel 1967, 46, 367.

22. Ouichi, K.; Imuta, K. Fuel 1973, 52, 171.

23. Ignasipak, B.; Gawlak, M.; Fuel 1977, 56, 216.

24. Green,T.; Kovac, J.; Brenner, D.; Larsen, J.W. In Coal Structure; Meryers, R.A.. Ed.; Academic: New York, 1982.

25. Fisher, C.H.; Eisner, A.; Ind. Eng. Chem. 1937, 29, 1371.

26. Barr-Howell, B.D.; Peppas, N. J. Appl. Polymer Sci. 1986, 31, 39.

27. Milewskaduda, J. Fuel, 1987, 66, 1570.

28. Painter, P.C.; Park, Y.; Coleman, M.M. Energy \& Fuels 1988, 2, 693.

29. Hall, P.J.; Marsh, H.; Thomas, K.M. Fuel 1988, 67, 863.

30. Berkowitz, N. "An Introduction to Coal Technology," Academic: New York, 1975.

31. Sani, A.K.; Song, C.; Schobert, H.H. Prepr. Pap. Am. Chem. Soc., Div. Fuel Chem. 1993, 38, 593.

32. Sani, A.K.; Song, C.; Schobert, H.H. Prepr. Pap. Am. Chem. Soc., Div. Fuel Chem. 1993, 38, 600.

33. Farmer, E.H. Trans. Faraday Soc. 1942, 38, 356.

34. Stewart, R. "Oxidation Mechanisms," W.A. Benjamin, Inc., New York, 1964 p.15. 
35. Lundberg, W.O. "Autoxidation and Antioxidants," Interscience Publishers, New York, 1961.

36. Rieche, A.; Koch, K. XIV, Ber. Deut. Chem. Ges. 1942, 75, 1016.

37. Markova, K.; Radev, G.; Kostova, N. Khim. Tverd. Topl. (Moscow), 1992, 3, 20.

38. Itoh, M.; Demurs, A.; Oda, H.; Yokokawa, C. Coal Sci. Technol., $11^{\text {th }}$ (Int. Conf. Coal Sci., 1987, 527.

39. Larsen, J. W.; Wernett, P. C. Energy and Fuels 1988, 2(5), 719.

40. Larsen, J. W.; Wernett, P. C. Prepr. Pap. Am. Chem. Soc., Div. Fuel Chem. 1992, $37(2), 849$.

41. Kalliat, M.; Kwak, C. Y.; Schmidt, P. W. ACS Symp. Ser., 169 (New Approaches Coal Chem. 1981, 3.

42. Setek, M.; Wagonfeld, H. K.; Stacy, W. O.; Kiss, L. T. Fuel, 1983, 62(4), 480.

43. Gethner, J. S. J. Appl. Phys., 1986, 59(4), 1068.

44. Tricker, M. J.; Grint, A.; Audley, G. J.; Church, S. M.; Rainy, V. S.; Wright, C. J. Fuel 1983, 62(9), 1092.

45. Kaiser, H.; Gethner, J. S. Proc.-Inter. Conf. Coal Sci. 1983, 300.

46. Gethner, J. S. Prepr. Pap. Am. Chem. Soc., Div. Fuel Chem. 1987, 32(1), 239.

47. Nemmers, S.; Horne, D. K.; Bale, H. D. J. Appl. Phys. 1990, 68(7), 3178.

48. Cody, G. D.; Davis, A. Energy and Fuels 1991, 5(6), 776.

49. Cody, G. D.; Larsen, J. W.; Siskin, M. Energy and Fuels 1988, 2, 340.

50. Glaves, C. L.; Davis, P. J.; Gallegos, D. P.; Smith D. M. Energy and Fuels 1988, $2(5), 662$.

51. Wernett, P. C.;Larsen, J. W.;Yamada, O.; Yue, H. J. Energy and Fuels 1990, 4(4), 412.

52. Tsiao, C.; Botto, R. E. Energy and Fuels 1991, 5(1), 87.

53. Wuu, S. K.; Kispert, L. D. Fuel 1985, 64, 1681.

54. Goslar, J.; Cooray, L. S.; Kispert, L. D. Fuel 1989, 68, 1402.

55. Goslar, J.; Kispert, L. D. Fuel 1990, 69, 564.

56. Tucker, D.; Kispert, L. D. Prepr. Pap. Am. Chem. Soc., Div. Fuel Chem. 1993 38, 1335.

57. Cooray, L.S. M.S. Thesis, University of Alabama, 1988. 
58. Hudlicky, M. "Oxidations in Organic Chemistry," American Chemical Society, Washington D.C. 1990.

59. Yongseung, Y.; Suuberg, E.M. Prepr. Pap. Am. Chem. Soc., Div. Fuel Chem. 1992, 37, 1184.

60. Graff, R.A.; Brandes, S.D. Energy and Fuels 1987, 84.

61. Green, T.K. and Larsen, J.W. Fuel 1984 63, 1538.

62. Sady, W.; Tucker, D.; Kispert, L.D. and Spears, D.R. Prepr. Pap. Am. Chem. Soc., Div. Fuel Chem. 1993, 38, 1323.

63. Painter, P. Am. Chem. Soc., Div. Fuel Chem. Symp. Macrom. Str. Coal. August 22 - 26, 1993, Chicago, IL.

64. Painter, P. Prepr. Pap. Am. Chem. Soc., Div. Fuel Chem. 1993.

65. R. Liotta, K. Rose and E. Hippo, J. Org. Chem. 1981 46, 277.

66. R. Ding, D. Tucker and L. D. Kispert, Prepr. Pap. Am. Chem. Soc., Div. Fuel Chem. 1995, 40, 590 (1995).

67. R. Ding, D. Tucker and L. D. Kispert, Prepr. Pap. Am. Chem. Soc., Div. Fuel Chem. 1995, 40, 425 (1995).

68. R. Ding, L. D. Kispert and A. S. Jeevarajan, Prepr. Pap. Am. Chem. Soc. Div, Fuel Chem. 1996, 41, 1094. 


\section{APPENDIX - List of Published Papers}

1. L. D. Kispert, D. Tucker, W. Sady, Prepr. Pap. Am. Chem. Soc., Div. Fuel. Chem. $1994,39,54$.

2. R. Ding, D. Tucker and L. D. Kispert, Prepr. Pap. Am. Chem. Soc., Div. Fuel. Chem. 1995, 40, 590.

3. R. Ding, D. Tucker and L. D. Kispert, Prepr. Pap. Am. Chem. Soc., Div. Fuel Chem. 1995, 40, 425.

4. R. Ding, L. D. Kispert and A. S. Jeevarajan, Prepr. Pap. Am. Chem. Soc. Div., Fuel Chem. 1996, 41, 1094. 